Suporting Information for

\title{
Dragmacidin E Synthesis Studies. Preparation of a Model Cycloheptannelated Indole Fragment.
}

\author{
Ken S. Feldman* and Paiboon Ngernmeesri \\ Department of Chemistry, the Pennsylvania State University, University Park, \\ Pennsylvania 16802 USA
}

\begin{tabular}{|c|c|c|c|}
\hline General Experimental & S2 & ${ }^{1} \mathrm{H}$ NMR 11 & S30 \\
\hline 8 & S2 & 13 C NMR 11 & S31 \\
\hline 9 & S4 & ${ }^{1} \mathrm{H}$ NMR SM3 & S32 \\
\hline SM1 & S5 & ${ }^{13}$ C NMR SM3 & S33 \\
\hline SM2 & S6 & ${ }^{1}$ H NMR 12 & S34 \\
\hline 11 & $\mathrm{~S} 7, \mathrm{~S} 8$ & ${ }^{13}$ C NMR 12 & S35 \\
\hline SM3 & S7, S9 & ${ }^{1} \mathrm{H}$ NMR SM4 & S36 \\
\hline 12 & S9 & ${ }^{13}$ C NMR SM4 & S37 \\
\hline SM4 & $\mathrm{S} 10$ & ${ }^{1}$ H NMR SM5 & S38 \\
\hline SM5 & S11 & ${ }^{13}$ C NMR SM5 & S39 \\
\hline 13 & S12 & ${ }^{1}$ H NMR 13 & S40 \\
\hline 15 & S13 & ${ }^{13}$ C NMR 13 & S41 \\
\hline 16 & S14 & ${ }^{1} \mathrm{H}$ NMR $c i s-\mathbf{1 5}$ & S42 \\
\hline SM6 & S15 & ${ }^{13} \mathrm{C}$ NMR cis-15 & S43 \\
\hline 17 & S16 & 'H NMR trans-15 & S44 \\
\hline SM7 & S17 & 13. C NMR trans -15 & S45 \\
\hline 18 & S18 & ${ }^{1} \mathrm{H}$ NMR 16 & S46 \\
\hline${ }^{1} \mathrm{H}$ NMR 8 & S20 & ${ }^{13} \mathrm{C}$ NMR 16 & S47 \\
\hline${ }^{13}$ C NMR 8 & S21 & ${ }^{1}$ H NMR SM6 & S48 \\
\hline '11 H NMR 9 & S22 & ${ }^{13} \mathrm{C}$ NMR SM6 & S49 \\
\hline 13 C NMR 9 & S23 & ${ }^{1} \mathrm{H}$ NMR 17 & S50 \\
\hline${ }^{1}$ H NMR SM1 & S24 & 13 C NMR 17 & S51 \\
\hline${ }^{13}$ C NMR SM1 & S25 & ${ }^{1} \mathrm{H}$ NMR SM7 & S52 \\
\hline${ }^{1} \mathrm{H}$ NMR trans-SM2 & S26 & ${ }^{13} \mathrm{C}$ NMR SM7 & S53 \\
\hline${ }^{13} \mathrm{C}$ NMR trans-SM2 & S27 & ${ }^{1}$ H NMR 18 & S54 \\
\hline 'H NMR cis-SM2 & S28 & ${ }^{13}$ C NMR 18 & S55 \\
\hline${ }^{13}$ C NMR cis-SM2 & S29 & MM calc's of 15 -imine & \\
\hline
\end{tabular}


General Experimental. Unless stated otherwise, moisture and oxygen sensitive reactions were carried out in flame-dried glassware under a nitrogen or argon atmosphere using anhydrous, deoxygenated solvents. Tetrahydrofuran was dried by passage through an activated alumina column under a nitrogen atmosphere or distillation from sodium benzophenone ketyl under an argon atmosphere. Dichloromethane and acetonitrile were dried by passage through an activated alumina column under a nitrogen atmosphere or distillation from calcium hydride under an argon atmosphere. HPLC grade acetonitrile was also used as received for large scale photochemical reactions and it did not significantly lower the yield of the product. Methanol was dried by distillation from calcium hydride under a nitrogen or argon atmosphere. Absolute ethanol was used as received. All other commercially obtained reagents were used as received. Microwave reactions were performed in the CEM Focused Microwave ${ }^{\mathrm{TM}}$ Synthesis System, Model Discover. Flash chromatography was performed on $32-63 \mu \mathrm{m}$ silica gel. The characterization data were obtained on the solid material that resulted from evaporation of the chromatography solvents used for purification. Melting points were taken with a Melt-Temp apparatus and are uncorrected. Chemical shifts of ${ }^{1} \mathrm{H}$ NMR spectra are reported relative to $\mathrm{Me}_{4} \mathrm{Si}(\delta 0.00)$ or DMSO $(\delta 2.49)$ if the former was absent. ${ }^{13} \mathrm{C}$ NMR spectra are reported relative to $\mathrm{Me}_{4} \mathrm{Si}(\delta 0.0)$, or $\mathrm{CDCl}_{3}(\delta 77.0)$ or DMSO $(\delta 39.5)$ if the former was absent.
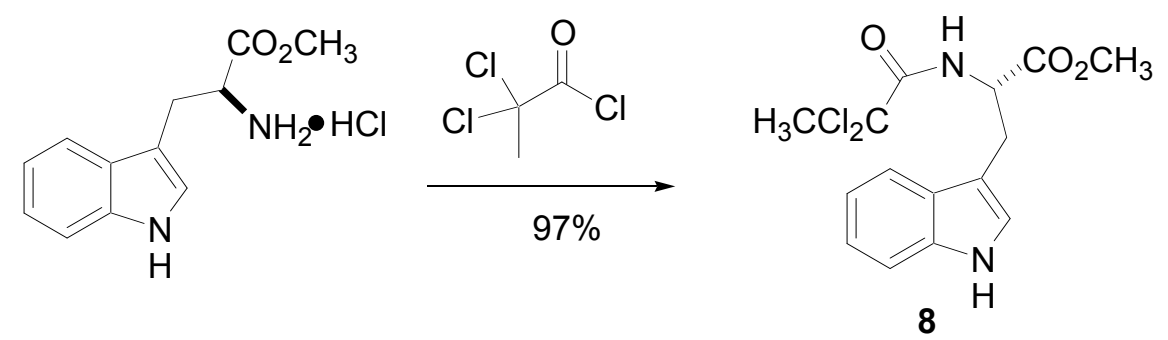
Methyl 2-(2,2-Dichloropropionylamino)-3-(1H-indol-3-yl) Propionate (8). To a solution of 2,2-dichloropropionic acid $(90 \%, 6.7 \mathrm{~mL}, 59 \mathrm{mmol})$ in $\mathrm{CH}_{2} \mathrm{Cl}_{2}(50 \mathrm{~mL})$ was added 2 drops of DMF followed by oxalyl chloride $(10.0 \mathrm{~mL}, 119 \mathrm{mmol})$. The reaction mixture was stirred for $2.5 \mathrm{~h}$ until bubbling stopped. The resulting yellow acid chloride solution was concentrated under reduced pressure and then redissolved in $\mathrm{CH}_{2} \mathrm{Cl}_{2}(40$ $\mathrm{mL}$ ). The acid chloride solution was cannulated to an ice-cooled solution of Ltryptophan methyl ester hydrochloride $(10.01 \mathrm{~g}, 39 \mathrm{mmol})$ and dimethylaminopyridine (10.05 g, $82 \mathrm{mmol})$ in $\mathrm{CH}_{2} \mathrm{Cl}_{2}(60 \mathrm{~mL})$. The resulting red solution was stirred in an ice bath for $3 \mathrm{~h}$ and then at room temperature for $12 \mathrm{~h}$. The reaction mixture was poured into ice water $(50 \mathrm{~mL})$ and the organic layer was separated. The aqueous layer was extracted with $\mathrm{CH}_{2} \mathrm{Cl}_{2}(2 \times 50 \mathrm{~mL})$. The combined organic layers were dried over anhydrous $\mathrm{Na}_{2} \mathrm{SO}_{4}$ and concentrated under reduced pressure. The crude residue was purified by flash chromatography on silica gel (2:1 hexanes/EtOAc) to afford the dichloroamide $\mathbf{8}$ as a light brown solid (13.13 g, 97\%). mp 143-144 ${ }^{\circ} \mathrm{C}$; $[\alpha]^{20}{ }_{\mathrm{D}}+51^{\circ}\left(\mathrm{c} 1.00, \mathrm{CHCl}_{3}\right)$; IR (film) 3368, 3315, 1728, $1678 \mathrm{~cm}^{-1} ;{ }^{1} \mathrm{H}$ NMR (300 MHz, $\left.\mathrm{CDCl}_{3}\right) \delta 8.26$ (br s, 1H), 7.55 $(\mathrm{d}, J=7.8 \mathrm{~Hz}, 1 \mathrm{H}), 7.34(\mathrm{~d}, J=8.0 \mathrm{~Hz}, 1 \mathrm{H}), 7.29$ (br s, $1 \mathrm{H}), 7.19$ (ddd, $J=8.0,7.0,1.3$ $\mathrm{Hz}, 1 \mathrm{H}), 7.11(\mathrm{ddd}, J=7.8,7.0,1.1 \mathrm{~Hz}, 1 \mathrm{H}), 7.02(\mathrm{~d}, J=2.1 \mathrm{~Hz}, 1 \mathrm{H}), 4.86(\mathrm{ddd}, \mathrm{J}=7.7$, 5.3, $5.3 \mathrm{~Hz}, 1 \mathrm{H}), 3.69(\mathrm{~s}, 3 \mathrm{H}), 3.42(\mathrm{dd}, J=18.5,5.2 \mathrm{~Hz}, 1 \mathrm{H}), 3.37(\mathrm{dd}, J=18.9,5.1 \mathrm{~Hz}$, 1H), $2.25(\mathrm{~s}, 3 \mathrm{H}) ;{ }^{13} \mathrm{C} \mathrm{NMR}\left(300 \mathrm{MHz}, \mathrm{CDCl}_{3}\right) \delta 171.4,165.9,136.1,127.3,123.0$, 122.3, 119.7, 118.5, 111.3, 109.2, 82.1, 53.8, 52.6, 33.9, 27.2; LRMS(ESI) $m / z$ (relative intensity) $365.0\left(100 \%, \mathrm{M}+\mathrm{Na}^{+}\right)$; HRMS (ESI) $\mathrm{m} / z$ calcd for $\left[\mathrm{C}_{15} \mathrm{H}_{16} \mathrm{~N}_{2} \mathrm{O}_{3} \mathrm{Cl}_{2} \mathrm{Na}\right]^{+}$: 365.0436 , found 365.0426 . 

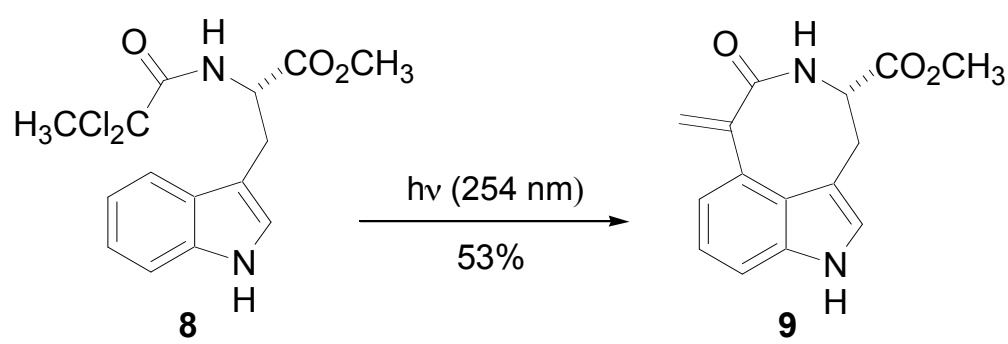

\section{Methyl 7-Methylene-6-oxo-1,3,4,5,6,7-hexahydro-azocino[4,5,6-cd]indole-4}

Carboxylate (9). A solution of dichloroamide 8 (150 mg, $0.44 \mathrm{mmol})$ in $\mathrm{CH}_{3} \mathrm{CN}(87.5$ $\mathrm{mL}$ ) in a quartz vessel was purged with dry argon for $30 \mathrm{~min}$. This solution was irradiated at $254 \mathrm{~nm}$ in a Rayonet photochemical reactor for $3 \mathrm{~h}$. The resulting light brown solution was concentrated under reduced pressure. The crude residue was purified by flash chromatography on silica gel (1:3 hexanes/EtOAc) to afford the bridged indole 9 as a yellow solid $(63 \mathrm{mg}, 53 \%) . \mathrm{mp} 194-195{ }^{\circ} \mathrm{C} ;[\alpha]^{20}{ }_{\mathrm{D}}-347^{\circ}\left(c 0.60, \mathrm{CHCl}_{3}\right)$; IR (film) 3305, 1741, $1653 \mathrm{~cm}^{-1} ;{ }^{1} \mathrm{H}$ NMR $\left(300 \mathrm{MHz}, \mathrm{CDCl}_{3}\right) \delta 8.42$ (br s, $\left.1 \mathrm{H}\right), 7.35$ (ddd, $J=8.2$, 4.5, $3.5 \mathrm{~Hz}, 1 \mathrm{H}), 7.20-7.15(\mathrm{~m}, 2 \mathrm{H}), 7.07(\mathrm{dd}, J=1.3,1.2 \mathrm{~Hz}, 1 \mathrm{H}), 6.33(\mathrm{~d}, J=9 \mathrm{~Hz}$, 1H), $5.60(\mathrm{~d}, J=0.9 \mathrm{~Hz}, 1 \mathrm{H}), 5.50(\mathrm{~d}, J=0.7 \mathrm{~Hz}, 1 \mathrm{H}), 5.07$ (ddd, $J=11.8,9.0,2.8 \mathrm{~Hz}$, 1H), 3.84 (s, 3H), 3.56 (ddd, $J=16.3,3.0,0.9 \mathrm{~Hz}, 1 \mathrm{H}), 3.29$ (ddd, $J=16.3,11.8,0.9 \mathrm{~Hz}$, $1 \mathrm{H}) ;{ }^{13} \mathrm{C}$ NMR $\left(300 \mathrm{MHz}, \mathrm{CDCl}_{3}\right) \delta 172.7,171.7,147.8,136.6,129.8,124.2,122.9$, 122.0, 121.7, 116.6, 112.2, 110.4, 55.7, 52.9, 33.1; LRMS(ESI) $m / z$ (relative intensity) $293.1\left(97 \%, \mathrm{M}+\mathrm{Na}^{+}\right)$; HRMS (ESI) $m / z$ calcd for $\left[\mathrm{C}_{15} \mathrm{H}_{14} \mathrm{~N}_{2} \mathrm{O}_{3} \mathrm{Na}\right]^{+}: 293.0896$, found 293.0902. Anal. Calcd for $\mathrm{C}_{15} \mathrm{H}_{14} \mathrm{~N}_{2} \mathrm{O}_{3}$ : C, 66.66; H, 5.22; N, 10.36. Found: $\mathrm{C}, 66.75 ; \mathrm{H}$, $5.32 ; \mathrm{N}, 10.20$. 


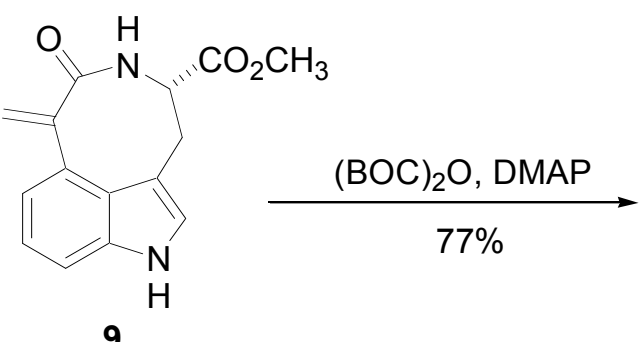

9

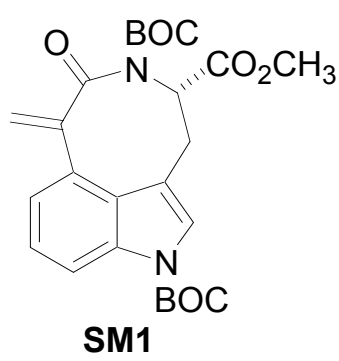

SM1

\section{1,5-Di-tert-butyl 4-Methyl 7-Methylene-6-oxo-3,4,6,7-tetrahydro-azocino[4,5,}

6-cd] indole 1,4,5-Tricarboxylate (SM1). A solution of di-tert-butyldicarbonate (1.13 g,

$5.18 \mathrm{mmol})$ in $\mathrm{CH}_{3} \mathrm{CN}(6 \mathrm{~mL})$ was cannulated into a suspension of indole $9(335 \mathrm{mg}$, $1.24 \mathrm{mmol}$ ) and 4-dimethylaminopyridine (34 $\mathrm{mg}, 0.28 \mathrm{mmol})$ in $\mathrm{CH}_{3} \mathrm{CN}(6 \mathrm{~mL})$. After the addition was complete, the indole 9 slowly became soluble and it all went into solution in less than $10 \mathrm{~min}$. The reaction mixture was stirred at room temperature for 2 h. The resulting dark brown solution was poured into ice water $(20 \mathrm{~mL})$ and extracted with ether $(3 \times 20 \mathrm{~mL})$. The organic extracts were combined, washed with brine solution (20 mL), dried over anhydrous $\mathrm{Na}_{2} \mathrm{SO}_{4}$, and concentrated under reduced pressure. The crude residue was purified by flash chromatography on silica gel (3:2 hexanes/ether) to afford the desired protected product as a white solid (446 mg, 77\%). mp $118-120{ }^{\circ} \mathrm{C}$; $[\alpha]_{\mathrm{D}}^{20}+61^{\circ}\left(c\right.$ 1.00, $\left.\mathrm{CHCl}_{3}\right)$; IR (film) 1734, $1693 \mathrm{~cm}^{-1} ;{ }^{1} \mathrm{H}$ NMR $\left(300 \mathrm{MHz}, \mathrm{CDCl}_{3}\right) \delta$ $8.30(\mathrm{~d}, J=6.5 \mathrm{~Hz}, 1 \mathrm{H}), 7.53(\mathrm{~s}, 1 \mathrm{H}), 7.38-7.29(\mathrm{~m}, 2 \mathrm{H}) 6.47(\mathrm{~d}, J=1.0 \mathrm{~Hz}, 1 \mathrm{H}), 6.05(\mathrm{~d}$, $J=1.0 \mathrm{~Hz}, 1 \mathrm{H}), 4.77(\mathrm{dd}, J=12.7,4.3 \mathrm{~Hz}, 1 \mathrm{H}), 3.85(\mathrm{~s}, 3 \mathrm{H}), 3.30(\mathrm{dd}, J=15.3,4.3 \mathrm{~Hz}$, 1H), 3.11 (ddd, $J=15.2,12.8,1.2 \mathrm{~Hz}, 1 \mathrm{H}), 1.67(\mathrm{~s}, 9 \mathrm{H}), 1.08(\mathrm{~s}, 9 \mathrm{H}) ;{ }^{13} \mathrm{C}$ NMR $(300$ $\left.\mathrm{MHz}, \mathrm{CDCl}_{3}\right) \delta 174.3,170.7,151.4,149.0,144.6,136.6,129.5,129.4,127.2,125.7$, 124.3, 124.0, 116.1, 115.7, 83.9, 82.9, 57.4, 52.6, 28.1, 27.4, 26.7; LRMS(ESI) $\mathrm{m} / \mathrm{z}$ (relative intensity) $493.2\left(100 \%, \mathrm{M}+\mathrm{Na}^{+}\right)$; HRMS (ESI) $\mathrm{m} / z$ calcd for $\left[\mathrm{C}_{25} \mathrm{H}_{30} \mathrm{~N}_{2} \mathrm{O}_{7} \mathrm{Na}\right]^{+}$: 
493.1951, found 493.1944. Anal. Calcd for $\mathrm{C}_{25} \mathrm{H}_{30} \mathrm{~N}_{2} \mathrm{O}_{7}$ : C, 63.82; H, 6.43; N, 5.95. Found: C, 63.83; H, 6.47; N, 5.83 .

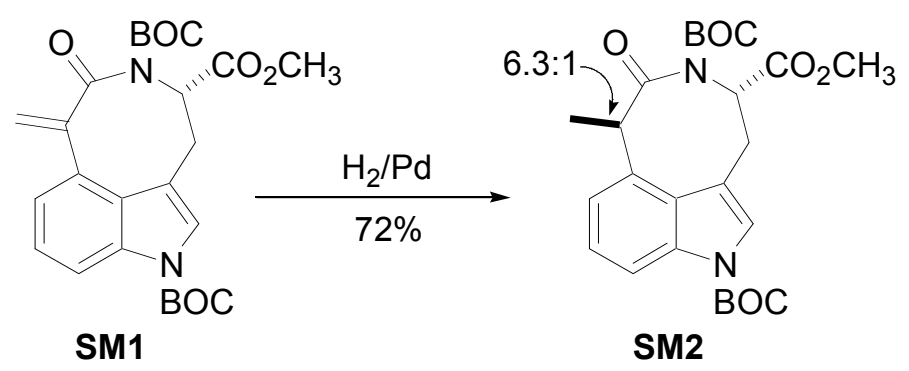

\section{1,5-Di-tert-butyl 4-Methyl 7-Methyl-6-oxo-3,4,6,7-tetrahydro-azocino[4,5,6-}

cd]indole 1,4,5-Tricarboxylate (SM2). To a solution of indole SM1 (483 mg, 1.03 mmol) in $\mathrm{MeOH}(15 \mathrm{~mL})$ was added $10 \% \mathrm{w} / \mathrm{w} \mathrm{Pd} / \mathrm{C}(22 \mathrm{mg}, 0.02 \mathrm{mmol})$. The reaction mixture was stirred under a $\mathrm{H}_{2}$ atmosphere (1atm) for 2 days. The resulting black suspension was filtered through a Celite pad and the filtrate was concentrated under reduced pressure. The crude residue was purified by flash chromatography on silica gel (1:1 hexanes/ether) to afford both the trans-methyl imide SM2 as a white solid (309 mg, 62\%) and the cis-methyl imide SM2 as a white solid (49 mg, 10\%). Major product: mp 167-168 ${ }^{\circ} \mathrm{C} ;[\alpha]^{20}{ }_{\mathrm{D}}-296^{\circ}\left(\mathrm{c} 1.00, \mathrm{CHCl}_{3}\right)$; IR (film) $1734 \mathrm{~cm}^{-1} ;{ }^{1} \mathrm{H} \mathrm{NMR}(300 \mathrm{MHz}$, $\left.\mathrm{CDCl}_{3}\right) \delta 8.07(\mathrm{~d}, J=8.1 \mathrm{~Hz}, 1 \mathrm{H}), 7.44(\mathrm{~s}, 1 \mathrm{H}), 7.29(\mathrm{t}, J=7.9 \mathrm{~Hz}, 1 \mathrm{H}), 7.17(\mathrm{~d}, J=7.6$ Hz, 1H), 5.59 (t, $J=9.5 \mathrm{~Hz}, 1 \mathrm{H}), 4.48(\mathrm{q}, J=6.6 \mathrm{~Hz}, 1 \mathrm{H}), 3.84(\mathrm{~s}, 3 \mathrm{H}), 3.57$ (d, $J=9.5$ $\mathrm{Hz}, 2 \mathrm{H}), 1.67$ (d, $J=6.8 \mathrm{~Hz}, 3 \mathrm{H}), 1.65$ (s, 9H), 1.00 (s, 9H); ${ }^{13} \mathrm{C}$ NMR (300 MHz, $\left.\mathrm{CDCl}_{3}\right) \delta 175.9,172.0,153.3,149.3,135.6,130.9,129.0,125.0,124.5,119.6,114.9$, $114.4,83.8,82.3,56.0,52.9,43.9,29.6,28.1,27.2,12.2$; LRMS(ESI) $\mathrm{m} / z$ (relative intensity) $495.1\left(30 \%, \mathrm{M}+\mathrm{Na}^{+}\right)$; HRMS (ESI) $m / z$ calcd for $\left[\mathrm{C}_{25} \mathrm{H}_{32} \mathrm{~N}_{2} \mathrm{O}_{7} \mathrm{Na}\right]^{+}: 495.2107$, found 495.2111. Minor product: $\mathrm{mp} 96-98{ }^{\circ} \mathrm{C} ;[\alpha]^{20}{ }_{\mathrm{D}}+216^{\circ}\left(\mathrm{c} 0.33, \mathrm{CHCl}_{3}\right)$; IR (film) 1737, $1700 \mathrm{~cm}^{-1} ;{ }^{1} \mathrm{H}$ NMR (300 MHz, $\left.\mathrm{CDCl}_{3}\right) \delta 8.21(\mathrm{~d}, J=8.2 \mathrm{~Hz}, 1 \mathrm{H}), 7.50(\mathrm{~s}, 1 \mathrm{H})$, 
7.27 (t, $J=7.9 \mathrm{~Hz}, 1 \mathrm{H}), 7.07$ (d, $J=7.2 \mathrm{~Hz}, 1 \mathrm{H}), 4.74$ (br d, $J \sim 8.7 \mathrm{~Hz}, 1 \mathrm{H}), 4.54$ (br q, $J$ $\sim 5.6 \mathrm{~Hz}, 1 \mathrm{H}), 3.82(\mathrm{~s}, 3 \mathrm{H}), 3.32(\mathrm{dd}, J=15.4,4.5 \mathrm{~Hz}, 1 \mathrm{H}), 3.06(\mathrm{dd}, J=14.7,12.5 \mathrm{~Hz}$, 1H), $1.66(\mathrm{~s}, 9 \mathrm{H}), 1.50(\mathrm{~d}, J=7.3 \mathrm{~Hz}, 3 \mathrm{H}), 1.16(\mathrm{~s}, 9 \mathrm{H}) ;{ }^{13} \mathrm{C} \mathrm{NMR}\left(300 \mathrm{MHz}, \mathrm{CDCl}_{3}\right) \delta$ $183.5,170.9,151.5,149.1,137.5,135.4,126.8,125.7,125.6,124.3,115.4,114.3,83.8$, 83.2, 56.8, 52.6, 49.5, 28.2, 27.9, 27.6, 21.4; LRMS(ESI) $\mathrm{m} / z$ (relative intensity) 495.1 $\left(72 \%, \mathrm{M}+\mathrm{Na}^{+}\right)$; $\mathrm{HRMS}(\mathrm{ESI}) \mathrm{m} / z$ calcd for $\left[\mathrm{C}_{25} \mathrm{H}_{32} \mathrm{~N}_{2} \mathrm{O}_{7} \mathrm{Na}\right]^{+}: 495.2107$, found 495.2101 .

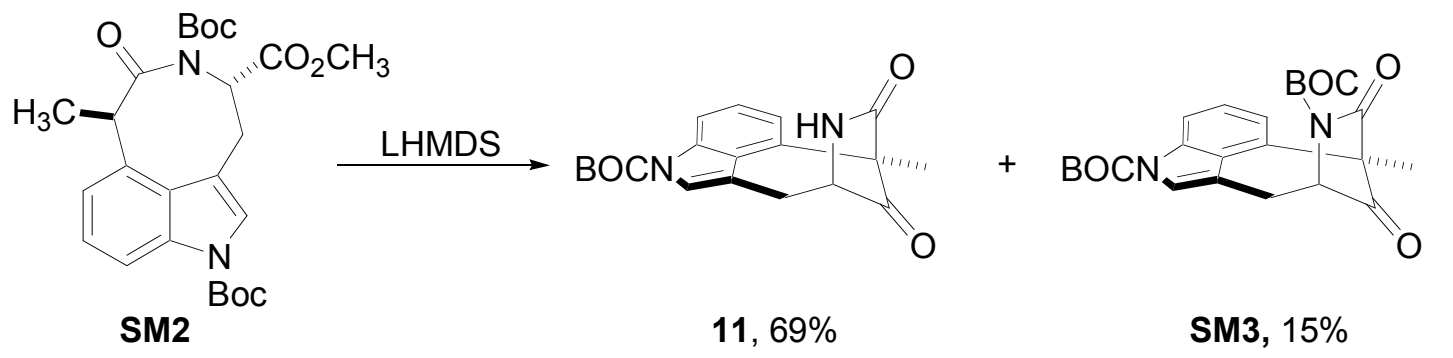

Oxo-amide 11 and oxo-imide SM3. To an ice-cooled solution of trans-SM2 (339 $\mathrm{mg}, 0.72 \mathrm{mmol})$ in THF $(10 \mathrm{~mL})$ was added slowly a $1 \mathrm{M}$ lithium hexamethyldisilazide (LHMDS) solution in THF $(800 \mu \mathrm{L}, 0.80 \mathrm{mmol})$. The reaction mixture was stirred at room temperature for $15 \mathrm{~h}$. The resulting yellow solution was poured into a $1 \mathrm{M}$ aq. $\mathrm{H}_{3} \mathrm{PO}_{4}$ solution $(10 \mathrm{~mL})$ and extracted with ether $(3 \mathrm{x} 20 \mathrm{~mL})$. The combined organic extracts were dried over anhydrous $\mathrm{Na}_{2} \mathrm{SO}_{4}$ and concentrated under reduced pressure. The crude residue was purified by flash chromatography on silica gel (3:2 hexanes/ether and 1:4 hexanes/EtOAc, respectively) to afford the bridging amide $\mathbf{1 1}$ as a white solid (169 mg, 69\%) and its $N$-protected analogue $\mathbf{S M 3}$ as a light yellow solid (46 mg, 15\%) . Oxo-amide 11. $\mathrm{mp} 206-208^{\circ} \mathrm{C} ;[\alpha]^{20}{ }_{\mathrm{D}}+24^{\circ}\left(c \mathrm{0} 0.52, \mathrm{CHCl}_{3}\right)$; IR (film) $3241,1774,1733,1704 \mathrm{~cm}^{-1} ;{ }^{1} \mathrm{H}$ NMR $\left(300 \mathrm{MHz}, \mathrm{CDCl}_{3}\right) \delta 8.17(\mathrm{~d}, J=8.2 \mathrm{~Hz}, 1 \mathrm{H})$, $7.48(\mathrm{~s}, 1 \mathrm{H}), 7.46(\mathrm{br} \mathrm{s}, 1 \mathrm{H}), 7.34(\mathrm{t}, J=8.0 \mathrm{~Hz}, 1 \mathrm{H}), 7.24(\mathrm{dd}, J=7.7,0.9 \mathrm{~Hz}, 1 \mathrm{H}), 4.46$ (ddd, $J=4.1,3.0,1.3 \mathrm{~Hz}, 1 \mathrm{H}), 3.27$ (ddd, $J=17.0,4.0,1.0 \mathrm{~Hz}, 1 \mathrm{H}), 3.13$ (ddd, $J=17.0$, 
2.9, $2.0 \mathrm{~Hz}, 1 \mathrm{H}), 1.73$ (s, 3H), 1.62 (s, 9H); $\left.{ }^{13} \mathrm{C} \mathrm{NMR} \mathrm{(300} \mathrm{MHz,} \mathrm{CDCl}_{3}\right) \delta$ 209.8, 175.1, $149.0,135.8,128.8,126.5,124.9,124.7,118.9,115.3,113.8,84.1,60.9,56.2,29.3,28.1$, 12.8; LRMS(ESI) $m / z$ (relative intensity) $363.1\left(68 \%, \mathrm{M}+\mathrm{Na}^{+}\right)$; HRMS (ESI) $m / z$ calcd for $\left[\mathrm{C}_{19} \mathrm{H}_{20} \mathrm{~N}_{2} \mathrm{O}_{4} \mathrm{Na}\right]^{+}: 363.1321$, found 363.1317. Oxo-imide SM3. mp 122-124 ${ }^{\circ} \mathrm{C}$; $[\alpha]_{\mathrm{D}}^{20}+10^{\circ}\left(c \mathrm{1} 1.00, \mathrm{CHCl}_{3}\right)$; IR (film) 1799, 1764, $1742 \mathrm{~cm}^{-1} ;{ }^{1} \mathrm{H}$ NMR $(300 \mathrm{MHz}$, $\left.\mathrm{CDCl}_{3}\right) \delta 8.20(\mathrm{~d}, J=8.2 \mathrm{~Hz}, 1 \mathrm{H}), 7.55(\mathrm{~s}, 1 \mathrm{H}), 7.35(\mathrm{t}, J=8.0 \mathrm{~Hz}, 1 \mathrm{H}), 7.25(\mathrm{dd}, J=$ 7.6, $0.9 \mathrm{~Hz}, 1 \mathrm{H}), 4.89$ (dd, $J=4.2,2.9 \mathrm{~Hz}, 1 \mathrm{H}), 3.70$ (ddd, $J=17.1,4.3,0.9 \mathrm{~Hz}, 1 \mathrm{H})$, $3.16(\mathrm{ddd}, J=17.1,2.8,2.0 \mathrm{~Hz}, 1 \mathrm{H}), 1.78(\mathrm{~s}, 3 \mathrm{H}), 1.65(\mathrm{~s}, 9 \mathrm{H}), 1.52(\mathrm{~s}, 9 \mathrm{H}) ;{ }^{13} \mathrm{C}$ NMR (300 MHz, $\left.\mathrm{CDCl}_{3}\right) \delta$ 207.6, 170.6, 149.3, 149.0, 135.8, 127.6, 126.3, 124.9, 124.7, 118.8, $115.5,113.2,84.4,84.2,64.8,59.1,28.1,27.9,26.9,13.4$; LRMS(ESI) $m / z$ (relative intensity) $463.2\left(65 \%, \mathrm{M}+\mathrm{Na}^{+}\right)$; HRMS (ESI) $\mathrm{m} / z$ calcd for $\left[\mathrm{C}_{24} \mathrm{H}_{28} \mathrm{~N}_{2} \mathrm{O}_{6} \mathrm{Na}\right]^{+}: 463.1845$, found 463.1841 .

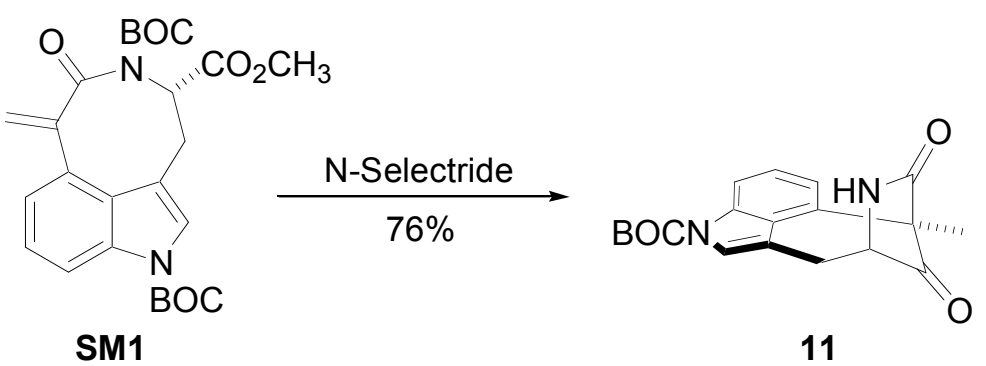

Oxo-amide 11. A solution of indole SM1 (604 mg, $1.28 \mathrm{mmol})$ in THF (15 mL) was cooled to $-78^{\circ} \mathrm{C}$ and a $1 \mathrm{M}$ solution $N$-selectride in THF $(1.42 \mathrm{~mL}, 1.42 \mathrm{mmol})$ was added slowly. The reaction mixture was stirred at $-78{ }^{\circ} \mathrm{C}$ for $1 \mathrm{~h}$ and then at room temperature for $4 \mathrm{~h}$. The resulting red solution was poured into ice water $(15 \mathrm{~mL})$ and extracted with EtOAc $(3 \times 25 \mathrm{~mL})$. The combined organic extracts were dried over anhydrous $\mathrm{Na}_{2} \mathrm{SO}_{4}$ and concentrated under reduced pressure. The crude residue was 
purified by flash chromatography on silica gel (1:3 hexanes/EtOAc and 100\% EtOAc, respectively) to afford the bridging amide $\mathbf{1 1}$ as a light yellow solid (333 mg, 76\%).

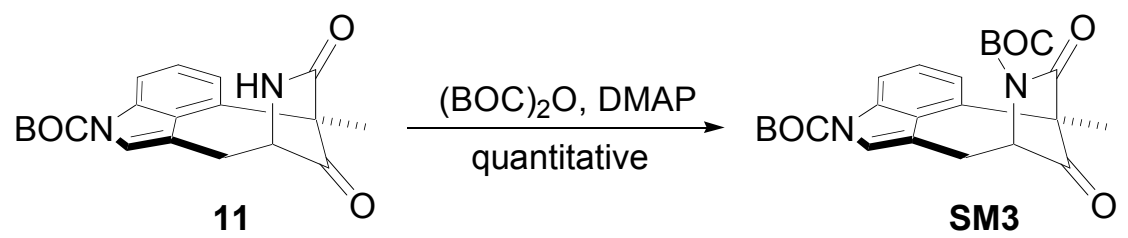

Oxo-imide SM3. A solution of di-tert-butyldicarbonate (1.92 g, $8.80 \mathrm{mmol})$ in $\mathrm{CH}_{3} \mathrm{CN}(20 \mathrm{~mL})$ was cannulated into a suspension of amide $11(1.49 \mathrm{~g}, 4.38 \mathrm{mmol})$ and 4-dimethylaminopyridine (54 mg, $0.44 \mathrm{mmol})$ in $\mathrm{CH}_{3} \mathrm{CN}(20 \mathrm{~mL})$. After the addition was complete, the indole $\mathbf{1 1}$ slowly became soluble and it all went into solution in less than $10 \mathrm{~min}$. The reaction mixture was stirred at room temperature for $1 \mathrm{~h}$. The resulting slightly yellow solution was poured into ice water $(30 \mathrm{~mL})$ and extracted with ether $(3 \mathrm{x}$ $30 \mathrm{~mL})$. The organic extracts were combined, washed with brine solution $(30 \mathrm{~mL})$, dried over anhydrous $\mathrm{Na}_{2} \mathrm{SO}_{4}$, and concentrated under reduced pressure. The crude residue was purified by flash chromatography on silica gel (3:2 hexanes/ether) to afford the desired protected product as a white solid $(2.09 \mathrm{~g}$, quantitative).

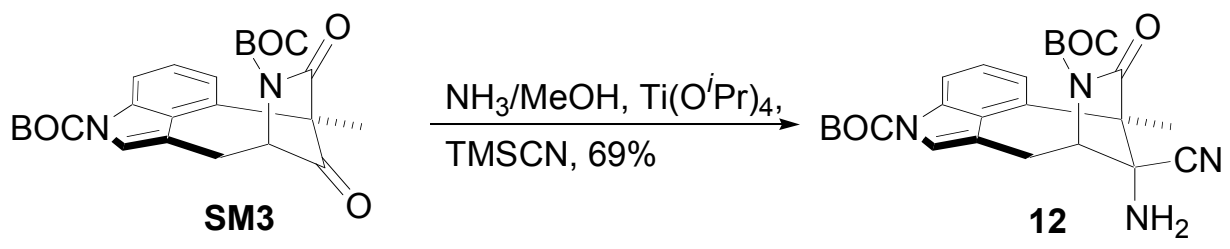

Cyanoamine 12. To a suspension of amide SM3 (1.00 g, $2.28 \mathrm{mmol})$ in $\mathrm{MeOH}$ (23 mL) was added saturated $\mathrm{NH}_{3} / \mathrm{MeOH}(3.4 \mathrm{~mL}$ ) followed by titanium isopropoxide $(810 \mu \mathrm{L}, 2.74 \mathrm{mmol})$. The reaction mixture was stirred at room temperature for $1 \mathrm{~h}$, and then trimethylsilylcyanide $(1.20 \mathrm{~mL}, 9.00 \mathrm{mmol})$ was added. The reaction mixture was stirred for an additional $4 \mathrm{~h}$. The resulting yellow solution was poured into ice water (25 $\mathrm{mL})$ and extracted with $\mathrm{CH}_{2} \mathrm{Cl}_{2}(3 \times 75 \mathrm{~mL})$. The combined organic extracts were dried 
over anhydrous $\mathrm{Na}_{2} \mathrm{SO}_{4}$ and concentrated under reduced pressure. The crude residue was purified by flash chromatography on silica gel (1:2 hexanes/ether) to afford the cyanoamine 12 as a white solid $(736 \mathrm{mg}, 69 \%)$. mp $154-156{ }^{\circ} \mathrm{C} ;[\alpha]^{20}{ }_{\mathrm{D}}+56^{\circ}(c 0.80$, $\mathrm{CHCl}_{3}$ ); IR (film) 3381, 3320, 2254, 1785, $1736 \mathrm{~cm}^{-1} ;{ }^{1} \mathrm{H} \mathrm{NMR}\left(300 \mathrm{MHz}, \mathrm{CDCl}_{3}\right) \delta$ $8.22(\mathrm{dd}, J=6.4,2.6 \mathrm{~Hz}, 1 \mathrm{H}), 7.53(\mathrm{~s}, 1 \mathrm{H}), 7.38-7.31(\mathrm{~m}, 2 \mathrm{H}), 4.96(\mathrm{dd}, J=3.7,3.0 \mathrm{~Hz}$, 1H), 3.69 (ddd, $J=17.8,3.9,0.9 \mathrm{~Hz}, 1 \mathrm{H}), 3.24$ (ddd, $J=17.8,2.6,2.1 \mathrm{~Hz}, 1 \mathrm{H}), 2.05$ (s, 3H), 1.74 (s, 2H), 1.65 (s, 9H), 1.52 (s, 9H); $\left.{ }^{13} \mathrm{C} \mathrm{NMR} \mathrm{(300} \mathrm{MHz,} \mathrm{CDCl}_{3}\right) \delta$ 170.8, 149.4, 149.0, 135.6, 127.6, 127.1, 125.0, 124.8, 122.3, 121.6, 116.2, 113.9, 84.4, 84.2, 62.9, $60.5,56.7,28.2,27.9,25.1,18.9$; LRMS(ESI) $\mathrm{m} / \mathrm{z}$ (relative intensity) 489.1 (88\%, $\mathrm{M}+\mathrm{Na}^{+}$); HRMS (ESI) $m / z$ calcd for $\left[\mathrm{C}_{25} \mathrm{H}_{30} \mathrm{~N}_{4} \mathrm{O}_{5} \mathrm{Na}\right]^{+}: 489.2114$, found 489.2110 .

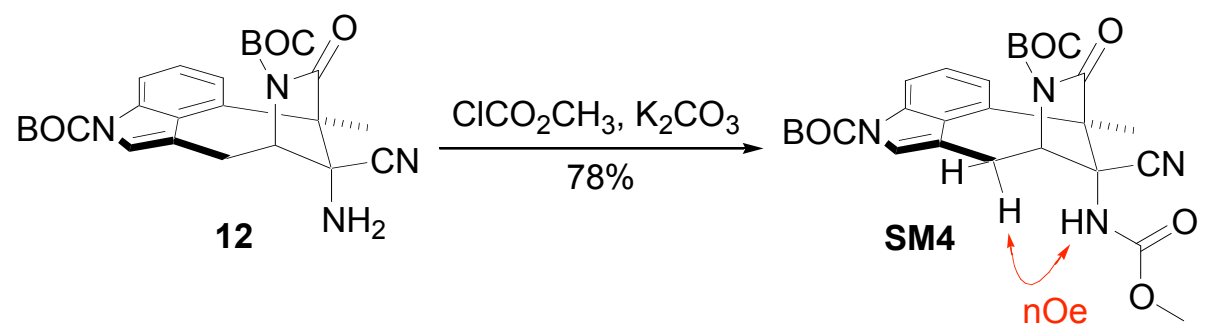

$N$-Moc Cyanoamine SM4. To a mixture of cyanoamine 12 (602 mg, $1.29 \mathrm{mmol})$ and $\mathrm{K}_{2} \mathrm{CO}_{3}$ (357 mg, $\left.2.58 \mathrm{mmol}\right)$ was added THF $(13 \mathrm{~mL})$ followed by methylchloroformate $(220 \mu \mathrm{L}, 2.61 \mathrm{mmol})$. The reaction mixture was heated to reflux for $20 \mathrm{~h}$. The resulting yellow solution was allowed to cool to room temperature, poured into ice water $(15 \mathrm{~mL})$, and extracted with $\mathrm{CH}_{2} \mathrm{Cl}_{2}(3 \times 15 \mathrm{~mL})$. The combined organic layers were dried over anhydrous $\mathrm{Na}_{2} \mathrm{SO}_{4}$ and concentrated under reduced pressure. The crude residue was purified by flash chromatography on silica gel (1:2 hexanes/ether) to afford the desired protected amine as a white solid $(527 \mathrm{mg}, 78 \%)$. mp $164-166{ }^{\circ} \mathrm{C}$; $[\alpha]^{20}{ }_{\mathrm{D}}+43^{\circ}\left(c \mathrm{c} 0.76, \mathrm{CHCl}_{3}\right)$; IR (film) 3301, 2256, 1790, $1732 \mathrm{~cm}^{-1} ;{ }^{1} \mathrm{H}$ NMR (400 MHz, 
$\left.\mathrm{CDCl}_{3}\right) \delta 8.24$ (app.t, $\left.J=4.3 \mathrm{~Hz}, 1 \mathrm{H}\right), 7.51(\mathrm{~s}, 1 \mathrm{H}), 7.38-7.28(\mathrm{~m}, 2 \mathrm{H}), 5.38$ (br s, $\left.1 \mathrm{H}\right)$, 5.19 (br s, 1H), 3.65 (d, $J=18.8 \mathrm{~Hz}, 1 \mathrm{H}), 3.60$ (s, 3H), 2.98 (d, $J=17.9 \mathrm{~Hz}, 1 \mathrm{H}), 2.08$ (s, 3H), 1.65 (s, 9H), 1.49 (s, 9H); ${ }^{13} \mathrm{C}$ NMR $\left(300 \mathrm{MHz}, \mathrm{CDCl}_{3}\right) \delta$ 169.2, 155.1, 148.8, $148.7,135.5,127.4,125.5,124.8,124.6,122.5,118.0,116.6,113.8,84.3,84.1,61.9$, 60.4, 55.4, 52.8, 27.8, 27.6, 24.9, 19.1; LRMS(ESI) $\mathrm{m} / \mathrm{z}$ (relative intensity) $547.2(60 \%$, $\mathrm{M}+\mathrm{Na}^{+}$); HRMS (ESI) $m / z$ calcd for $\left[\mathrm{C}_{27} \mathrm{H}_{32} \mathrm{~N}_{4} \mathrm{O}_{7} \mathrm{Na}\right]^{+}: 547.2169$, found 547.2165.

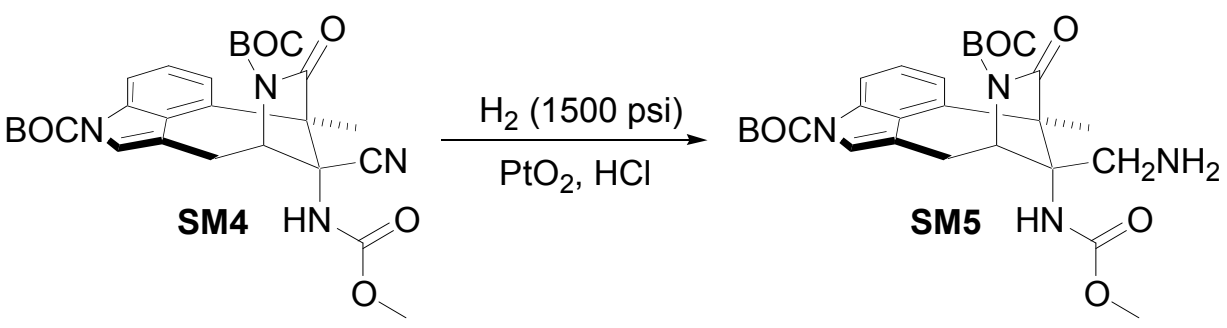

$\mathrm{N}$-Moc Diamine SM5. To a solution of cyanoamine SM4 $(669 \mathrm{mg}, 1.28 \mathrm{mmol})$ in a 1:1 mixture of $\mathrm{MeOH}$ and $\mathrm{EtOH}(40 \mathrm{~mL})$ was added $\mathrm{PtO}_{2}(145 \mathrm{mg}, 0.64 \mathrm{mmol})$ followed by a $10 \%$ solution of $\mathrm{HCl}$ in $\mathrm{MeOH}(1.05 \mathrm{~mL}, 1.27 \mathrm{mmol})$. The reaction mixture was placed in a sealable metal container equipped with a gas inlet and pressure gauge and pressurized with $\mathrm{H}_{2}$ at 1500 psi for $17 \mathrm{~h}$. The resulting clear and colorless solution with settled black particles was filtered through a Celite pad and the filtrate was poured into ice water $(20 \mathrm{~mL})$ and extracted with $\mathrm{CH}_{2} \mathrm{Cl}_{2}(3 \times 30 \mathrm{~mL})$. The combined organic layers were dried over anhydrous $\mathrm{Na}_{2} \mathrm{SO}_{4}$ and concentrated under reduced pressure to give the crude residue as a white solid $(674 \mathrm{mg})$. The crude residue was carried on to the next step without purification. IR (film) 3401, 1782, $1731 \mathrm{~cm}^{-1} ;{ }^{1} \mathrm{H}$ NMR (300 MHz, $\left.\mathrm{CDCl}_{3}\right) \delta 8.20(\mathrm{~d}, J=7.9 \mathrm{~Hz}, 1 \mathrm{H}), 7.47(\mathrm{~s}, 1 \mathrm{H}), 7.34-7.27(\mathrm{~m}, 2 \mathrm{H}), 4.83$ (br s, 1H), $4.77(\mathrm{~s}, 1 \mathrm{H}), 3.76(\mathrm{~d}, J=13.7 \mathrm{~Hz}, 1 \mathrm{H}), 3.61(\mathrm{dd}, J=18.1,3.3 \mathrm{~Hz}, 1 \mathrm{H}), 3.48$ (s, 3H), $3.24(\mathrm{~d}, J=13.7 \mathrm{~Hz}, 1 \mathrm{H}), 3.03(\mathrm{~d}, J=17.9 \mathrm{~Hz}, 1 \mathrm{H}), 1.81(\mathrm{~s}, 3 \mathrm{H}), 1.65(\mathrm{~s}, 9 \mathrm{H}), 1.49$ (s, 9H), 1.32 (br s, $2 \mathrm{H}) ;{ }^{13} \mathrm{C} \mathrm{NMR}\left(300 \mathrm{MHz}, \mathrm{CDCl}_{3}\right) \delta$ 172.6, 155.9, 149.7, 149.0, 135.6, 
$129.4,128.2,124.3,124.1,121.9,115.7,115.6,83.7,83.2,62.7,61.2,56.9,51.9,46.2$, 27.9, 27.8, 25.8, 16.2; LRMS(ESI) $\mathrm{m} / z$ (relative intensity) $529.2\left(72 \%, \mathrm{M}+\mathrm{H}^{+}\right)$; HRMS (ESI) $m / z$ calcd for $\left[\mathrm{C}_{27} \mathrm{H}_{37} \mathrm{~N}_{4} \mathrm{O}_{7}\right]^{+}: 529.2662$, found 529.2656 .

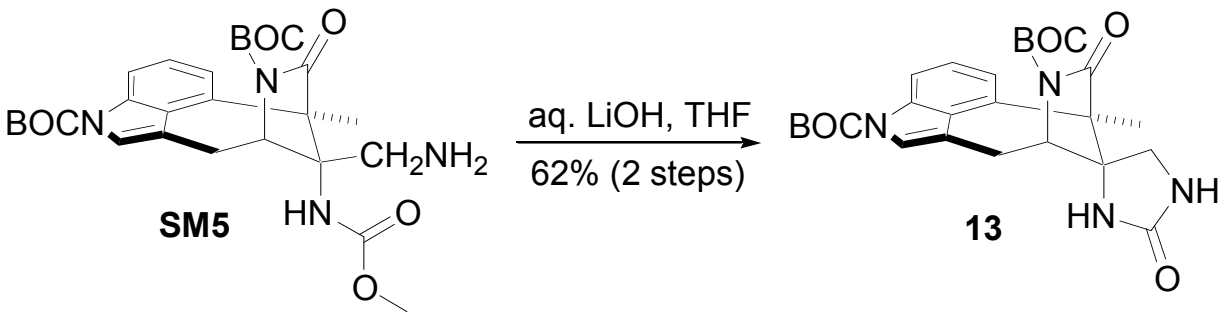

Cyclic Urea 13. To a solution of amine SM5 (674 mg, $1.28 \mathrm{mmol})$ in THF (25 $\mathrm{mL}$ ) was added a deoxygenated $1 \mathrm{M}$ aq. $\mathrm{LiOH}$ solution $(5.1 \mathrm{~mL}, 5.1 \mathrm{mmol})$. The reaction mixture was heated to reflux for $17 \mathrm{~h}$. The resulting slightly yellow solution was allowed to cool to room temperature and then poured into ice water and extracted with $\mathrm{CH}_{2} \mathrm{Cl}_{2}$ (3 x $30 \mathrm{~mL}$ ). The combined organic layers were dried over anhydrous $\mathrm{Na}_{2} \mathrm{SO}_{4}$ and concentrated under reduced pressure to give a yellow solid $(600 \mathrm{mg})$. Cold ether $(10 \mathrm{~mL})$ was added to the resulting yellow solid to dissolve impurities and the desired cyclic urea was collected by filtration $(394 \mathrm{mg}, 62 \%$ over 2 steps $) \cdot \mathrm{mp} 248^{\circ} \mathrm{C}(\mathrm{dec}) ;[\alpha]^{20}{ }_{\mathrm{D}}+88^{\circ}(\mathrm{c}$ 1.00, DMSO); IR (film) 3344, 1782, 1730, $1687 \mathrm{~cm}^{-1} ;{ }^{1} \mathrm{H}$ NMR (300 MHz, DMSO-d6) $\delta$ $8.45(\mathrm{~s}, 1 \mathrm{H}), 8.08(\mathrm{~d}, J=8.2 \mathrm{~Hz}, 1 \mathrm{H}), 7.82(\mathrm{~s}, 1 \mathrm{H}), 7.73(\mathrm{~s}, 1 \mathrm{H}), 7.58(\mathrm{~d}, J=7.9,1 \mathrm{H})$, $7.30(\mathrm{t}, J=8.1 \mathrm{~Hz}, 1 \mathrm{H}), 4.27(\mathrm{dd}, J=12.4,3.1 \mathrm{~Hz}, 1 \mathrm{H}), 3.83(\mathrm{dd}, J=16.1,2.9 \mathrm{~Hz}, 1 \mathrm{H})$, $3.60(\mathrm{~d}, J=10.4 \mathrm{~Hz}, 1 \mathrm{H}), 3.34$ (s, 3H), $3.21(\mathrm{~d}, J=8.9 \mathrm{~Hz}, 1 \mathrm{H}), 2.86(\mathrm{t}, J=13.5 \mathrm{~Hz}, 1 \mathrm{H})$,

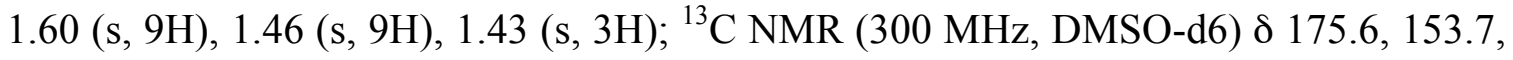
$150.9,148.7,135.4,132.5,127.4,124.4,124.2,123.8,114.3,113.6,83.8$ 81.7, 65.9, 55.1, 53.7, 48.6, 27.8, 27.7, 26.4, 19.4; LRMS(ESI) $\mathrm{m} / \mathrm{z}$ (relative intensity) 519.2 (52\%, $\mathrm{M}+\mathrm{Na}^{+}$); HRMS (ESI) $m / z$ calcd for $\left[\mathrm{C}_{26} \mathrm{H}_{32} \mathrm{~N}_{4} \mathrm{O}_{6} \mathrm{Na}\right]^{+}: 519.2220$, found 519.2227. 


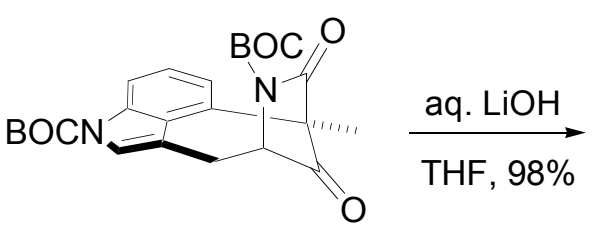

SM3

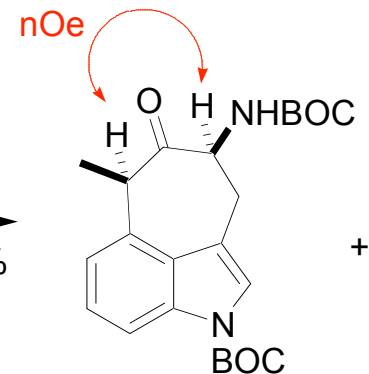

cis-15

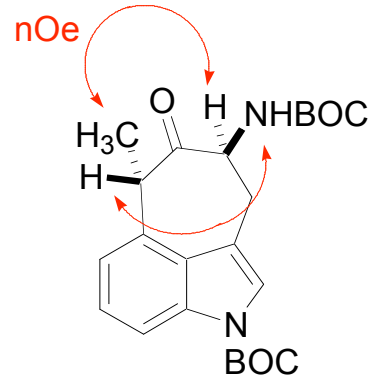

trans-15

tert-Butyl 8-tert-Butoxycarbonylamino-6-methyl-7-oxo-6,7,8,9-tetrahydro-2-

aza benzo $[c d]$ azulene-2 Carboxylate (15). To an ice-cooled solution of amide SM3 (1.029 g, $2.34 \mathrm{mmol})$ in THF (47 mL) was added a deoxygenated $1 \mathrm{M}$ aq. LiOH solution (4.7 $\mathrm{mL}, 4.7 \mathrm{mmol})$. The reaction mixture was stirred in an ice bath for $1 \mathrm{~h}$ and then at room temperature for $14 \mathrm{~h}$. The resulting slightly yellow solution was poured into ice water $(30 \mathrm{~mL})$ and extracted with ether $(3 \times 40 \mathrm{~mL})$. The combined organic extracts were dried over anhydrous $\mathrm{Na}_{2} \mathrm{SO}_{4}$ and concentrated under reduced pressure. The crude residue was purified by flash chromatography on silica gel ( $3: 2$ hexanes/ether) to afford a $\sim$ 2:1 diastereomeric mixture of ketones 15 as a white solid (951 mg, 98\%). Major product (cis-15): $\mathrm{mp} 163-164{ }^{\circ} \mathrm{C} ;[\alpha]^{20}{ }_{\mathrm{D}}+29^{\circ}\left(c 1.00, \mathrm{CHCl}_{3}\right)$; IR (film) $3416,1729 \mathrm{~cm}^{-1}$; ${ }^{1} \mathrm{H}$ NMR $\left(300 \mathrm{MHz}, \mathrm{CDCl}_{3}\right) \delta 7.99(\mathrm{~d}, J=8.1 \mathrm{~Hz}, 1 \mathrm{H}), 7.43(\mathrm{~s}, 1 \mathrm{H}), 7.26(\mathrm{t}, J=7.9 \mathrm{~Hz}$, 1H), $7.01(\mathrm{~d}, J=7.5 \mathrm{~Hz}, 1 \mathrm{H}), 5.42($ br s, $1 \mathrm{H}), 4.74$ (br dd, $J \sim 9.2,4.6 \mathrm{~Hz}, 1 \mathrm{H}), 4.62(\mathrm{q}, J$ $=6.8 \mathrm{~Hz}, 1 \mathrm{H}), 3.60(\mathrm{dd}, J=15.7,4.6 \mathrm{~Hz}, 1 \mathrm{H}), 3.17(\mathrm{dd}, J=15.8,4.2 \mathrm{~Hz}, 1 \mathrm{H}), 1.65(\mathrm{~s}$, 9H), $1.65(\mathrm{~d}, J=6.9 \mathrm{~Hz}, 3 \mathrm{H}), 1.45(\mathrm{~s}, 9 \mathrm{H}) ;{ }^{13} \mathrm{C} \mathrm{NMR}\left(300 \mathrm{MHz}, \mathrm{CDCl}_{3}\right) \delta 206.1,155.5$, $149.2,135.1,130.7,128.3,125.0,123.0,118.7,116.1,114.1,83.5,79.5,61.4,45.7,28.2$ 28.0, 27.6, 14.4; LRMS(ESI) $m / z$ (relative intensity) $437.2\left(100 \%, \mathrm{M}+\mathrm{Na}^{+}\right)$; HRMS (ESI) $m / z$ calcd for $\left[\mathrm{C}_{23} \mathrm{H}_{30} \mathrm{~N}_{2} \mathrm{O}_{5} \mathrm{Na}\right]^{+}: 437.2052$, found 457.2042. Anal. Calcd for $\mathrm{C}_{23} \mathrm{H}_{30} \mathrm{~N}_{2} \mathrm{O}_{5}$ : C, 66.65; H, 7.30; N, 6.76. Found: C, 66.59; H, 7.32; N, 6.76. Minor product (trans-15): 
mp 170-171 ${ }^{\circ} \mathrm{C} ;[\alpha]^{20}{ }_{\mathrm{D}}+90^{\circ}\left(c 1.00, \mathrm{CHCl}_{3}\right)$; IR (film) $3385,1720 \mathrm{~cm}^{-1} ;{ }^{1} \mathrm{H}$ NMR (400 $\left.\mathrm{MHz}, \mathrm{CDCl}_{3}\right) \delta 8.06(\mathrm{~d}, J=8.1 \mathrm{~Hz}, 1 \mathrm{H}), 7.48(\mathrm{~s}, 1 \mathrm{H}), 7.30(\mathrm{t}, J=7.9 \mathrm{~Hz}, 1 \mathrm{H}), 7.08(\mathrm{~d}, J$ $=7.6 \mathrm{~Hz}, 1 \mathrm{H}), 5.52(\mathrm{br} \mathrm{s}, 1 \mathrm{H}), 4.50(\mathrm{q}, J=7.0 \mathrm{~Hz}, 1 \mathrm{H}), 4.43(\mathrm{br} \mathrm{dd}, J \sim 11.9,5.7 \mathrm{~Hz}$, 1H), $3.29(\mathrm{dd}, J=14.7,2.7 \mathrm{~Hz}, 1 \mathrm{H}), 3.19(\mathrm{ddd}, J=15.2,10.2,1.4 \mathrm{~Hz}, 1 \mathrm{H}), 1.66(\mathrm{~s}, 9 \mathrm{H})$, $1.64(\mathrm{~d}, J=7.0 \mathrm{~Hz}, 3 \mathrm{H}), 1.45(\mathrm{~s}, 9 \mathrm{H}) ;{ }^{13} \mathrm{C}$ NMR $\left(300 \mathrm{MHz}, \mathrm{CDCl}_{3}\right) \delta 208.8,155.4$, $149.4,135.6,130.7,128.0,124.9,123.1,120.4,116.1,113.9,83.7,80.0,58.9,49.0,29.3$, 28.3, 28.1, 16.4; LRMS(ESI) $m / z$ (relative intensity) $437.2\left(100 \%, \mathrm{M}+\mathrm{Na}^{+}\right)$; HRMS (ESI) $m / z$ calcd for $\left[\mathrm{C}_{23} \mathrm{H}_{30} \mathrm{~N}_{2} \mathrm{O}_{5} \mathrm{Na}\right]^{+}: 437.2052$, found 437.2060 .
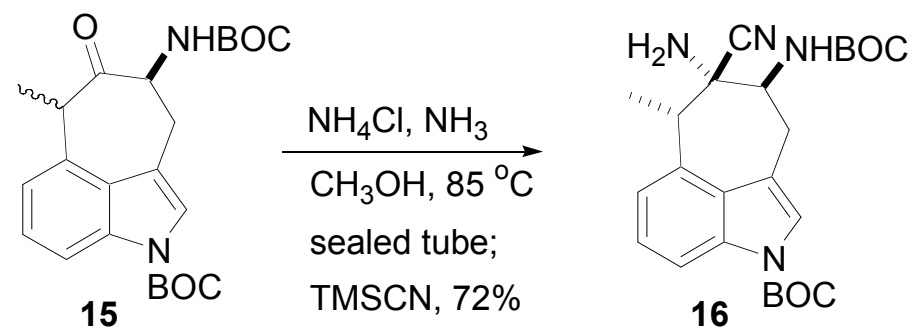

( \pm -tert-Butyl 7-Amino-8-tert-butoxycarbonylamino-7-cyano-6-methyl6,7,8,9- tetrahydro-2-aza-benzo $[c d]$ azulene-2 Carboxylate (16). A solution of the diastereomeric mixture of ketones 15 (645 mg, $1.56 \mathrm{mmol})$ and $\mathrm{NH}_{4} \mathrm{Cl}(166 \mathrm{mg}, 3.10$ mmol) in saturated $\mathrm{NH}_{3} / \mathrm{MeOH}(15 \mathrm{~mL})$ in a sealed tube was heated to $85{ }^{\circ} \mathrm{C}$ for $4 \mathrm{~h}$. The reaction mixture was allowed to cool to room temperature and trimethylsilylcyanide (830 $\mu \mathrm{L}, 6.22 \mathrm{mmol}$ ) was added. The reaction mixture was stirred in a sealed tube at room temperature for $14 \mathrm{~h}$. The resulting yellow suspension was diluted with $\mathrm{CH}_{2} \mathrm{Cl}_{2}(25$ $\mathrm{mL})$ and then poured into ice water $(20 \mathrm{~mL})$. The organic layer was separated and the aqueous layer was extracted with $\mathrm{CH}_{2} \mathrm{Cl}_{2}(2 \times 25 \mathrm{~mL})$. The combined organic layers were dried over anhydrous $\mathrm{Na}_{2} \mathrm{SO}_{4}$ and concentrated under reduced pressure. The crude residue was purified by flash chromatography on silica gel (2:1 hexanes/EtOAc and 
$100 \%$ EtOAc, respectively) to afford the cyanoamine $\mathbf{1 6}$ as a light yellow solid (494 mg, 72\%). mp 152-154 ${ }^{\circ} \mathrm{C} ;[\alpha]^{20}{ }_{\mathrm{D}}-0.8^{\circ}\left(c 1.00, \mathrm{CHCl}_{3}\right)$; IR (film) $3326,2252,1727 \mathrm{~cm}^{-1} ;{ }^{1} \mathrm{H}$ NMR $\left(300 \mathrm{MHz}, \mathrm{CDCl}_{3}\right) \delta 8.00(\mathrm{~d}, J=8.0 \mathrm{~Hz}, 1 \mathrm{H}), 7.40(\mathrm{~s}, 1 \mathrm{H}), 7.27(\mathrm{t}, J=7.9 \mathrm{~Hz}$, $1 \mathrm{H}), 7.06(\mathrm{~d}, J=7.4 \mathrm{~Hz}, 1 \mathrm{H}), 5.16(\mathrm{br} \mathrm{d}, J=9.9,1 \mathrm{H}), 4.0(\operatorname{td}, J=9.8,2.8 \mathrm{~Hz}, 1 \mathrm{H}), 3.70$ (q, $J=7.1 \mathrm{~Hz}, 1 \mathrm{H}), 3.24(\mathrm{dd}, J=15.5,9.5 \mathrm{~Hz}, 1 \mathrm{H}), 3.05(\mathrm{dd}, J=15.7,2.4 \mathrm{~Hz}, 1 \mathrm{H}), 2.05$ (br s, 2H), 1.66 (s, 9H), 1.62 (d, $J=7.2 \mathrm{~Hz}, 3 \mathrm{H}), 1.45$ (s, 9H); ${ }^{13} \mathrm{C}$ NMR (300 MHz, $\left.\mathrm{CDCl}_{3}\right) \delta 155.7,149.5,135.4,132.0,129.1,124.9,122.7,122.3,121.8,116.7,114.5$, 83.7, 80.7, 65.2, 57.6, 43.6, 30.4, 28.3, 28.2, 15.6; LRMS(ESI) $\mathrm{m} / z$ (relative intensity) $441.2\left(35 \%, \mathrm{M}+\mathrm{H}^{+}\right)$; HRMS (ESI) $\mathrm{m} / z$ calcd for $\left[\mathrm{C}_{24} \mathrm{H}_{33} \mathrm{~N}_{4} \mathrm{O}_{4}\right]^{+}: 441.2502$, found 441.2497.
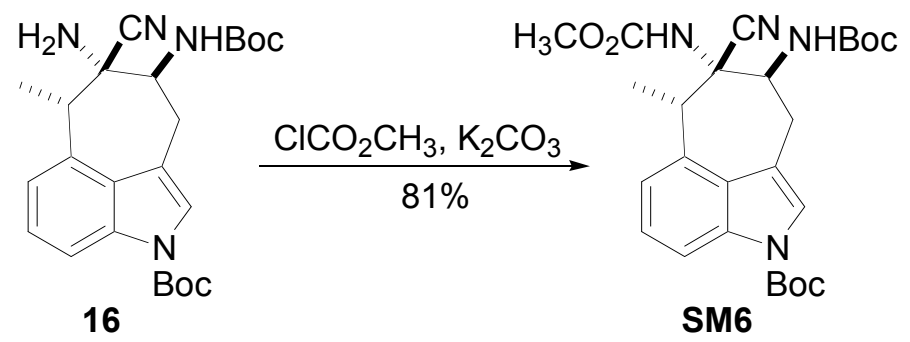

( \pm -tert-Butyl 8-tert-Butoxycarbonylamino-7-cyano-7-methoxycarbonylamino -6-methyl-6,7,8,9-tetrahydro-2-aza-benzo $[c d]$ azulene-2 Carboxylate (SM6). To a mixture of cyanoamine $16(84 \mathrm{mg}, 0.19 \mathrm{mmol})$ and $\mathrm{K}_{2} \mathrm{CO}_{3}(53 \mathrm{mg}, 0.38 \mathrm{mmol})$ was added THF $(4 \mathrm{~mL})$ followed by methylchloroformate $(100 \mu \mathrm{L}, 1.19 \mathrm{mmol})$. The reaction mixture was heated to reflux and held there for $20 \mathrm{~h}$. The resulting yellow solution was allowed to cool to room temperature, poured into ice water $(10 \mathrm{~mL})$, and extracted with $\mathrm{CH}_{2} \mathrm{Cl}_{2}(3 \times 10 \mathrm{~mL})$. The combined organic layers were dried over anhydrous $\mathrm{Na}_{2} \mathrm{SO}_{4}$ and concentrated under reduced pressure. The crude residue was purified by flash chromatography on silica gel (1:2 ether/hexanes) to afford the desired product SM6 as a 
white solid (77 mg, 81\%). mp 138-140 ${ }^{\circ} \mathrm{C} ;[\alpha]^{20}{ }_{\mathrm{D}}+0.2^{\circ}\left(c 1.00, \mathrm{CHCl}_{3}\right)$; IR (film) 3328 , 2254, 1731, $1689 \mathrm{~cm}^{-1} ;{ }^{1} \mathrm{H}$ NMR (300 MHz, $\left.\mathrm{CDCl}_{3}\right) \delta 8.02(\mathrm{~d}, J=8.0 \mathrm{~Hz}, 1 \mathrm{H}), 7.42$ (s, 1H), 7.27 (t, $J=7.9 \mathrm{~Hz}, 1 \mathrm{H}$ ), 7.10 (d, $J=7.3 \mathrm{~Hz}, 1 \mathrm{H}), 6.50$ (br s, $1 \mathrm{H}$ ), 5.40 (br d, $J=9.1$, $1 \mathrm{H}), 4.43(\mathrm{td}, J=8.5,5.5 \mathrm{~Hz}, 1 \mathrm{H}), 4.42(\mathrm{q}, J=7.3 \mathrm{~Hz}, 1 \mathrm{H}), 3.73(\mathrm{~s}, 3 \mathrm{H}), 3.42$ (dd, $J=$ 16.9, $5.1 \mathrm{~Hz}, 1 \mathrm{H}), 3.16$ (dd, $J=17.1,7.8 \mathrm{~Hz}, 1 \mathrm{H}), 1.65$ (s, 9H), 1.47 (s, 9H), 1.44 (d, $J=$ 7.4, 3H); ${ }^{13} \mathrm{C}$ NMR $\left(300 \mathrm{MHz}, \mathrm{CDCl}_{3}\right) \delta 156.8,155.1,149.3,135.6,133.1,127.9,124.9$, $123.2,123.1,117 . .4,114.4,114.1,83.7,81.4,65.0,53.0,52.4,43.7,30.4,28.08,28.06$, 17.5; LRMS(ESI) $m / z$ (relative intensity) $521.2\left(100 \%, \mathrm{M}^{+\mathrm{Na}^{+}}\right)$; HRMS (ESI) $m / z$ calcd for $\left[\mathrm{C}_{26} \mathrm{H}_{34} \mathrm{~N}_{4} \mathrm{O}_{6} \mathrm{Na}\right]^{+}:$521.2376, found 521.2361. Anal. Calcd for $\mathrm{C}_{26} \mathrm{H}_{34} \mathrm{~N}_{4} \mathrm{O}_{6}$ : C, 62.63; H, 6.87; N, 11.24. Found: C, 62.37; H, 6.97; N, 10.99 .
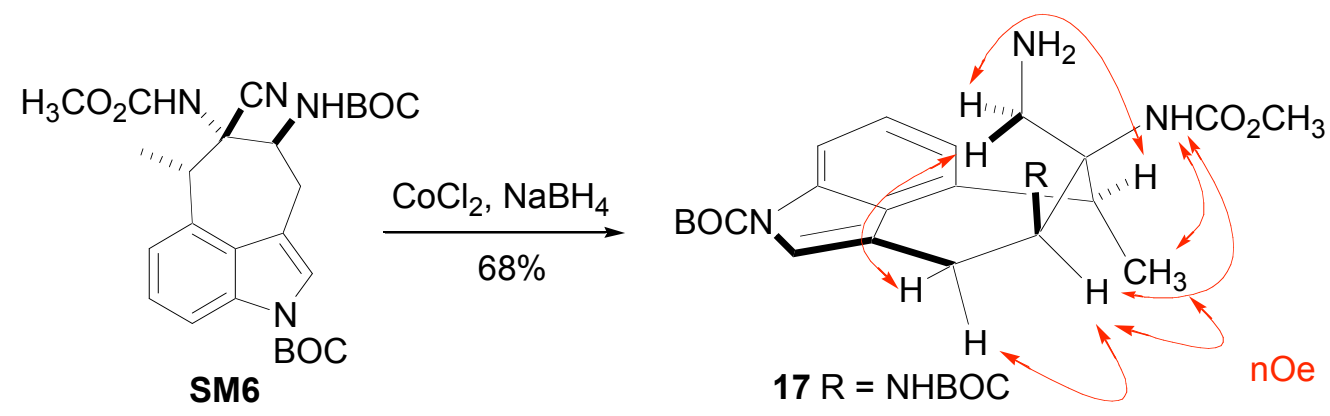

( \pm )-tert-Butyl 7-Aminomethyl-8-tert-butoxycarbonylamino-7-methoxycarbonylamino-6-methyl-6,7,8,9-tetrahydro-2-aza-benzo[cd] azulene-2 Carboxylate (17). To a solution of cyanoamine SM6 (358 $\mathrm{mg}, 0.72 \mathrm{mmol})$ in $\mathrm{MeOH}(30 \mathrm{~mL})$ was added cobalt (II) chloride (2.33 g, $17.9 \mathrm{mmol})$. The resulting dark blue solution was cooled in an ice bath and sodium borohydride $(679 \mathrm{mg}, 17.9 \mathrm{mmol})$ was added in portions. The resulting black suspension was stirred at room temperature for $3 \mathrm{~h}$. It was then recooled in an ice bath and additional sodium borohydride $(679 \mathrm{mg}, 17.9 \mathrm{mmol})$ was added in portions. The reaction mixture was stirred at room temperature for $38 \mathrm{~h}$. The resulting black suspension was diluted with $\mathrm{MeOH}(30 \mathrm{~mL})$ and acidified with $1 \mathrm{M} \mathrm{H}_{3} \mathrm{PO}_{4}$ solution 
$(30 \mathrm{~mL})$. The acidic solution was stirred vigorously for $1 \mathrm{~h}$ until it turned pink and clear. The pink solution was diluted with $\mathrm{CH}_{2} \mathrm{Cl}_{2}(30 \mathrm{~mL})$ and made alkaline by the addition of saturated $\mathrm{NaHCO}_{3}(30 \mathrm{~mL})$. The resulting purple precipitate was filtered off and washed with $\mathrm{CH}_{2} \mathrm{Cl}_{2}(30 \mathrm{~mL})$. The organic layer of the filtrate was separated and the aqueous layer was extracted with $\mathrm{CH}_{2} \mathrm{Cl}_{2}(2 \times 30 \mathrm{~mL})$. The combined organic layers were dried over anhydrous $\mathrm{Na}_{2} \mathrm{SO}_{4}$ and concentrated under reduced pressure. The crude residue was purified by flash chromatography on silica gel (3:2 hexanes/ether and 100\% EtOAC, respectively) to afford the desired amine 17 as a white solid (246 mg, 68\%). mp 124-126 ${ }^{\circ} \mathrm{C} ;[\alpha]^{20}{ }_{\mathrm{D}}-0.2^{\circ}\left(\mathrm{c} 1.00, \mathrm{CHCl}_{3}\right)$; IR (film) $3325,1729 \mathrm{~cm}^{-1} ;{ }^{1} \mathrm{H} \mathrm{NMR}\left(300 \mathrm{MHz}, \mathrm{C}_{6} \mathrm{D}_{6}\right) \delta$ $8.33(\mathrm{~d}, J=7.2 \mathrm{~Hz}, 1 \mathrm{H}), 7.23(\mathrm{~s}, 1 \mathrm{H}), 7.11(\mathrm{t}, J=7.8 \mathrm{~Hz}, 1 \mathrm{H}), 6.96(\mathrm{~d}, J=9.5 \mathrm{~Hz}, 1 \mathrm{H})$, $6.90(\mathrm{~d}, J=6.8 \mathrm{~Hz}, 1 \mathrm{H}), 5.78(\mathrm{~s}, 1 \mathrm{H}), 4.73(\mathrm{td}, J=10.7,4.6 \mathrm{~Hz}, 1 \mathrm{H}), 4.30(\mathrm{q}, J=7.1 \mathrm{~Hz}$, $1 \mathrm{H}), 3.76(\mathrm{~d}, J=13.3 \mathrm{~Hz}, 1 \mathrm{H}), 3.47$ (s, 3H), 3.29 (dd, $J=16.9,4.6 \mathrm{~Hz}, 1 \mathrm{H}), 2.78$ (ddd, $J$ $=16.6,12.1,1.7 \mathrm{~Hz}, 1 \mathrm{H}), 2.64(\mathrm{dd}, J=13.2 \mathrm{~Hz}, 1 \mathrm{H}), 1.52(\mathrm{~s}, 9 \mathrm{H}), 1.39(\mathrm{~s}, 9 \mathrm{H}), 1.24(\mathrm{~d}, J$ $=7.2 \mathrm{~Hz}, 3 \mathrm{H}), 0.59$ (br s, $2 \mathrm{H}) ;{ }^{13} \mathrm{C} \mathrm{NMR}\left(300 \mathrm{MHz}, \mathrm{CDCl}_{3}\right) \delta$ 156.4, 155.1, 149.5, 136.4, $135.8,127.1,124.5,123.7,123.0,115.6,113.1,83.5,79.8,62.8,51.7,51.3,43.8,43.2$, 30.2, 28.5, 28.2, 20.5; LRMS(ESI) $\mathrm{m} / z$ (relative intensity) $503.2\left(100 \%, \mathrm{M}+\mathrm{H}^{+}\right)$; HRMS (ESI) $m / z$ calcd for $\left[\mathrm{C}_{26} \mathrm{H}_{39} \mathrm{~N}_{4} \mathrm{O}_{6}\right]^{+}: 503.2870$, found 503.2874.

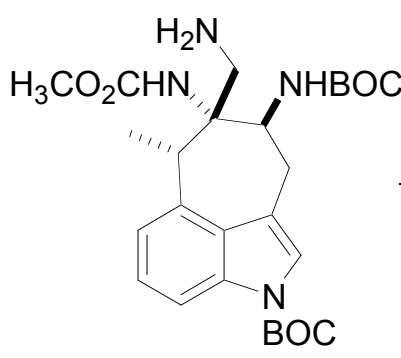

17

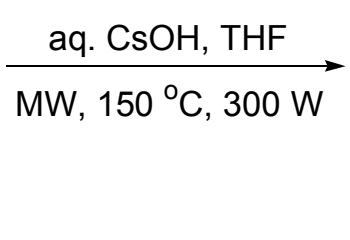

( \pm )-Cyclic Urea SM7. To a mixture of amine $17(30 \mathrm{mg}, 0.060 \mathrm{mmol})$ and cesium hydroxide monohydrate $(301 \mathrm{mg}, 1.79 \mathrm{mmol})$ was added $\mathrm{H}_{2} \mathrm{O}(0.5 \mathrm{~mL})$ followed 
by THF $(2 \mathrm{~mL})$. The reaction mixture was heated to $150{ }^{\circ} \mathrm{C}$ at $300 \mathrm{~W}$ in a microwave reactor for $1 \mathrm{~h}$. The resulting slightly yellow suspension was poured into ice water (10 $\mathrm{mL})$ and extracted with EtOAc $(3 \times 30 \mathrm{~mL})$. The combined organic layers were dried over anhydrous $\mathrm{Na}_{2} \mathrm{SO}_{4}$ and concentrated under reduced pressure to afford a light yellow solid (20 mg, 91\% crude). The crude residue was carried on to the next step without purification. IR (film) $3416,1687 \mathrm{~cm}^{-1} ;{ }^{1} \mathrm{H}$ NMR $\left(300 \mathrm{MHz}, \mathrm{CDCl}_{3}\right) \delta 8.19(\mathrm{br} \mathrm{s}, 1 \mathrm{H})$, $7.23(\mathrm{dd}, J=8.2,0.9 \mathrm{~Hz}, 1 \mathrm{H}), 7.13(\mathrm{t}, J=7.6 \mathrm{~Hz}, 1 \mathrm{H}), 7.02(\mathrm{~s}, 1 \mathrm{H}), 6.93(\mathrm{~d}, J=7.1 \mathrm{~Hz}$, 1H), 5.42 (br s, $1 \mathrm{H}), 4.98$ (br d, $J=8.6 \mathrm{~Hz}, 1 \mathrm{H}), 4.58(\mathrm{~m}, 1 \mathrm{H}), 4.43$ (br s, $1 \mathrm{H}), 3.69$ (q, $J$ $=7.1 \mathrm{~Hz}, 1 \mathrm{H}), 3.49-3.21(\mathrm{~m}, 2 \mathrm{H}), 3.19(\mathrm{~d}, J=9.7,1 \mathrm{H}), 2.84(\mathrm{dd}, J=15.4,11.7 \mathrm{~Hz}, 1 \mathrm{H})$, $1.48(\mathrm{~s}, 9 \mathrm{H}), 1.40$ (d, $J=7.2 \mathrm{~Hz}, 3 \mathrm{H}) ;{ }^{13} \mathrm{C}$ NMR (300 MHz, DMSO-d6) $\delta$ 161.6, 155.9, $136.4,135.2,123.3,122.5,121.1,119.1,109.3,109.2,77.9,65.6,49.3,49.1,44.6,28.4$, 28.2, 19.4; LRMS(ESI) $\mathrm{m} / \mathrm{z}$ (relative intensity) $371.2\left(100 \%, \mathrm{M}+\mathrm{H}^{+}\right)$; HRMS (ESI) $\mathrm{m} / \mathrm{z}$ calcd for $\left[\mathrm{C}_{20} \mathrm{H}_{26} \mathrm{~N}_{4} \mathrm{O}_{3} \mathrm{Na}\right]^{+}:$393.1903, found 393.1898.
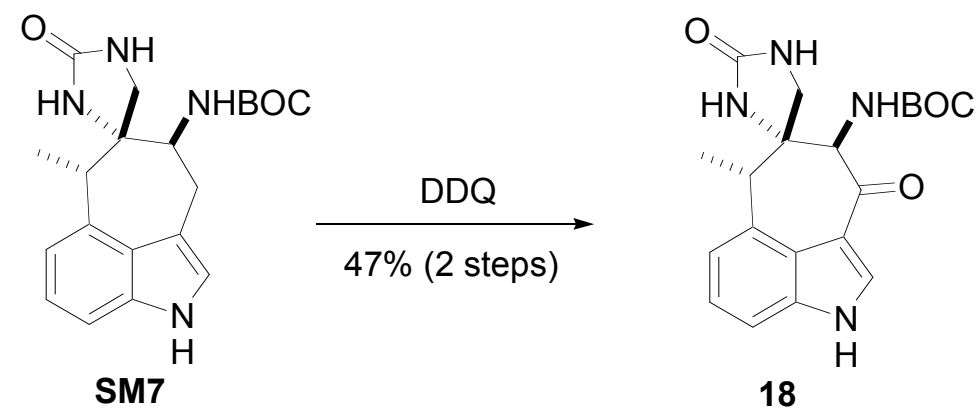

( \pm )-Cyclic Urea 18. To an ice-cooled solution of cyclic urea SM7 (103 mg, 0.28 mmol) in a 10:1 mixture of THF and $\mathrm{H}_{2} \mathrm{O}(5.5 \mathrm{~mL})$ was added 2,3-dichloro-5,6-dicyano1,4-benzoquinone (DDQ) (127 mg, $0.56 \mathrm{mmol})$. The reaction mixture was stirred in an ice bath for $1 \mathrm{~h}$ and then at room temperature for $14 \mathrm{~h}$. The resulting dark yellow solution was concentrated to dryness. $\mathrm{CH}_{2} \mathrm{Cl}_{2}(10 \mathrm{~mL})$ was added to the dark red solid residue followed by a $0.1 \mathrm{M}$ aq. $\mathrm{NaOH}$ solution $(10 \mathrm{~mL})$. The precipitate was filtered off 
and washed with $\mathrm{CH}_{2} \mathrm{Cl}_{2}(10 \mathrm{~mL})$. The organic layer of the filtrate was separated and the aqueous layer was extracted with $\mathrm{CH}_{2} \mathrm{Cl}_{2}(2 \times 25 \mathrm{~mL})$. The combined organic layers were dried over anhydrous $\mathrm{Na}_{2} \mathrm{SO}_{4}$ and concentrated under reduced pressure. The crude residue was purified by flash chromatography on silica gel (100\% EtOAc) to afford the desired product 18 as a yellow solid (55 mg, 47\% over steps). mp $276{ }^{\circ} \mathrm{C}$ (dec.); IR (film) 3408, 1691, $1634 \mathrm{~cm}^{-1}$; ${ }^{1} \mathrm{H}$ NMR (300 MHz, DMSO-d6) $\delta 12.32$ (s, 1H), 8.19 (s, 1H), $7.37(\mathrm{dd}, J=7.2,0.9 \mathrm{~Hz}, 1 \mathrm{H}), 7.20(\mathrm{t}, J=7.6 \mathrm{~Hz}, 1 \mathrm{H}), 7.11(\mathrm{~d}, J=6.9 \mathrm{~Hz}, 1 \mathrm{H})$, $6.95(\mathrm{~d}, J=8.6 \mathrm{~Hz}, 1 \mathrm{H}), 6.44(\mathrm{~s}, 1 \mathrm{H}), 6.02(\mathrm{~s}, 1 \mathrm{H}), 4.89(\mathrm{~d}, J=8.6 \mathrm{~Hz}, 1 \mathrm{H}), 3.53(\mathrm{q}, J=$ $7.2 \mathrm{~Hz}, 1 \mathrm{H}), 3.11(\mathrm{~d}, J=8.6 \mathrm{~Hz}, 1 \mathrm{H}), 2.76(\mathrm{~d}, J=9.1 \mathrm{~Hz}, 1 \mathrm{H}), 1.45(\mathrm{~s}, 9 \mathrm{H}), 1.40(\mathrm{~d}, J=$

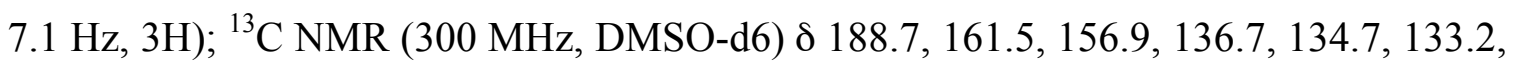
123.1, 122.6, 121.2, 114.2, 110.7, 78.4, 62.4, 61.4, 49.3, 46.8, 28.4, 19.9; LRMS(ESI) $m / z$ (relative intensity) $407.2\left(100 \%, \mathrm{M}+\mathrm{Na}^{+}\right)$; HRMS (ESI) $m / z$ calcd for $\left[\mathrm{C}_{20} \mathrm{H}_{24} \mathrm{~N}_{4} \mathrm{O}_{4} \mathrm{Na}\right]^{+}:$407.1696, found 407.1699. 

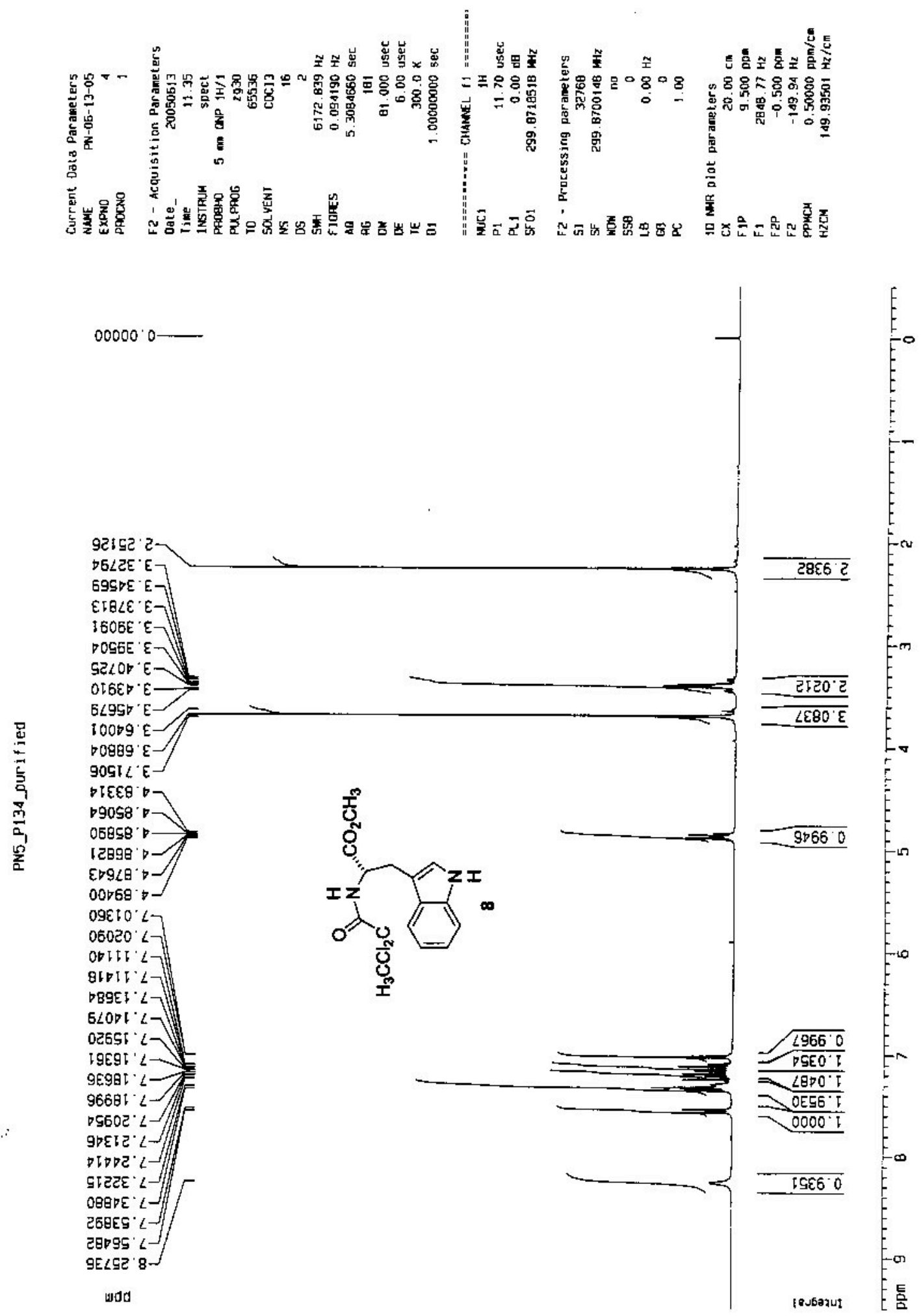

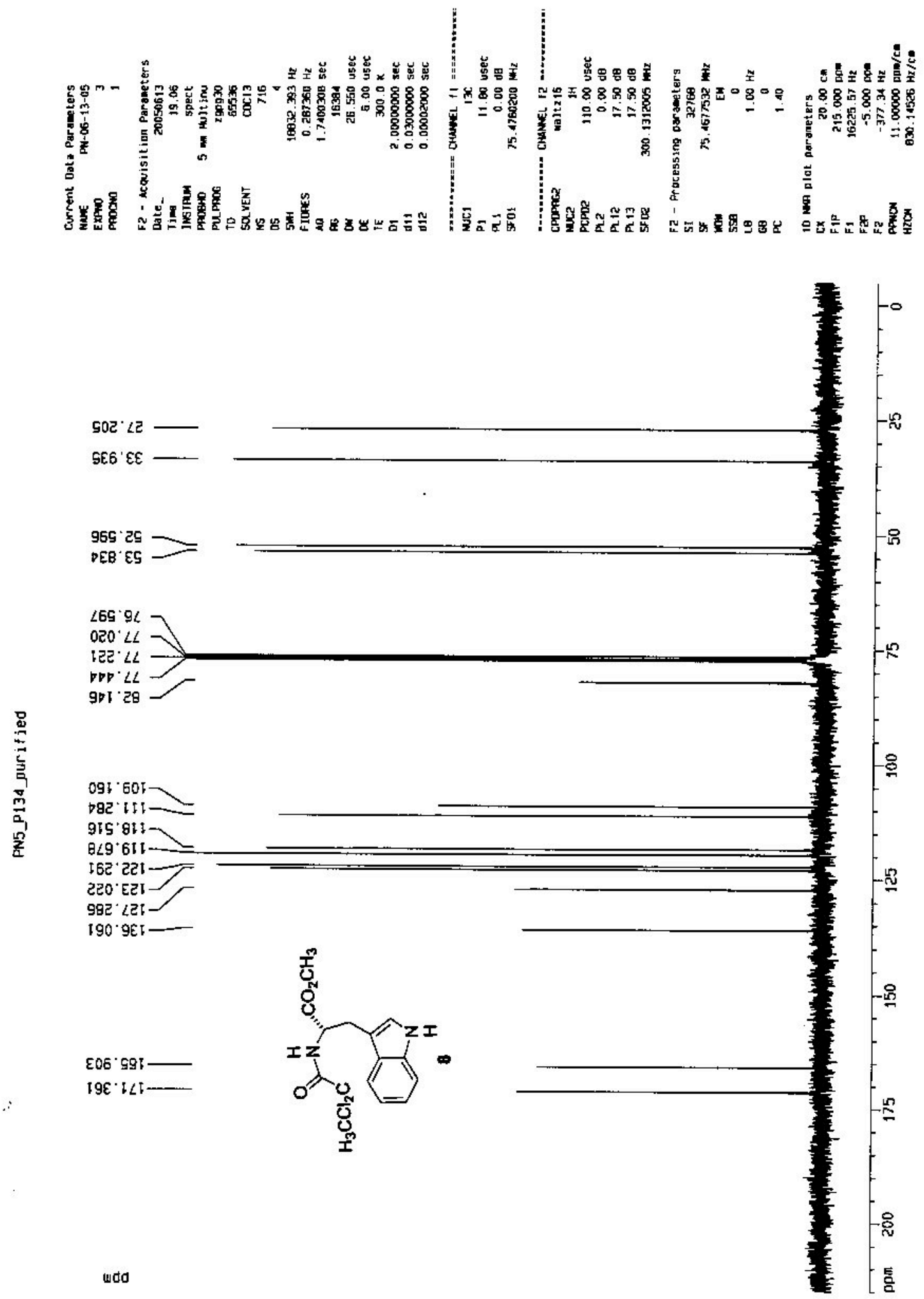

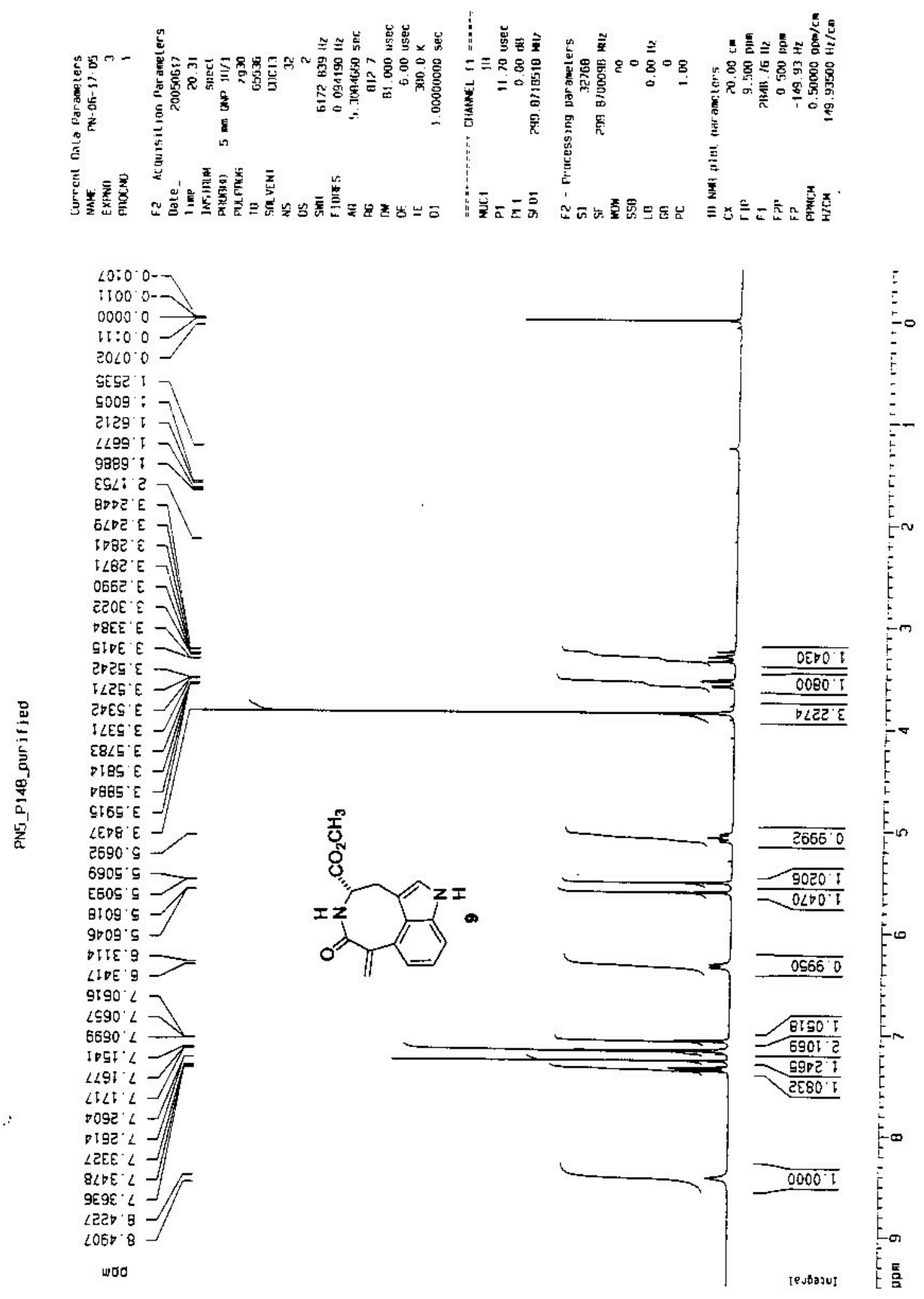


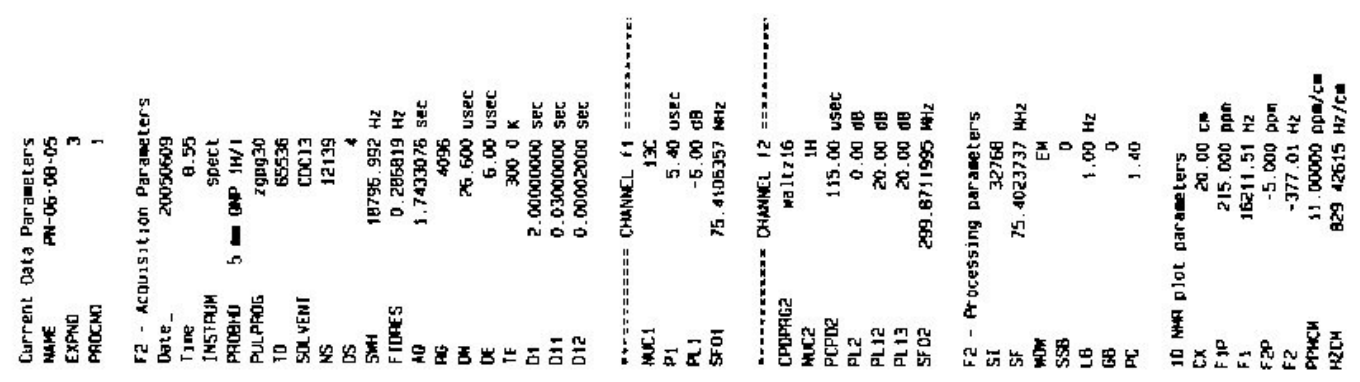

टЕ. $\varepsilon \varepsilon-$

9.6. 29

टl $L \mathrm{SS}$

B19.9L

$2 \mathrm{PO}^{\circ} L$

StC $\angle L-$

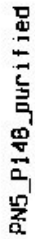

G9E 0 St-

हटटर दा -

मEg git

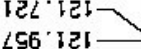

बEब. $551-7$

$\mathrm{SDC}+\mathrm{CL}-$

BSS 9EI-

$\forall G \angle \angle B T-$

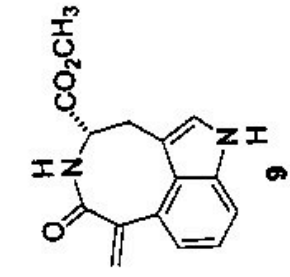

$\angle 69^{\circ} \mathrm{LL}=$

$6 E \angle C \angle 1=$

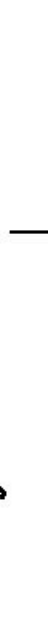



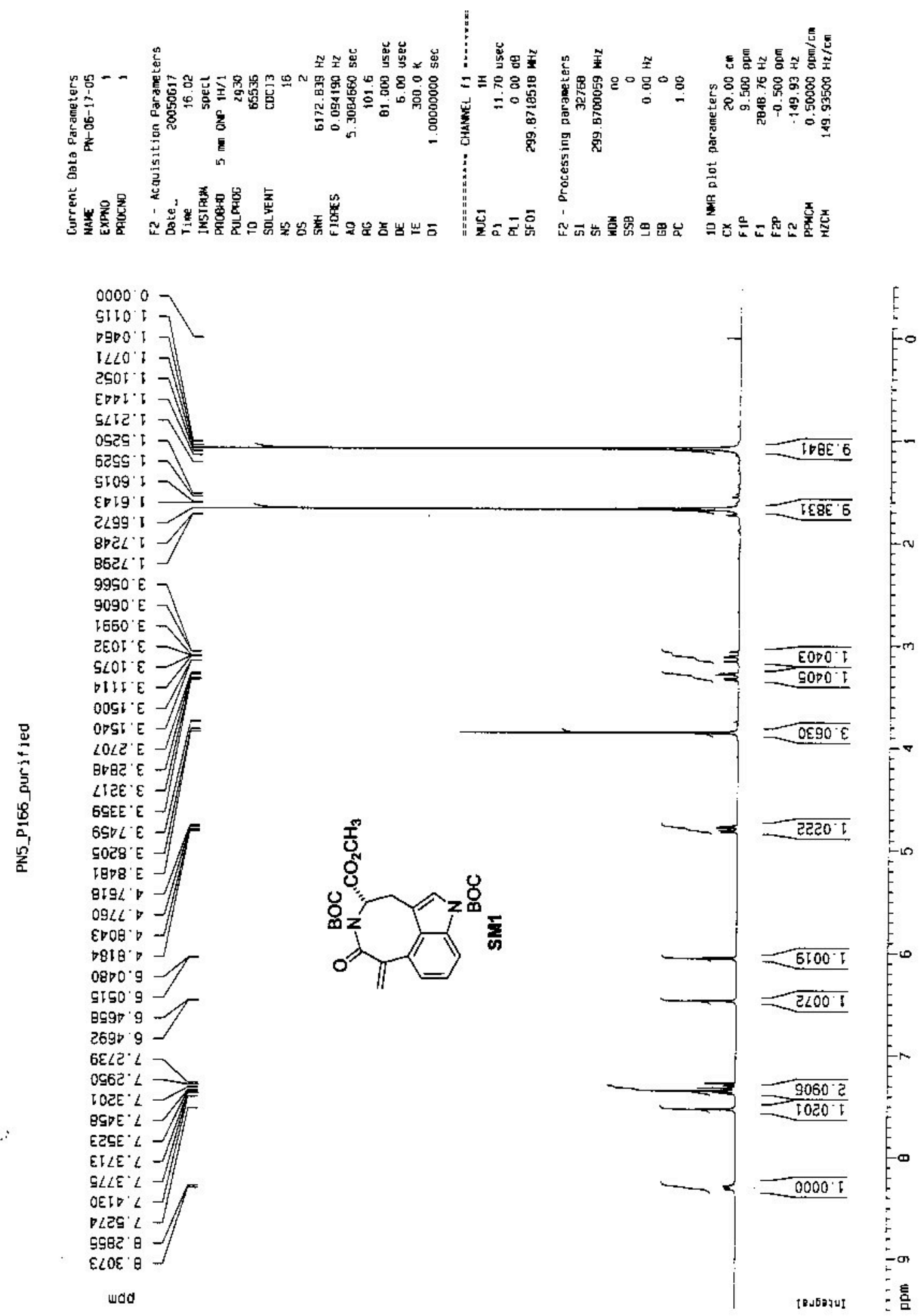

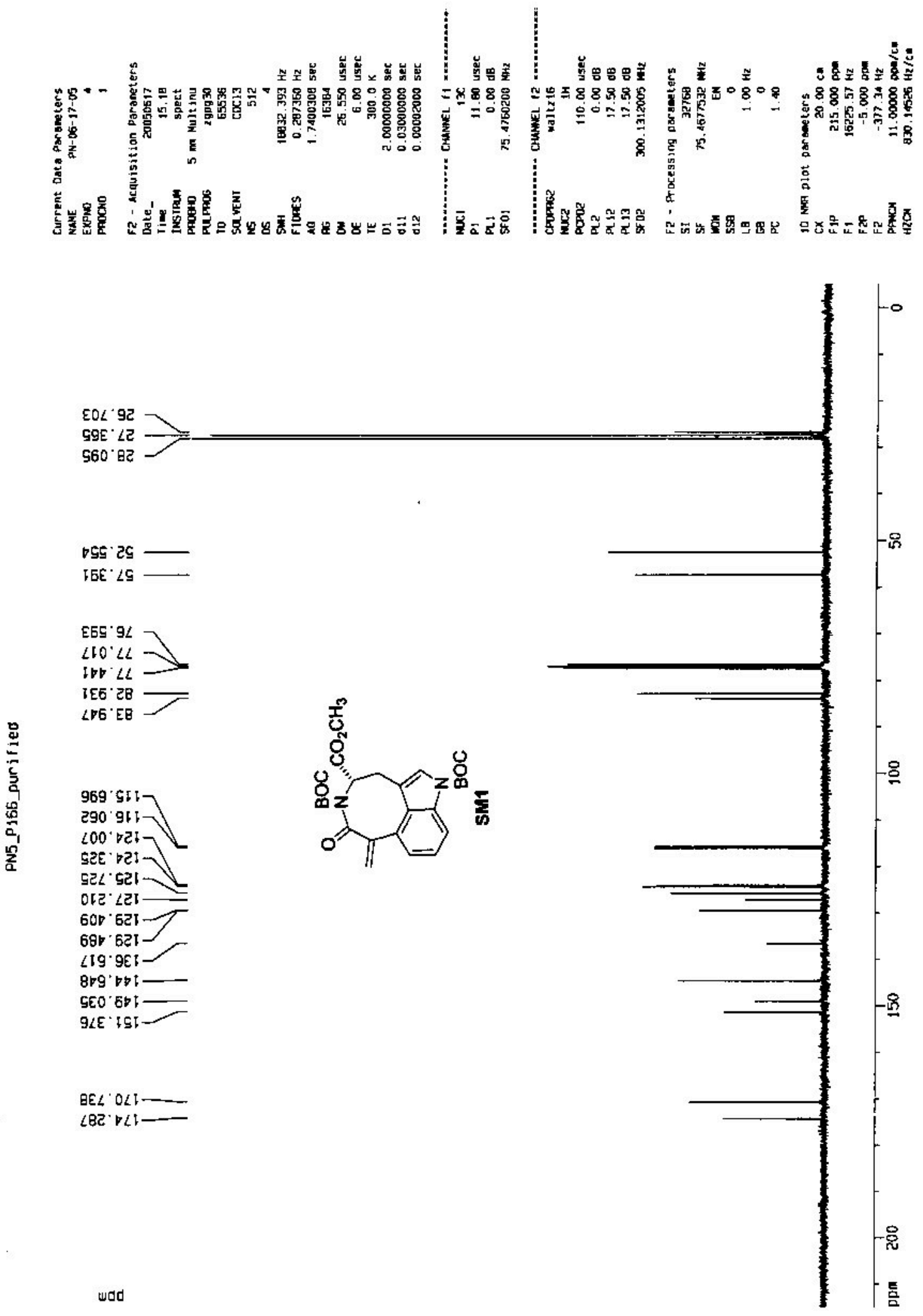

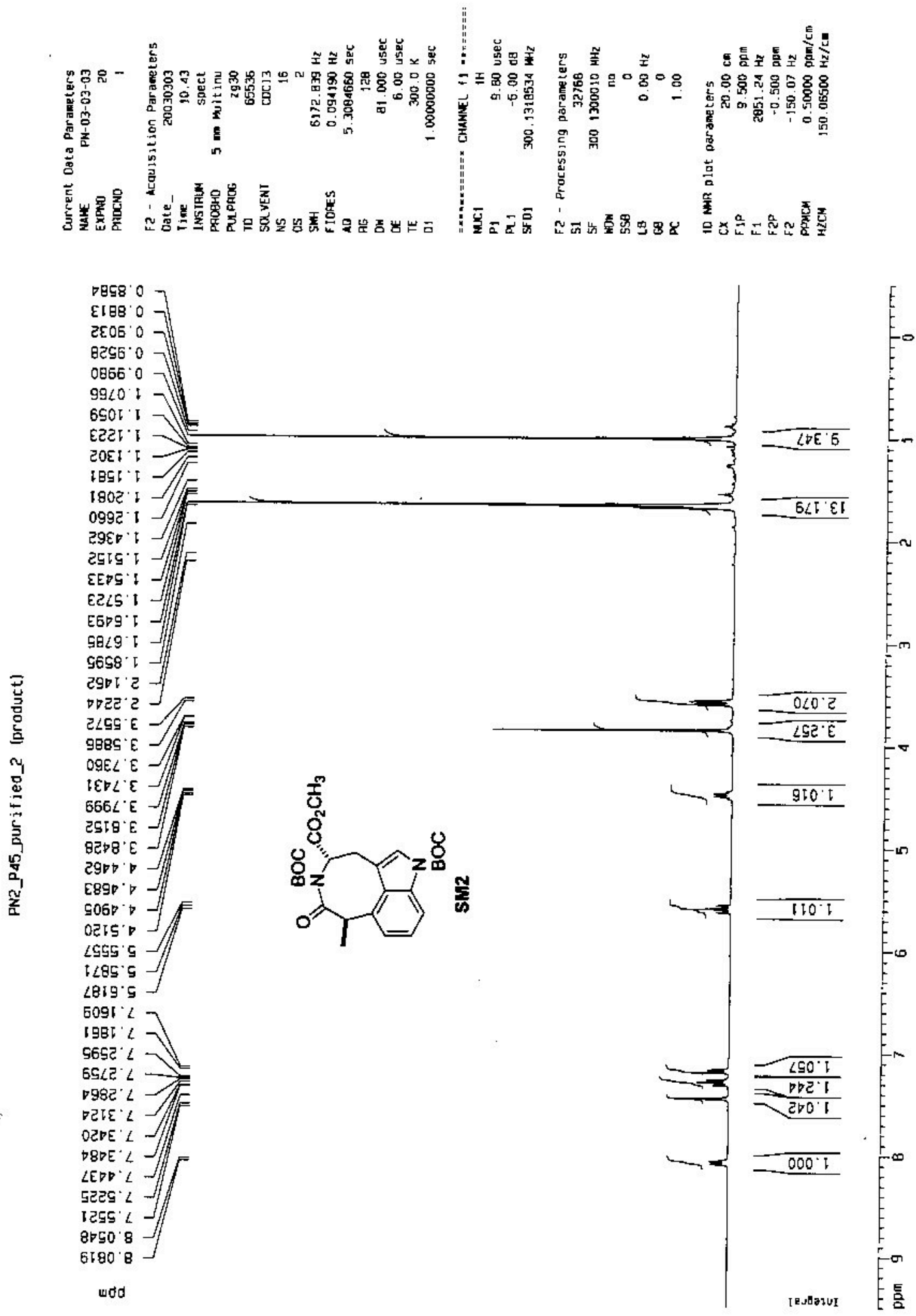

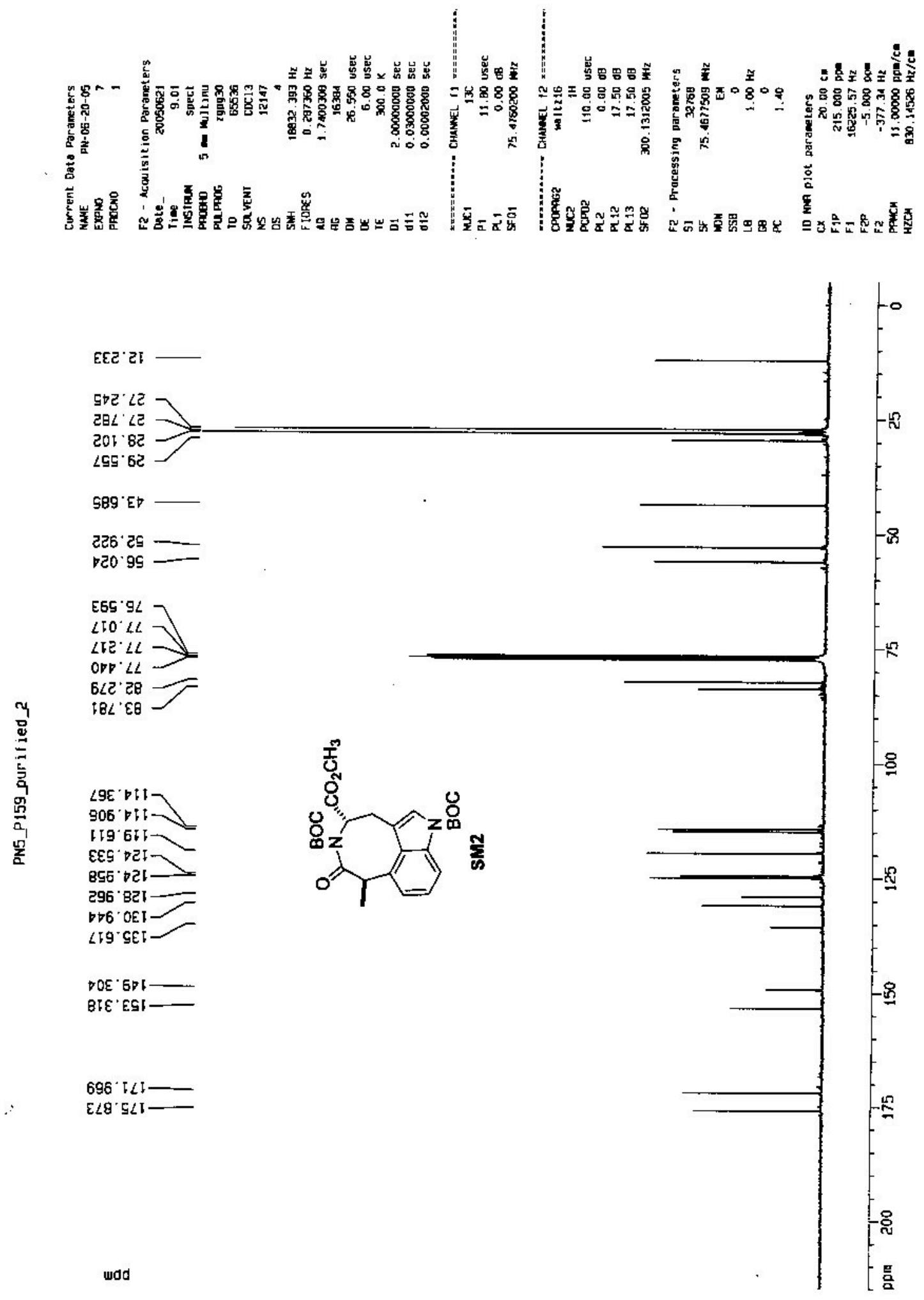

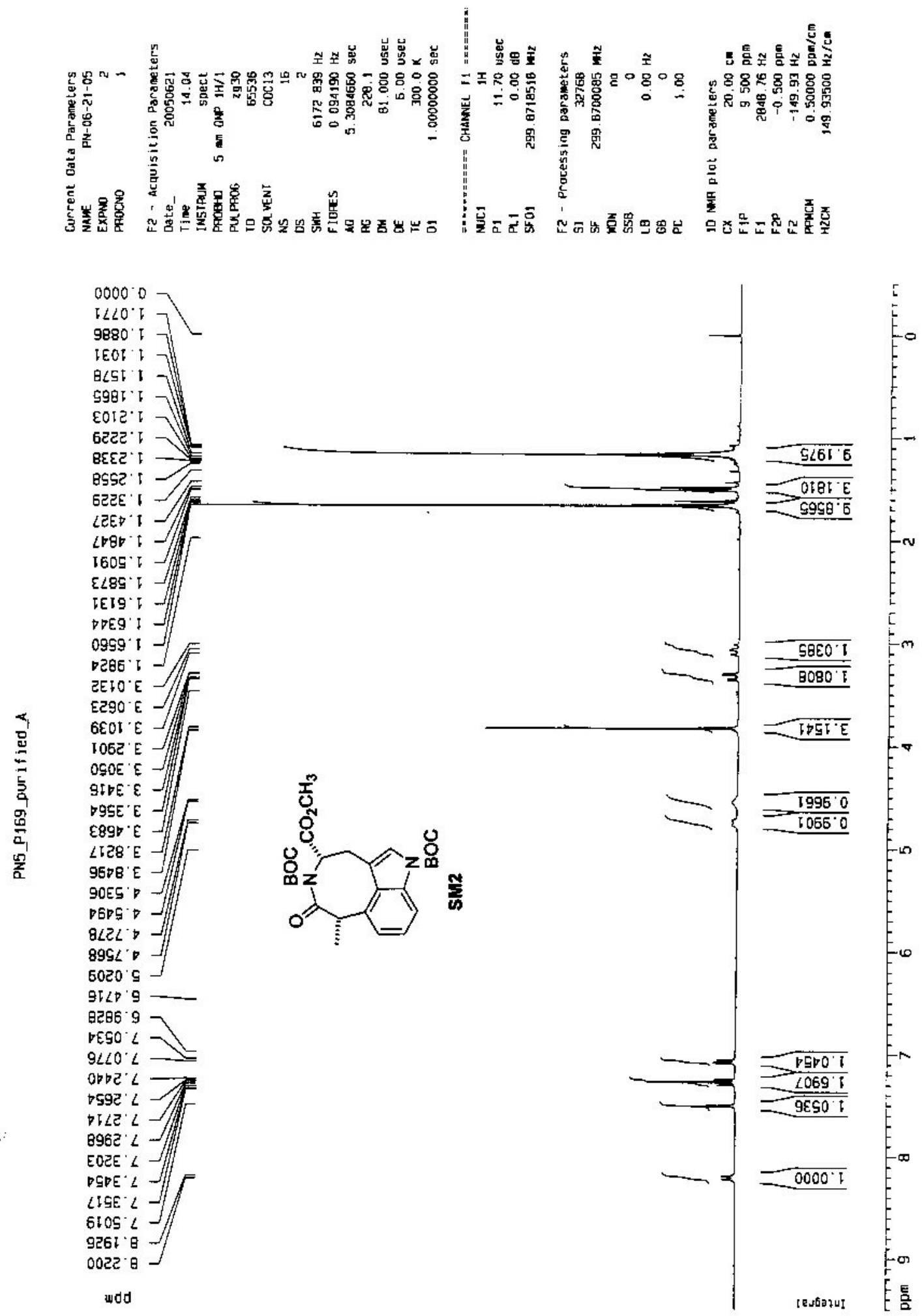

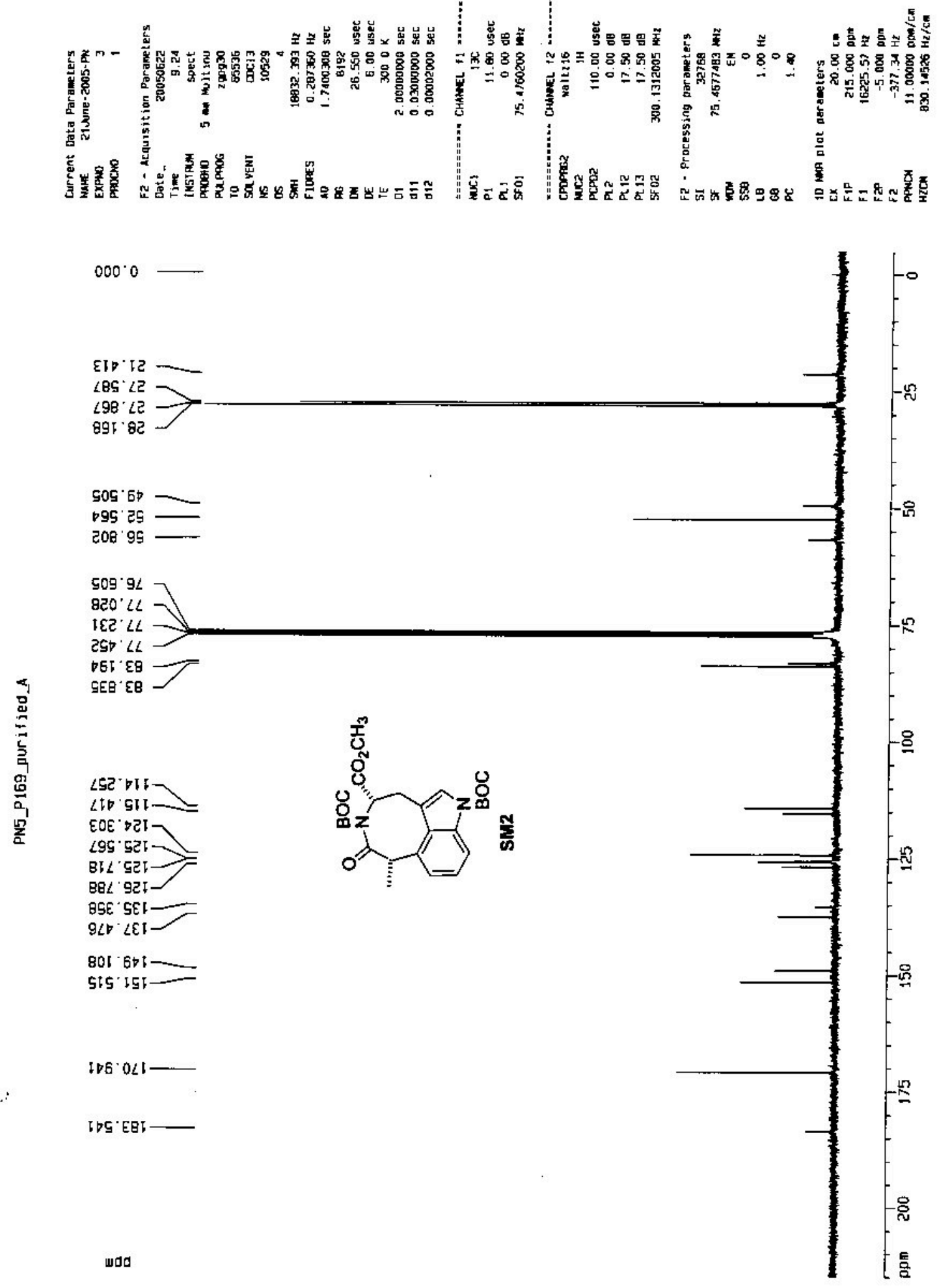

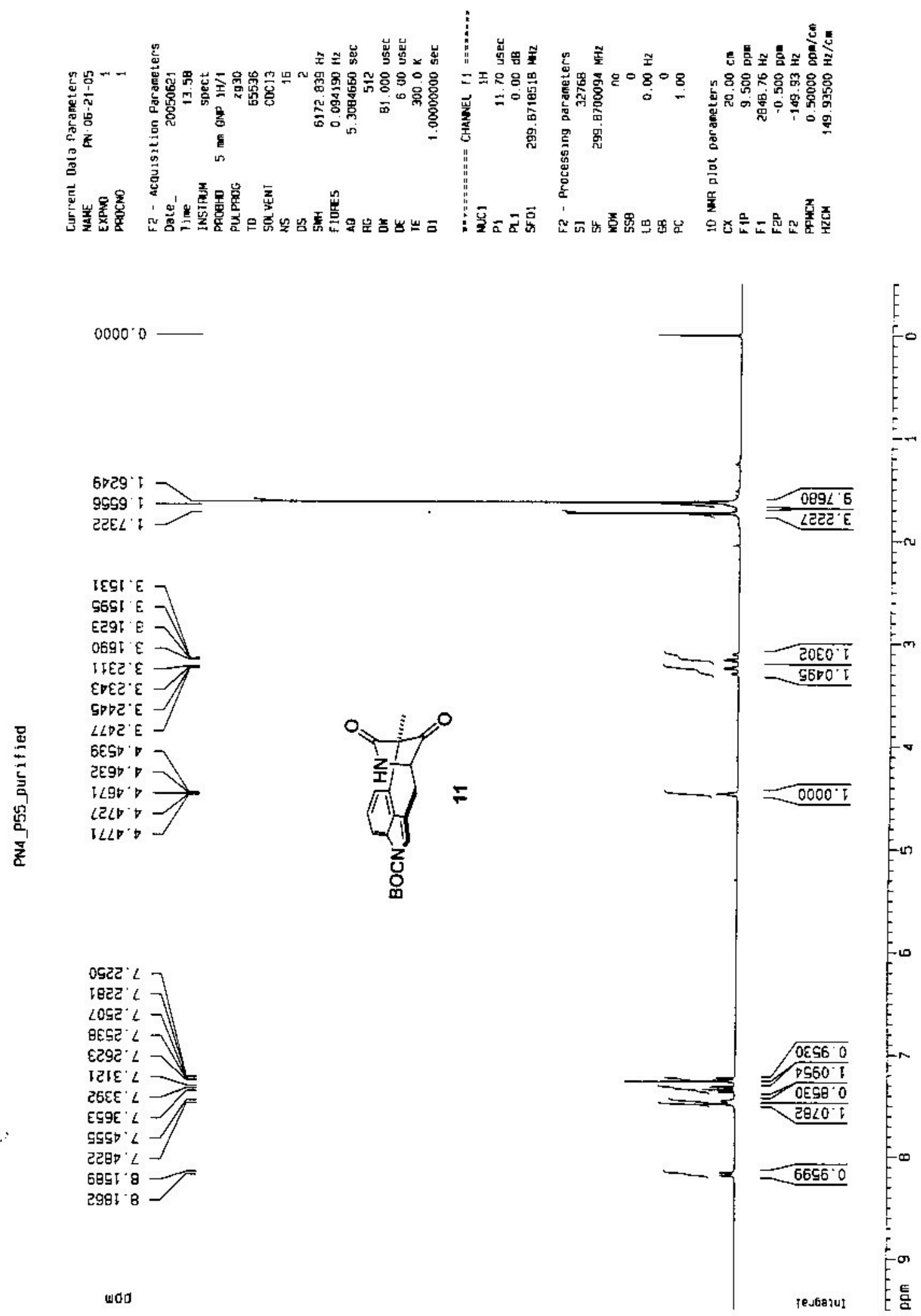

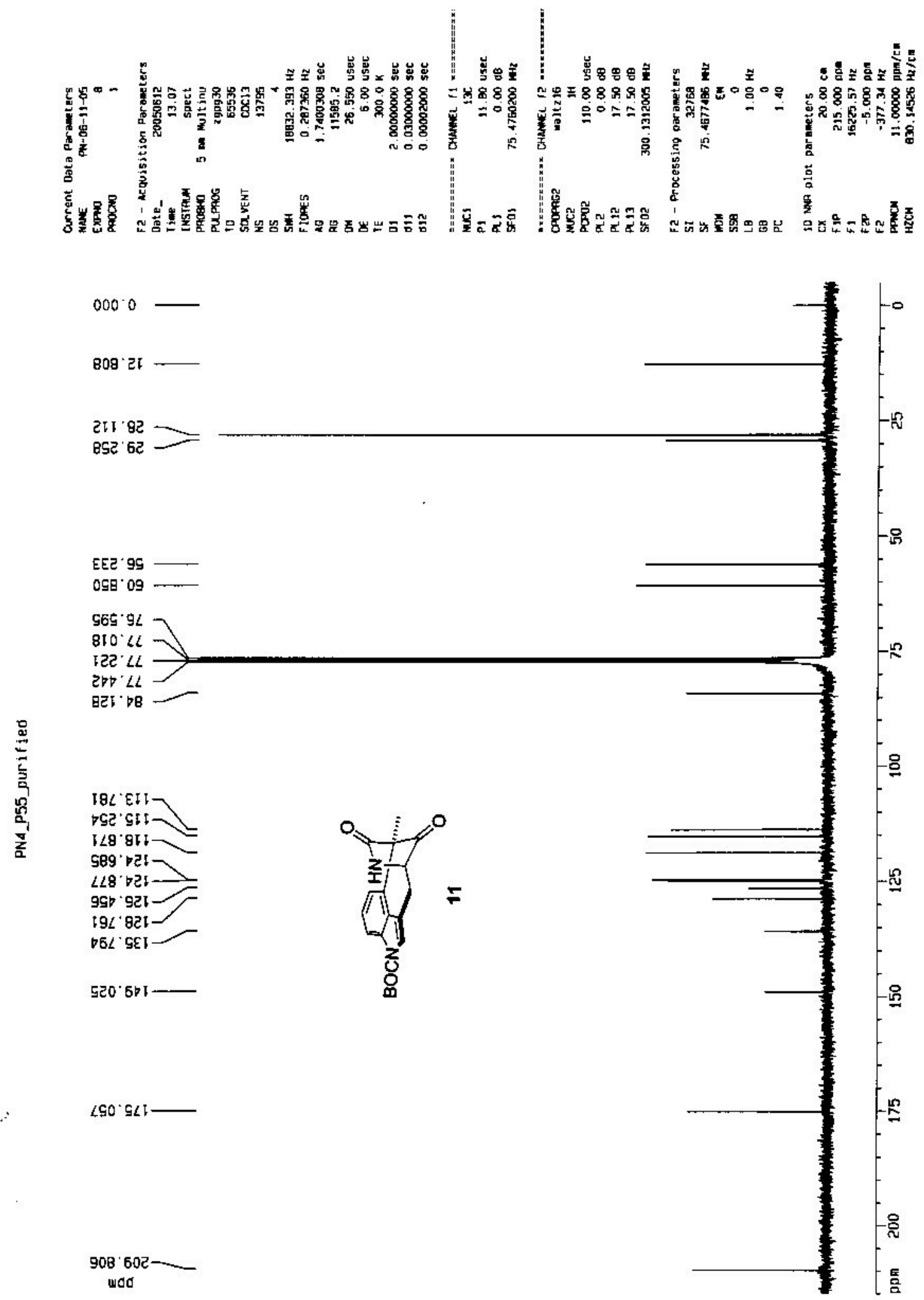

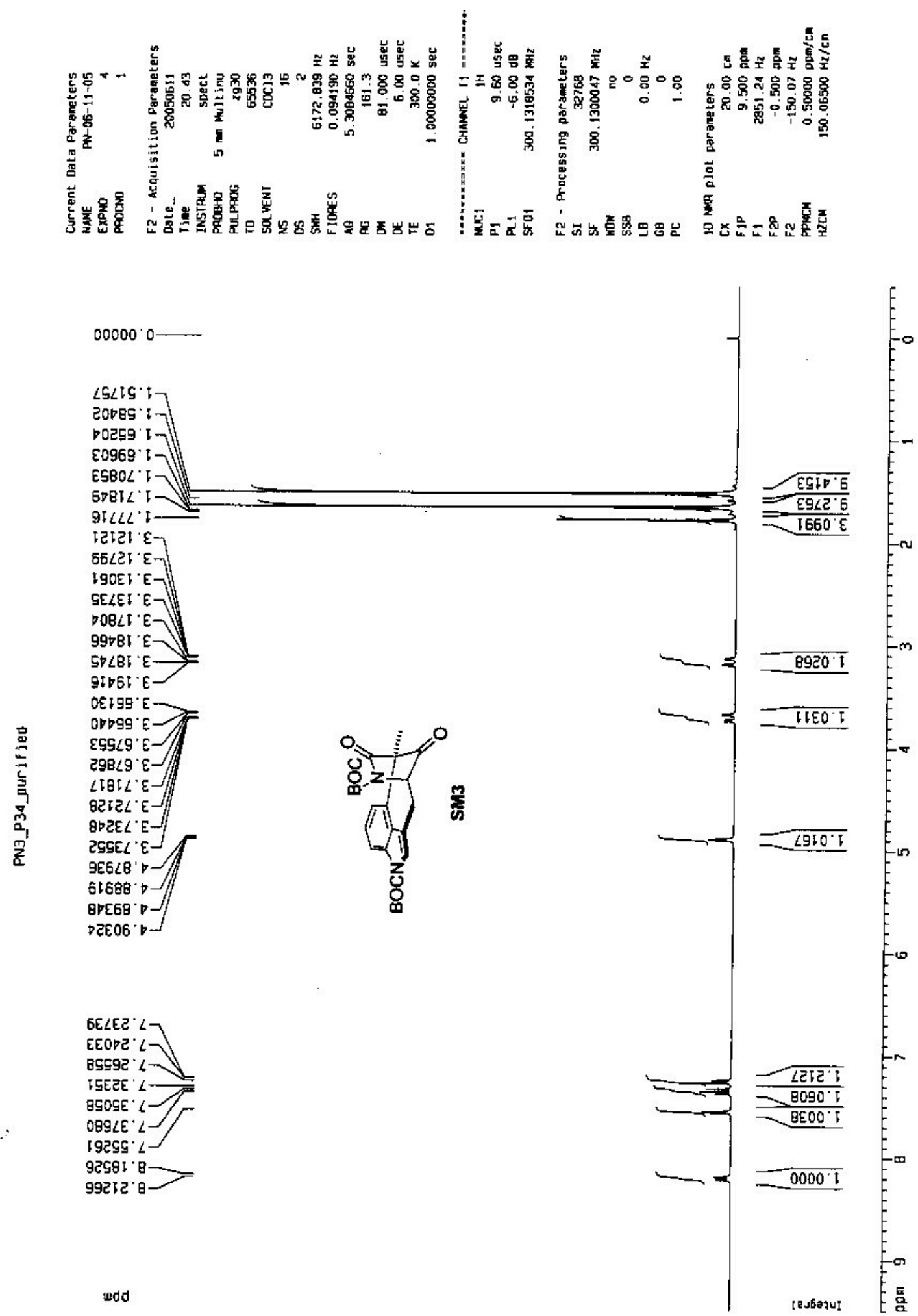

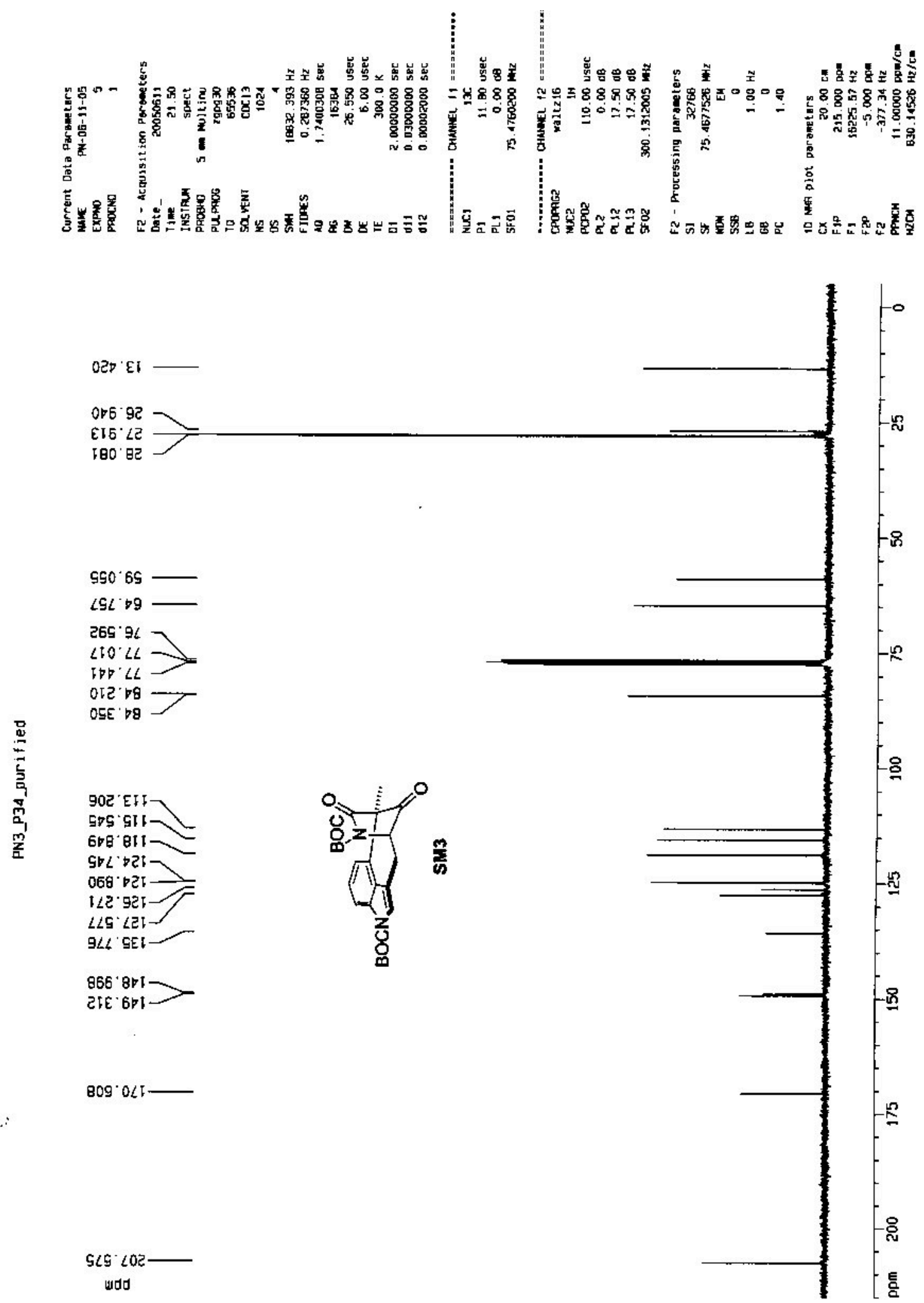

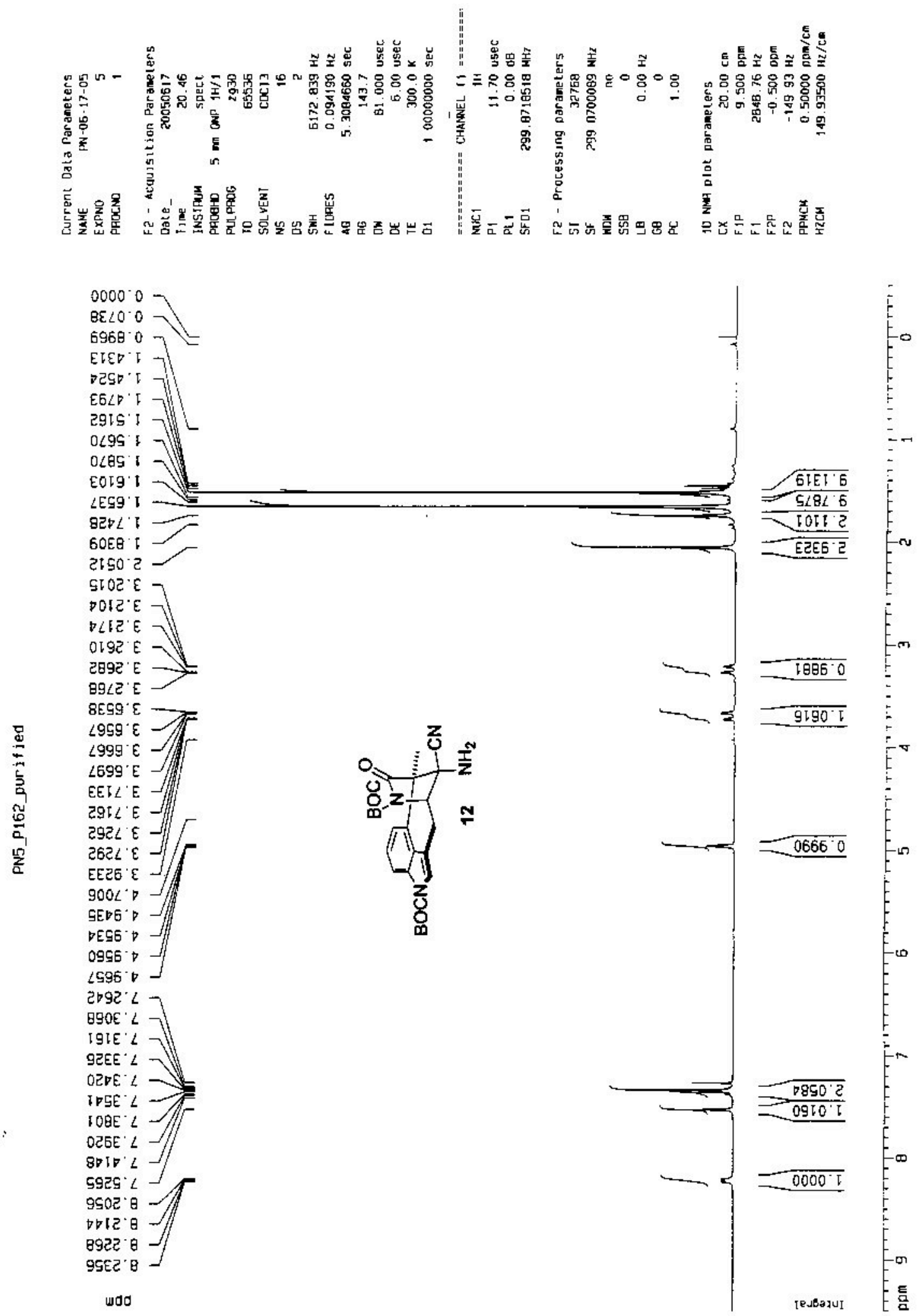

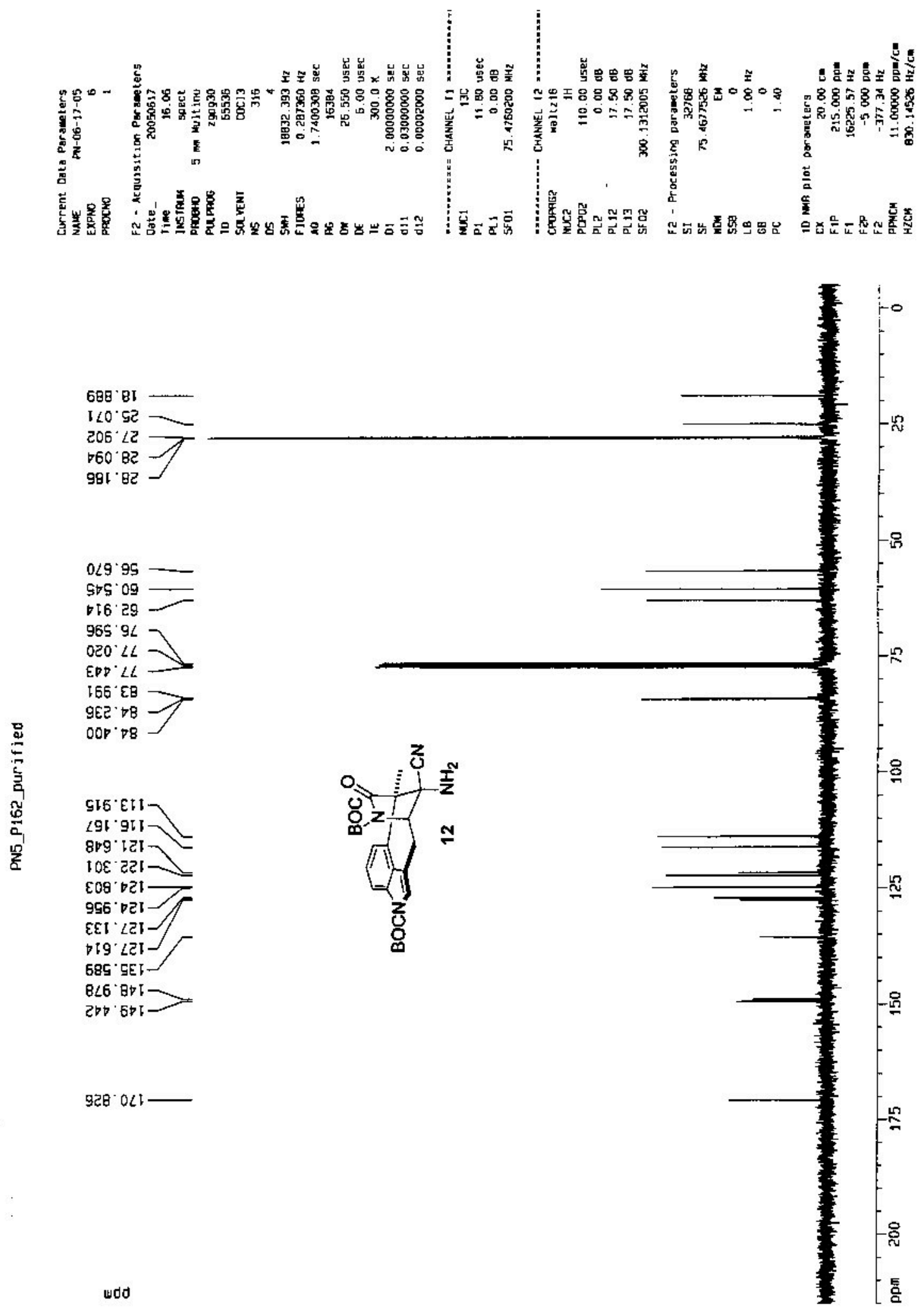

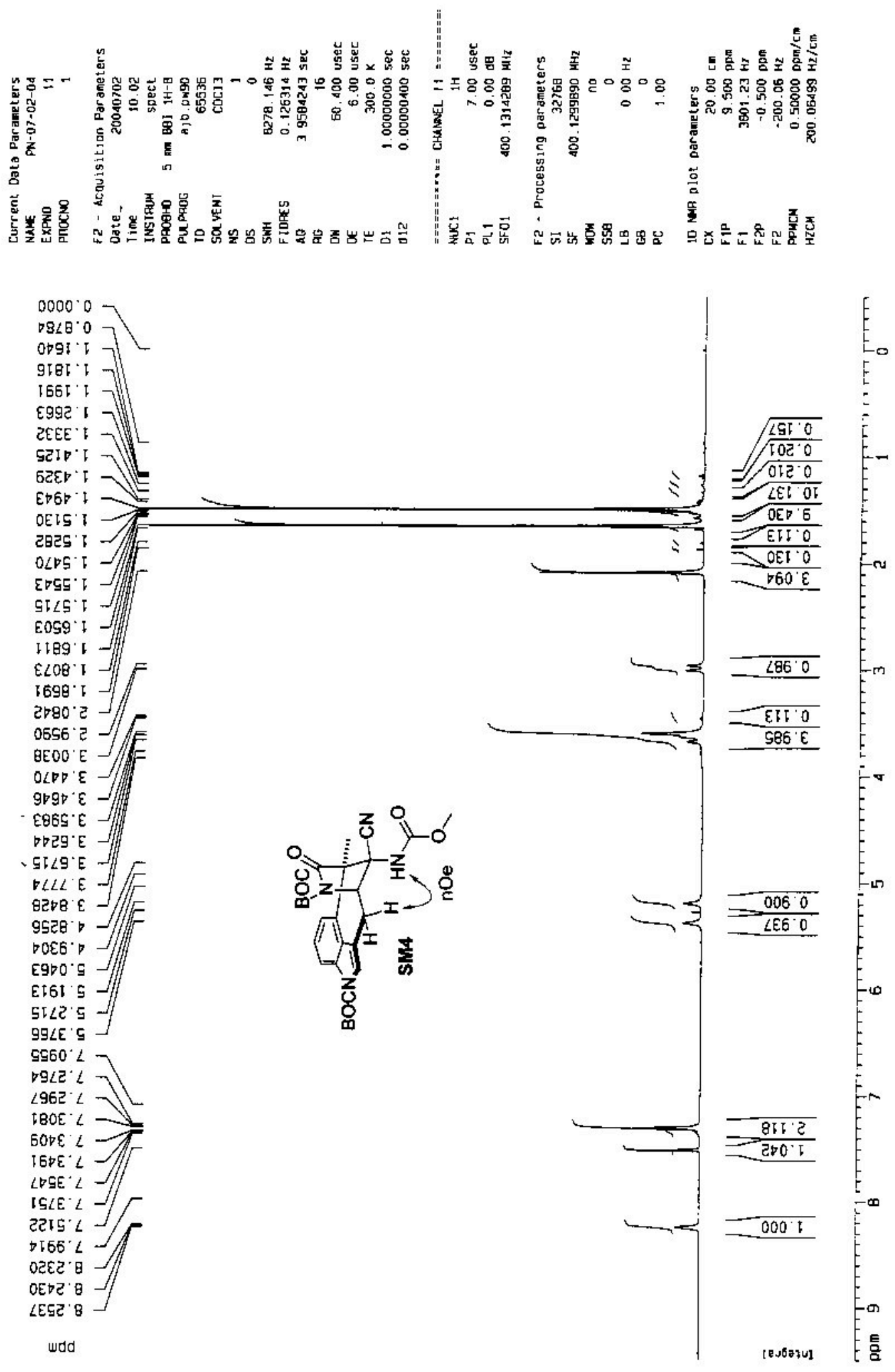

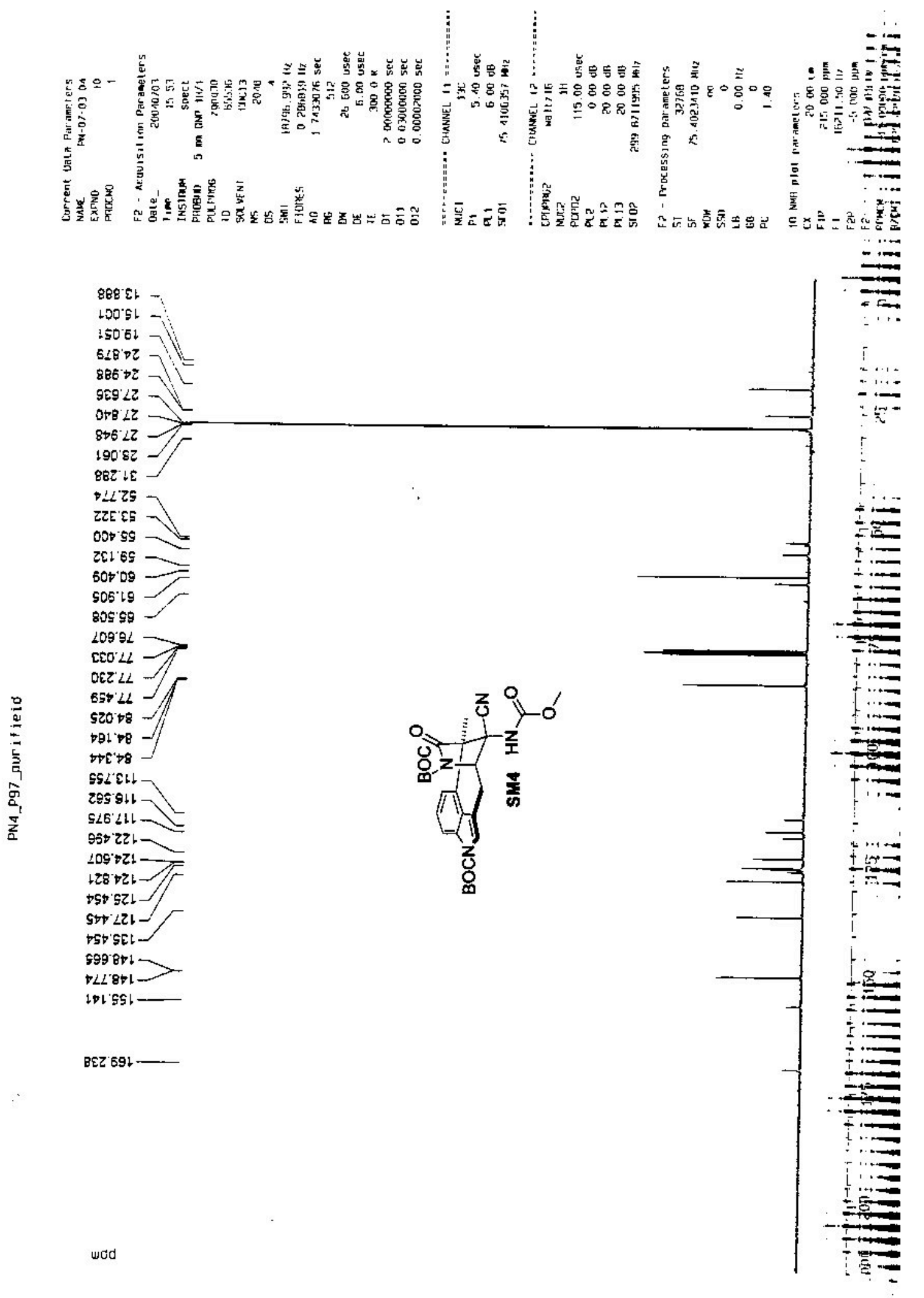

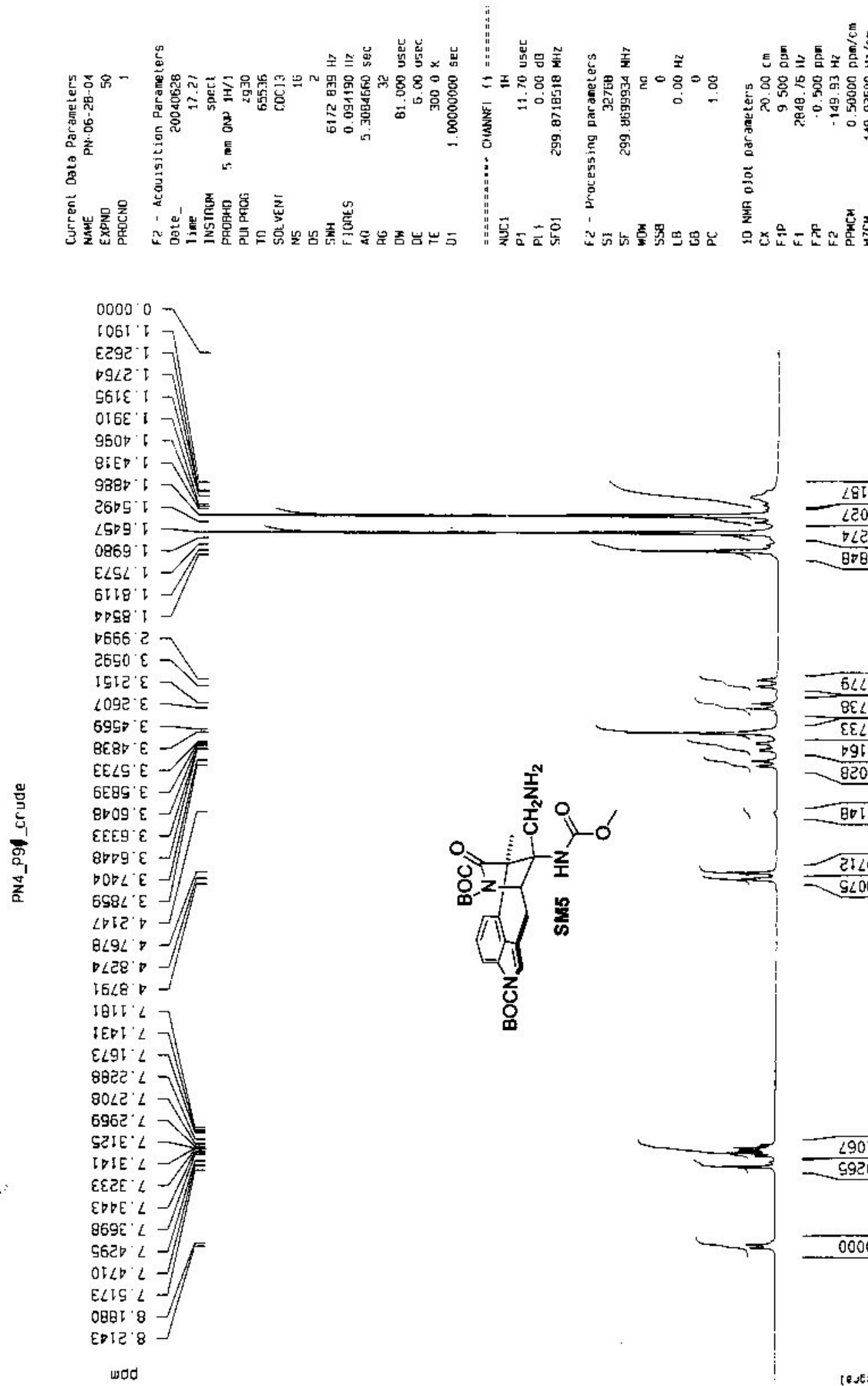

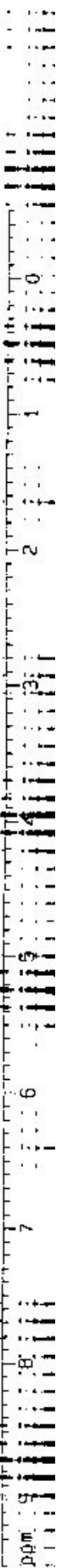

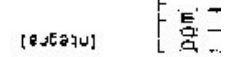



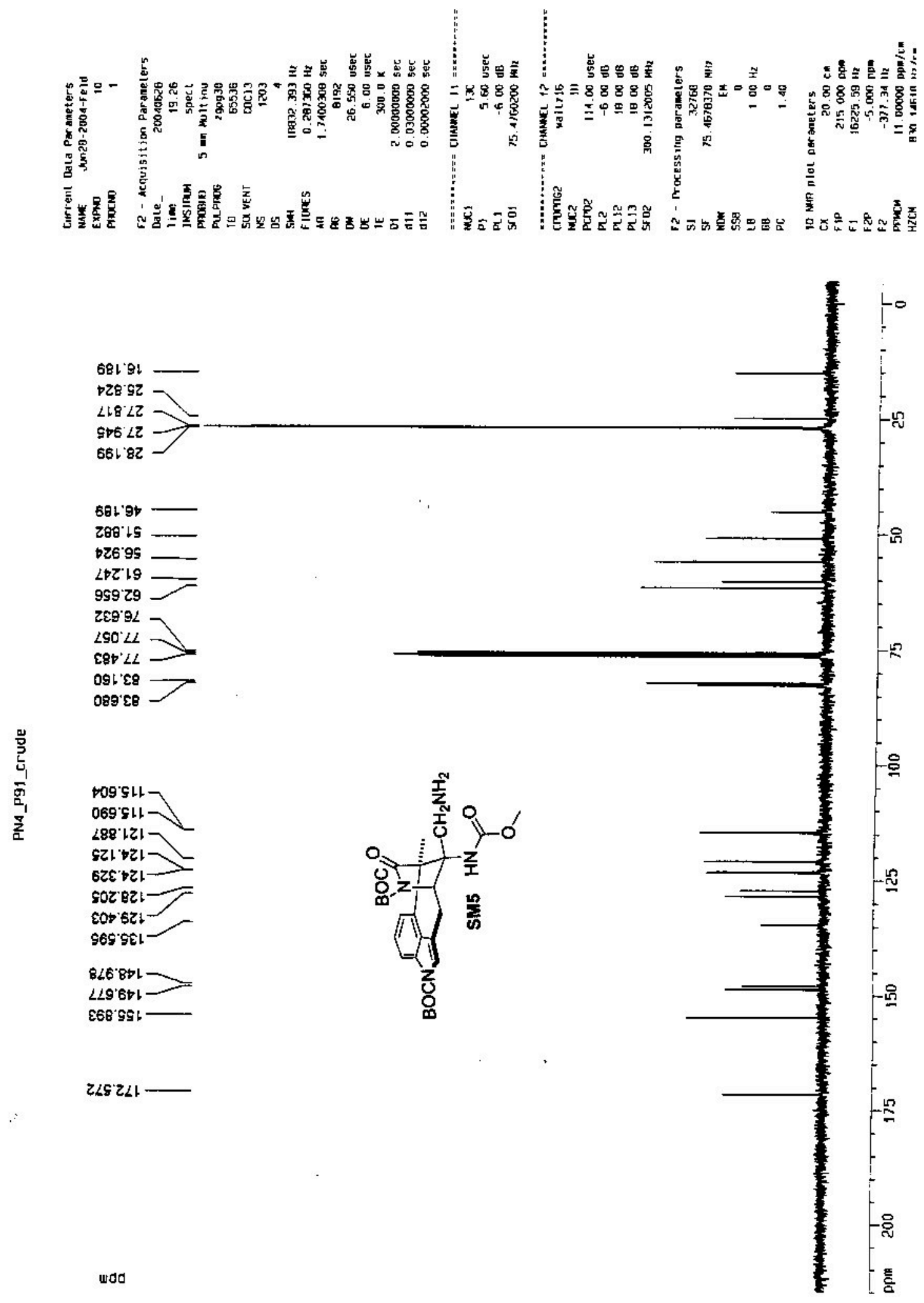

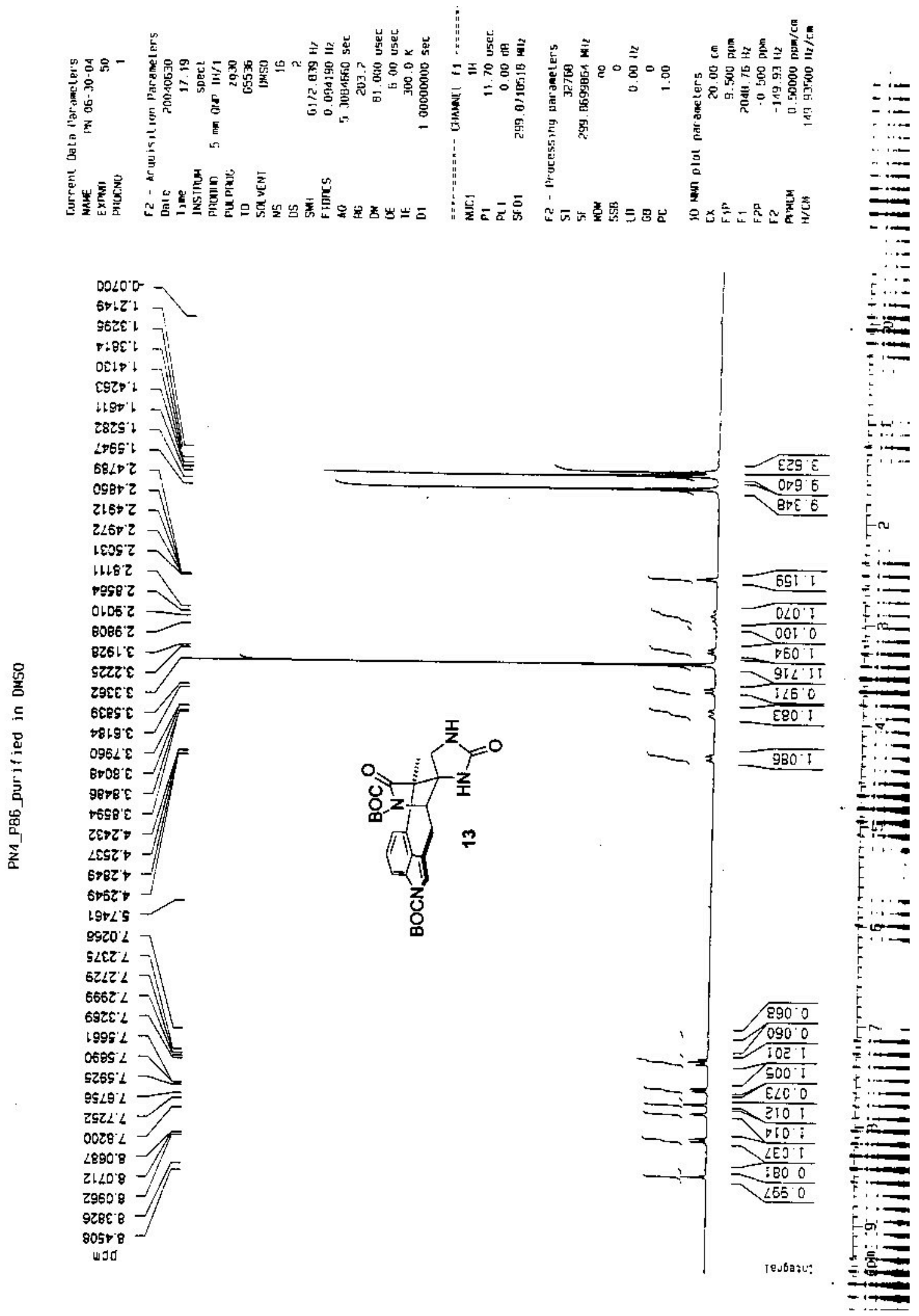


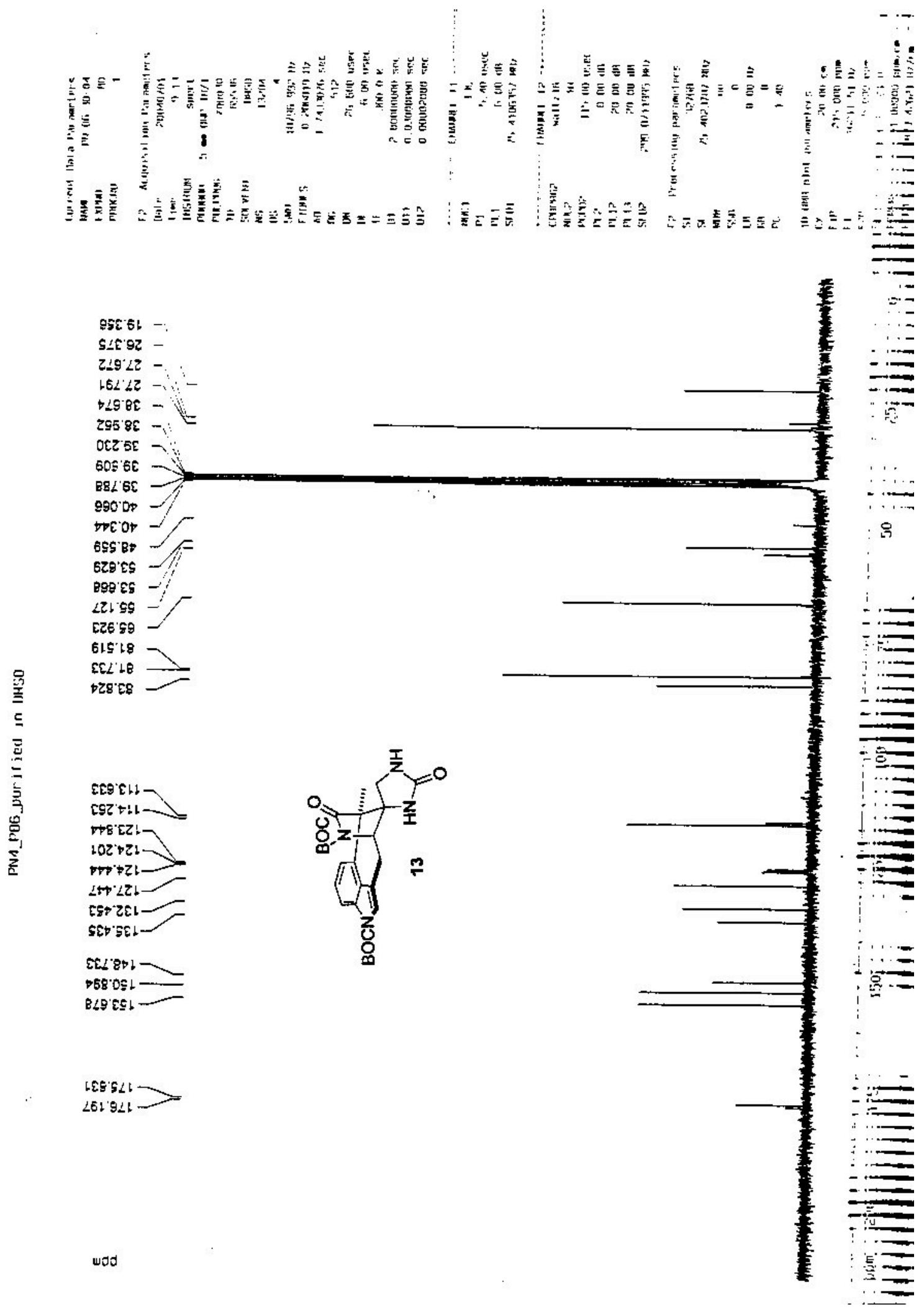



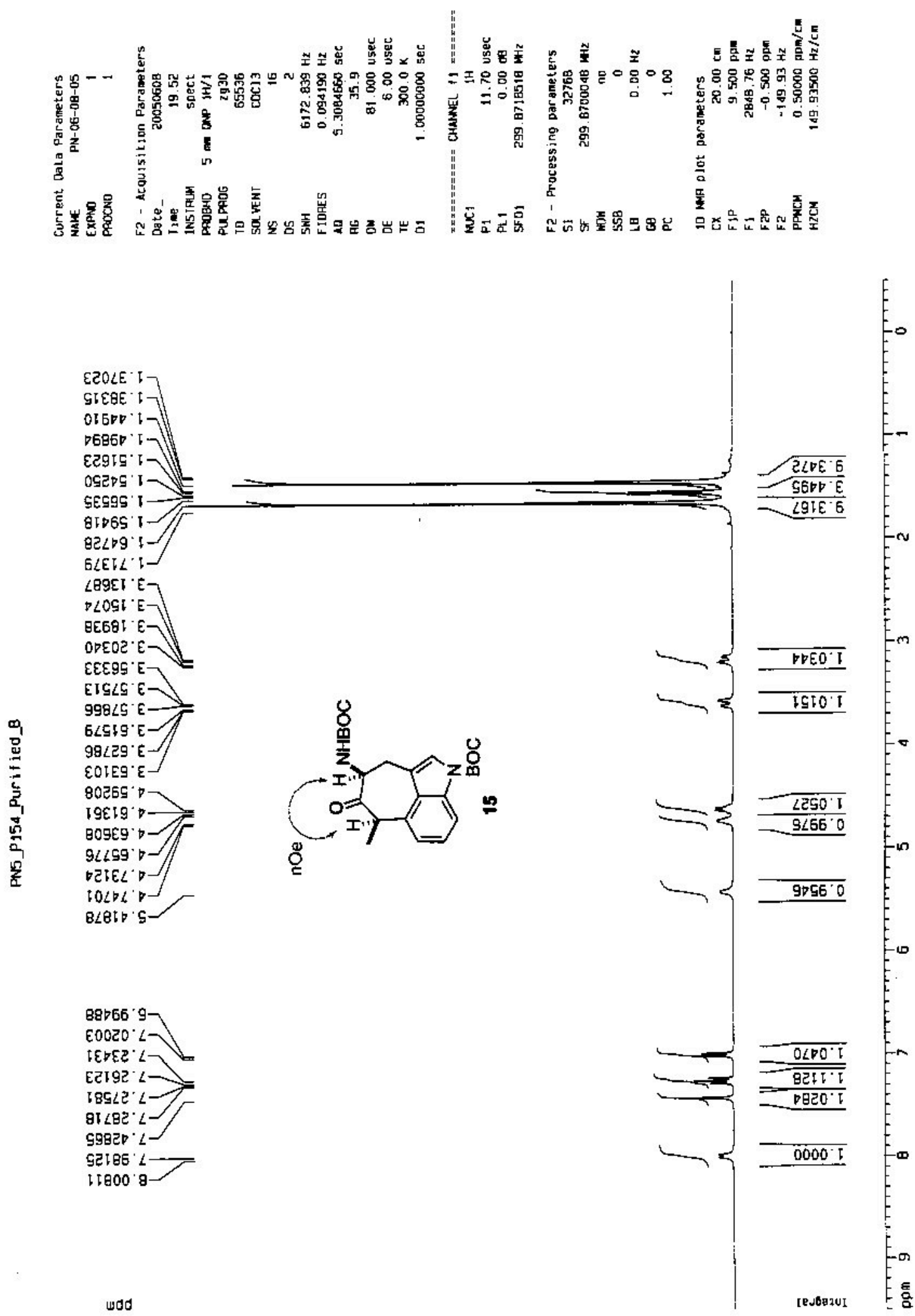

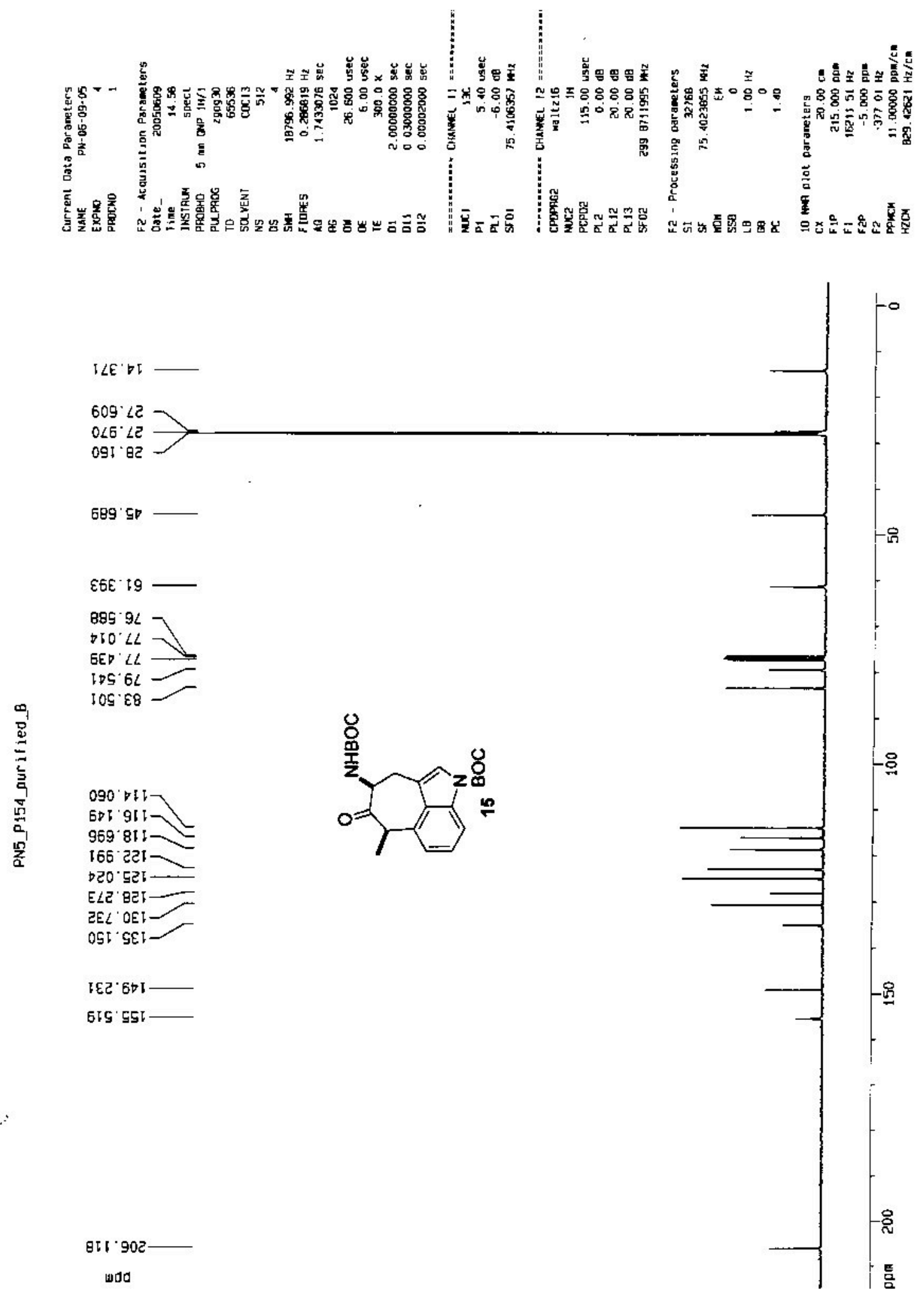

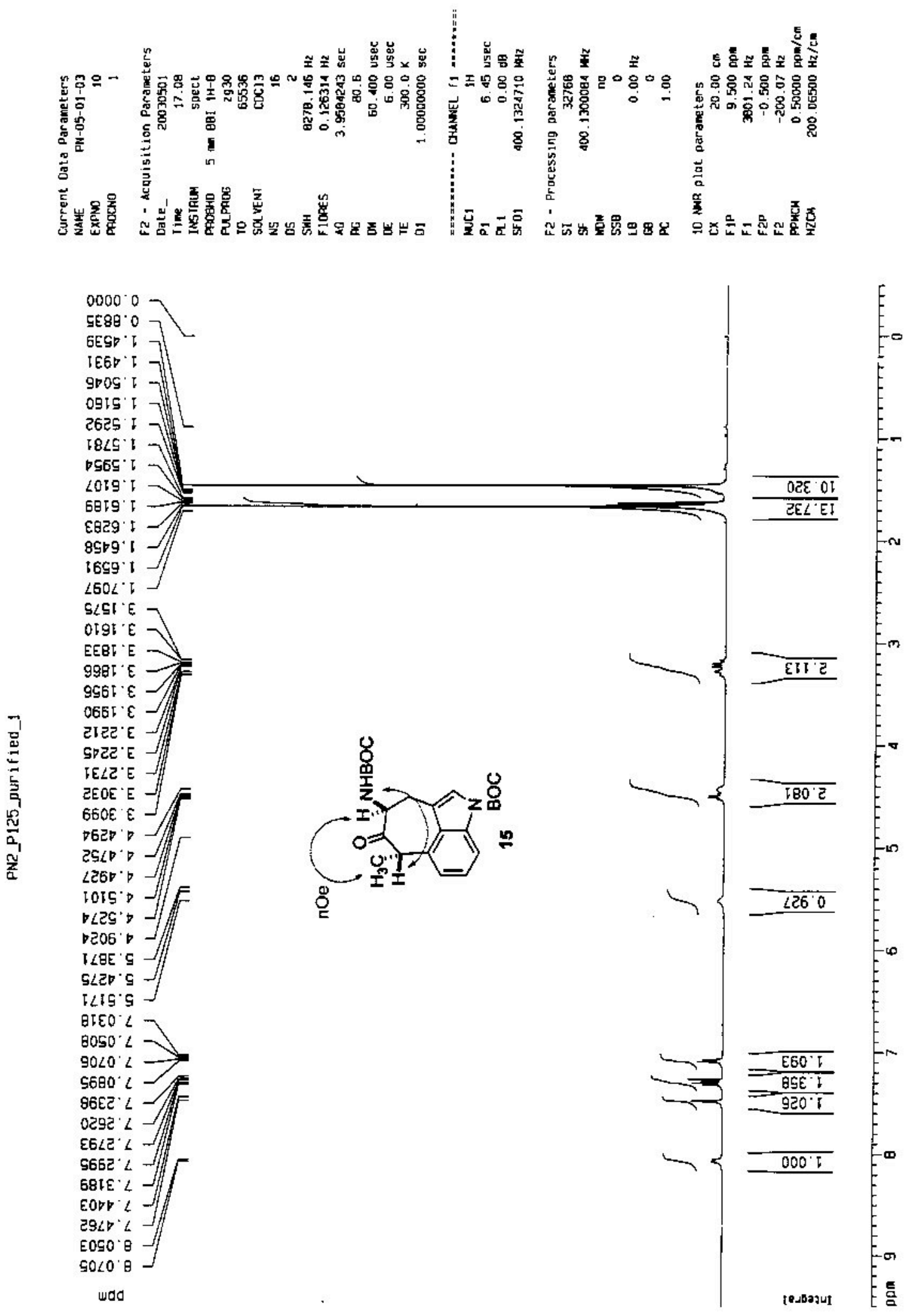

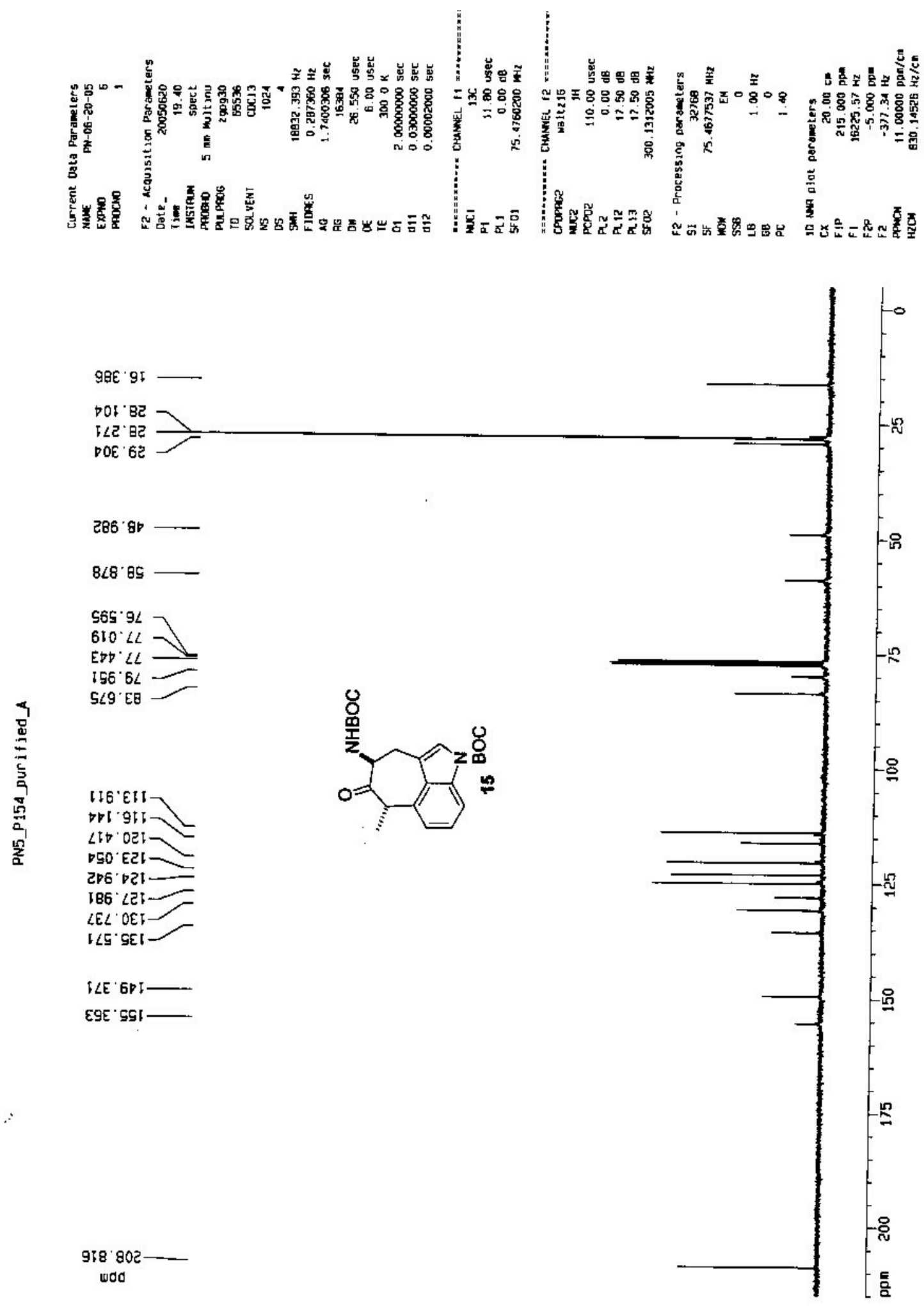

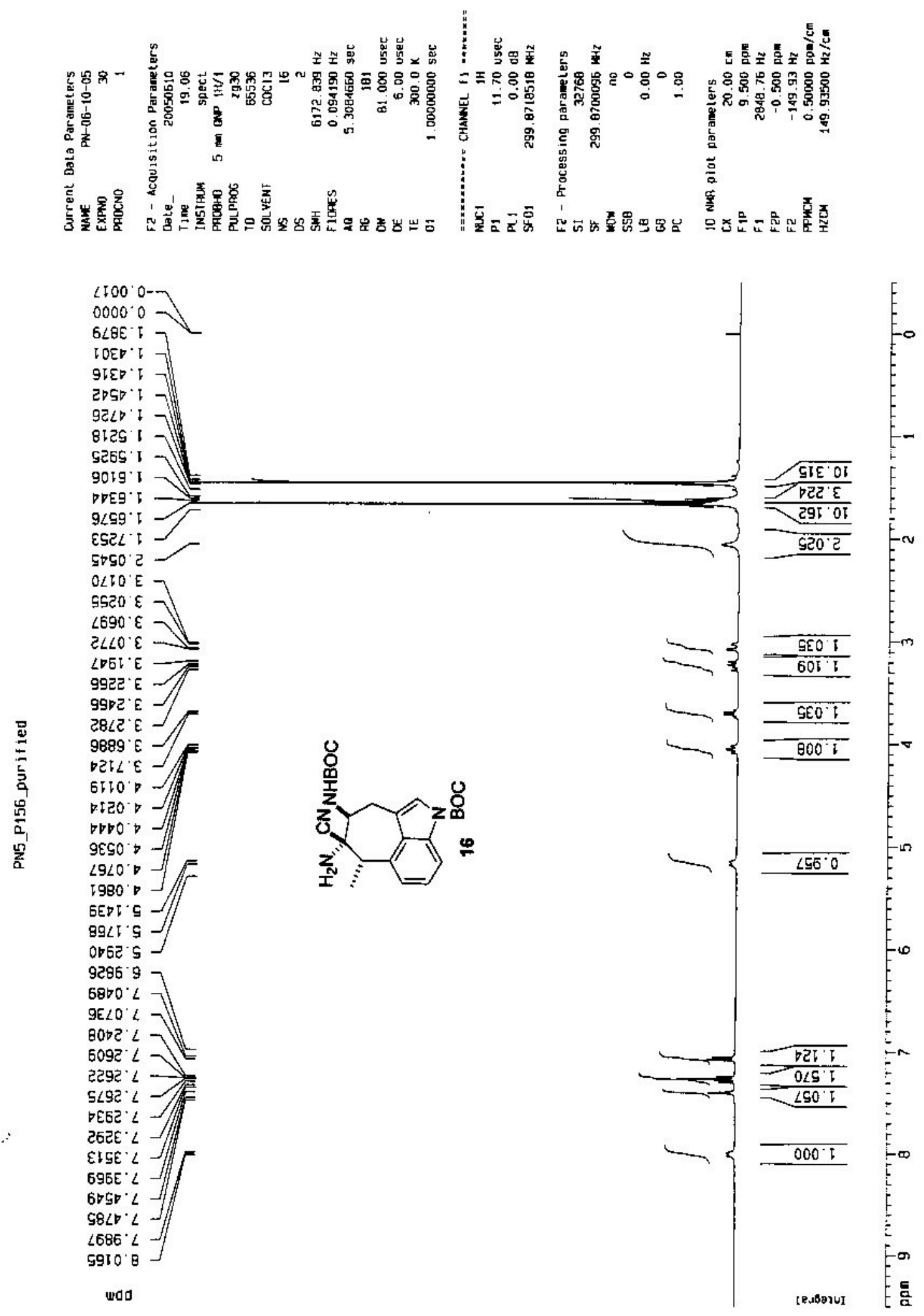

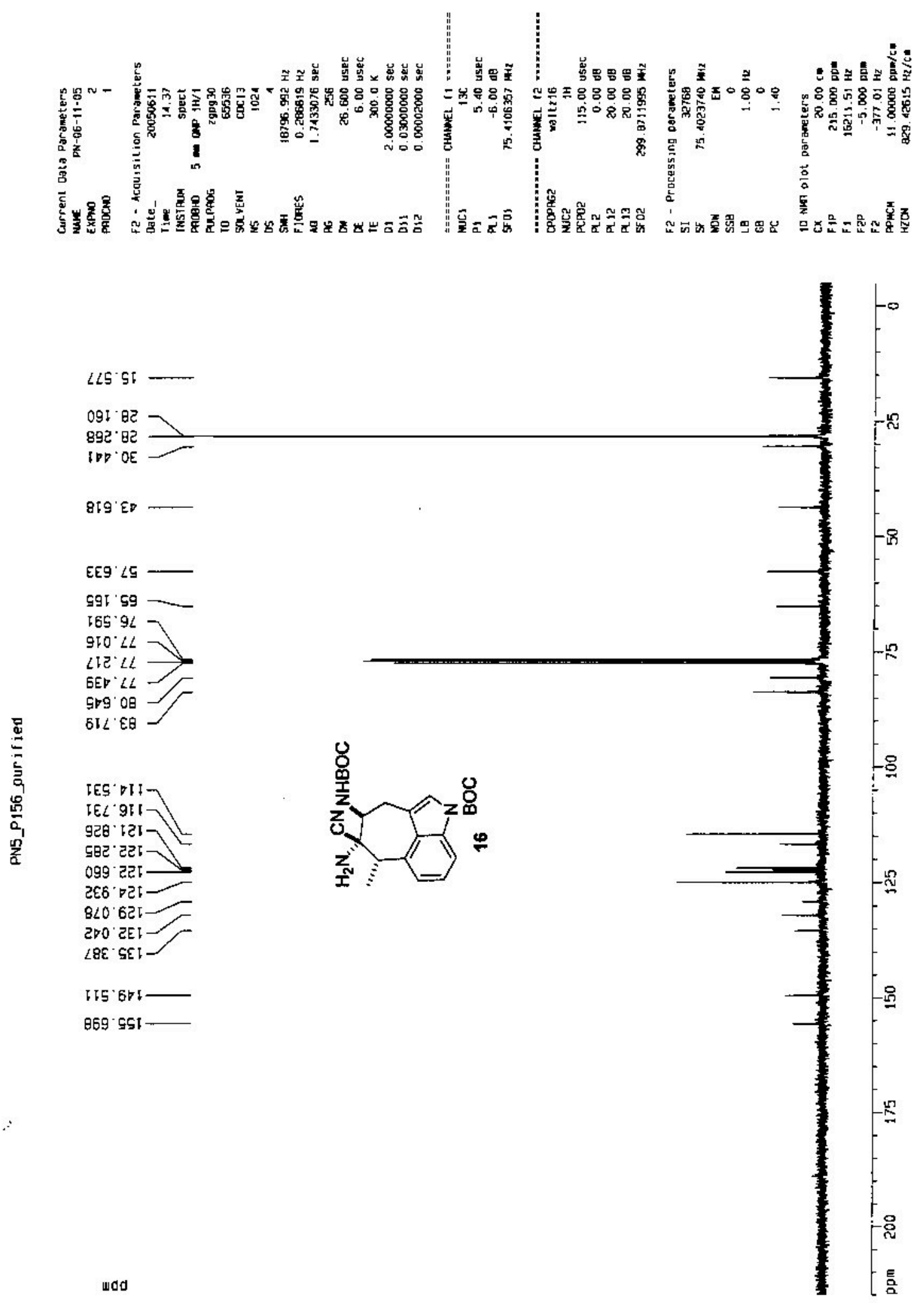

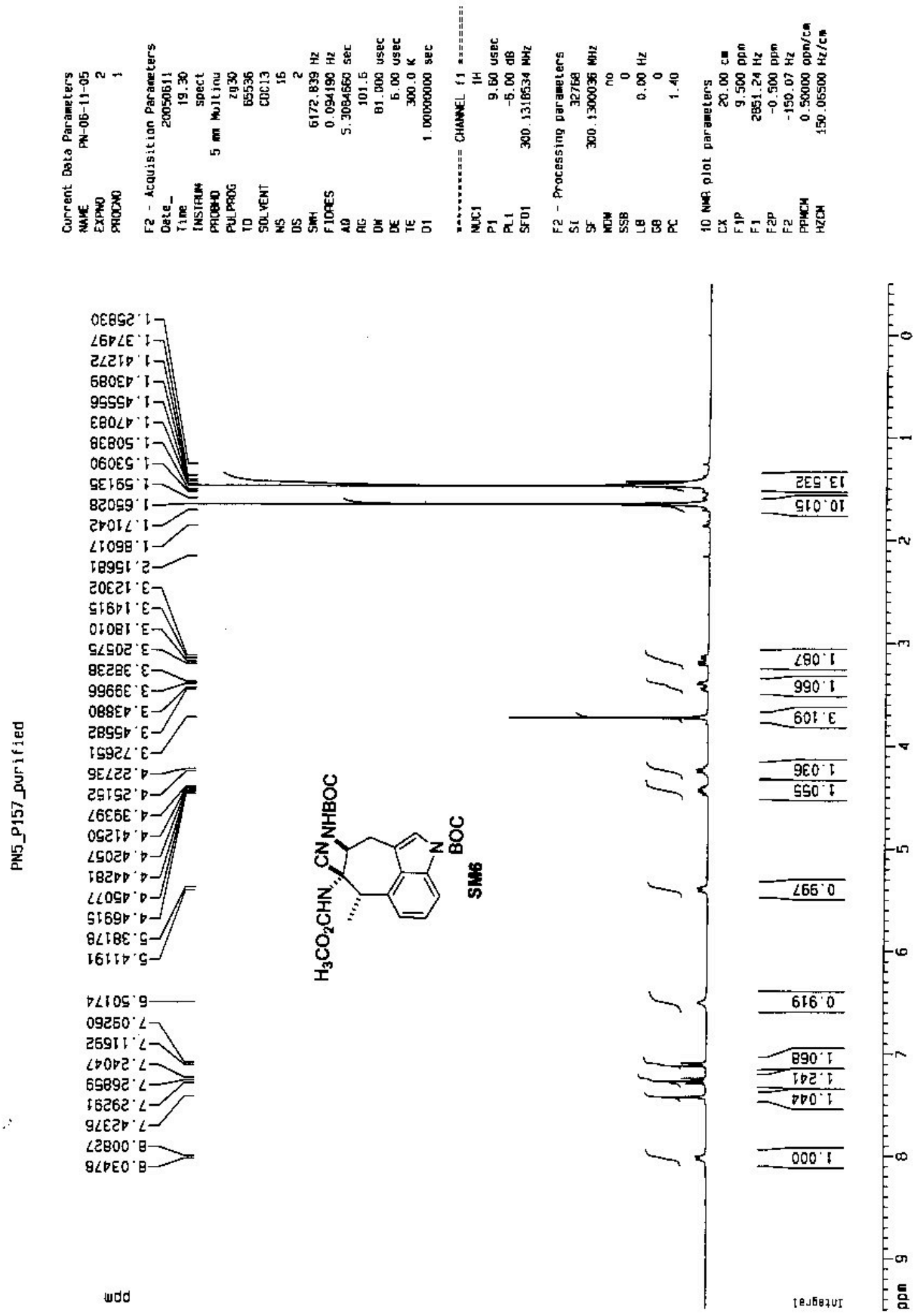

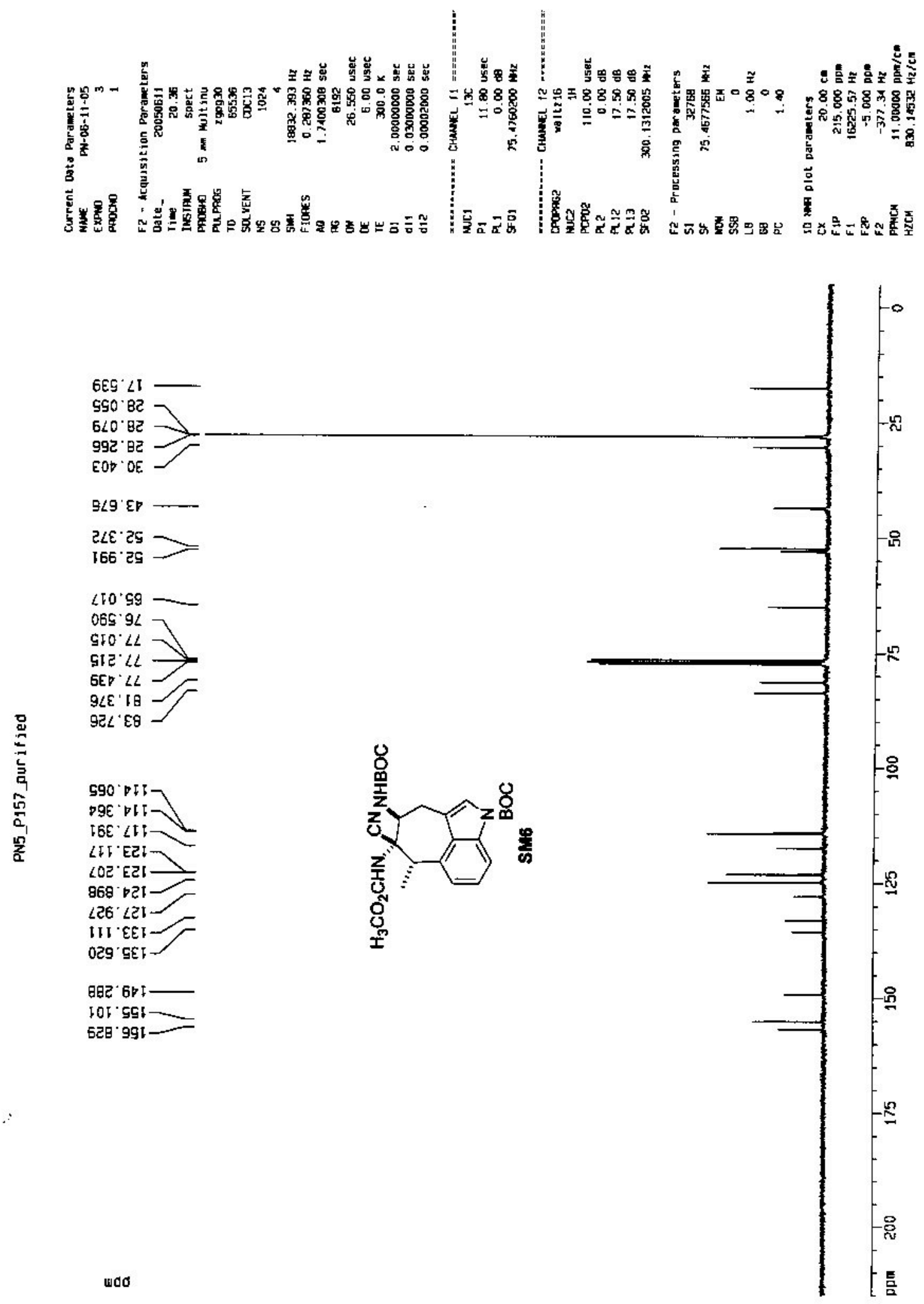

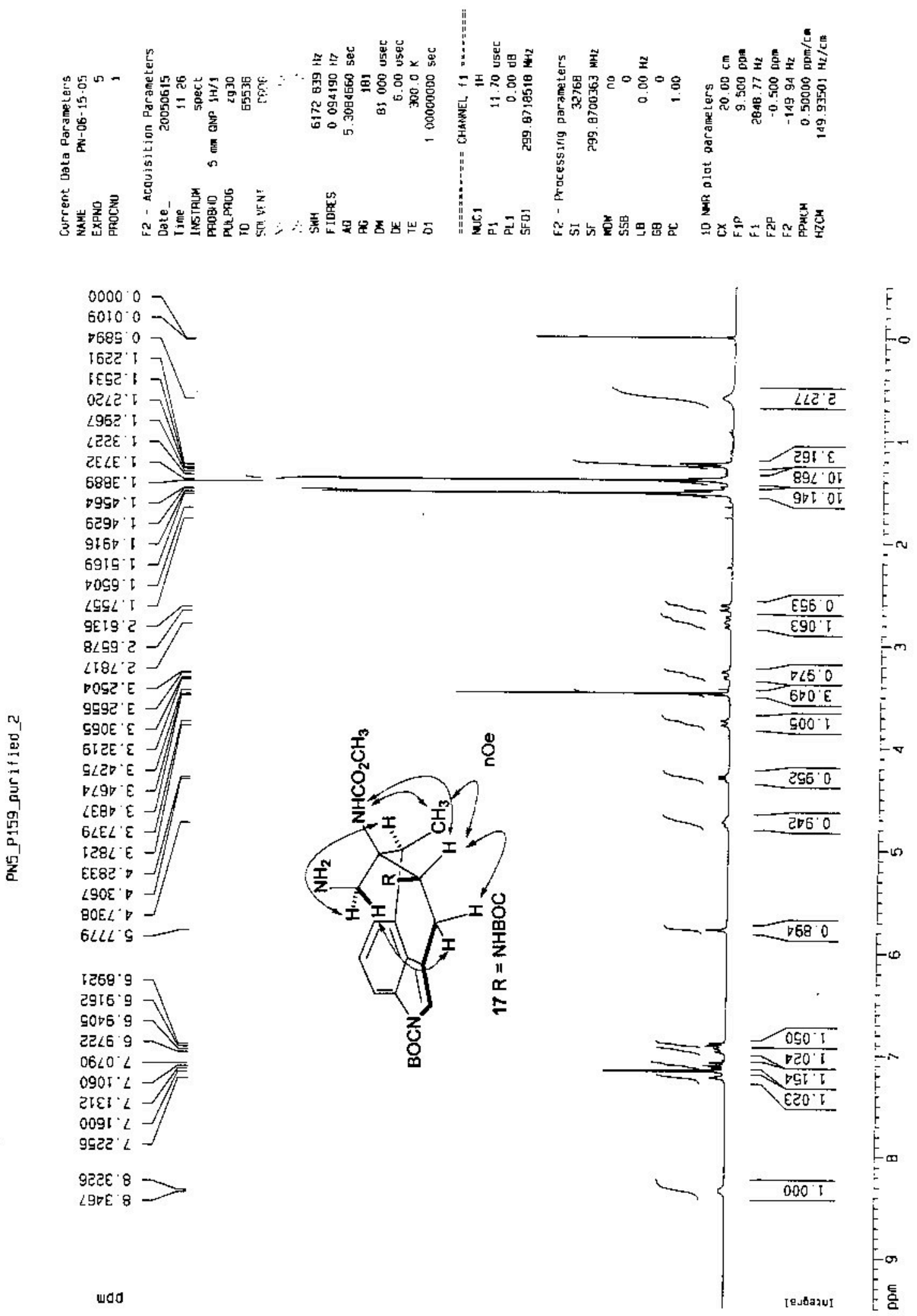

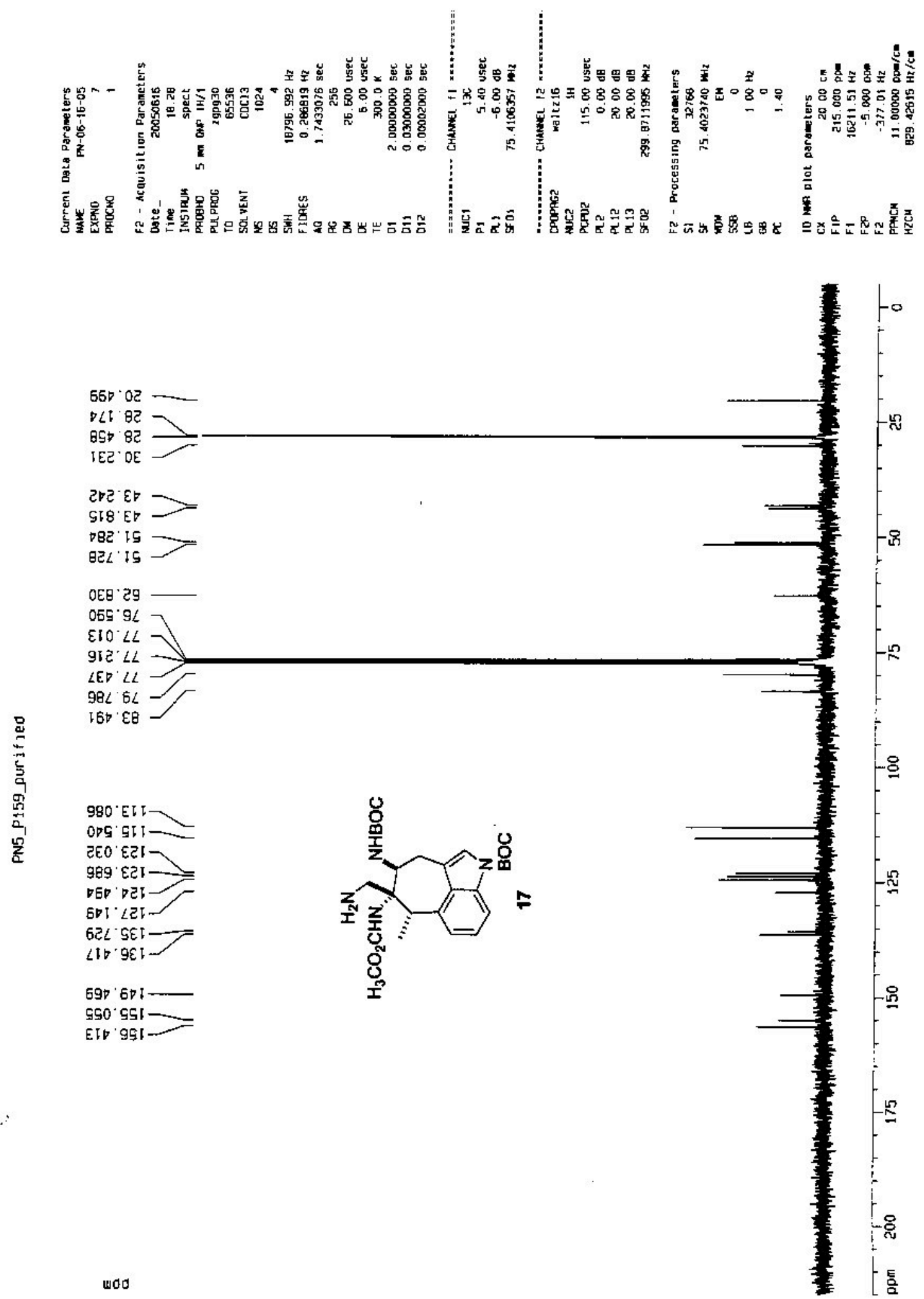

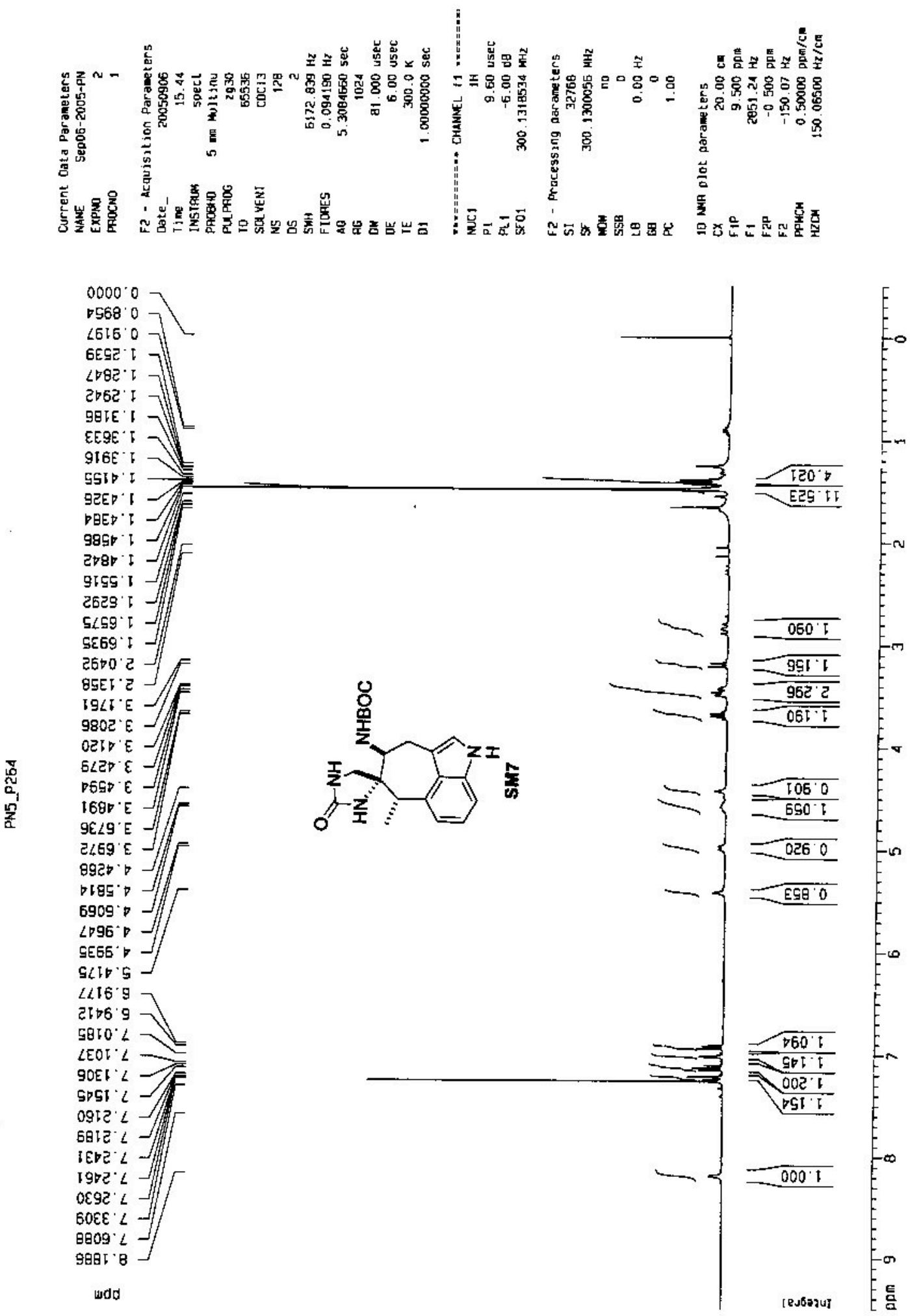


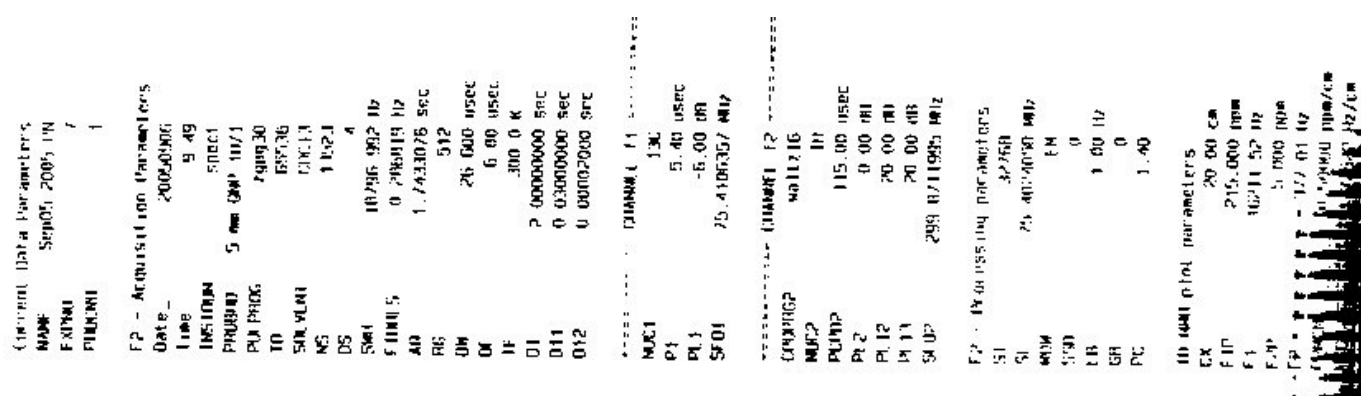

Eis

c:s as

रहड

CLE SC

इอง $3 E$

YPE $9 E$

EEE GE

¿OE

IEL'AE -

390 or -

$\therefore \div=00-1 /$

Eso:0

उटg $จ$

का $50-/$

कas. की -

519 99

Ede $t L$
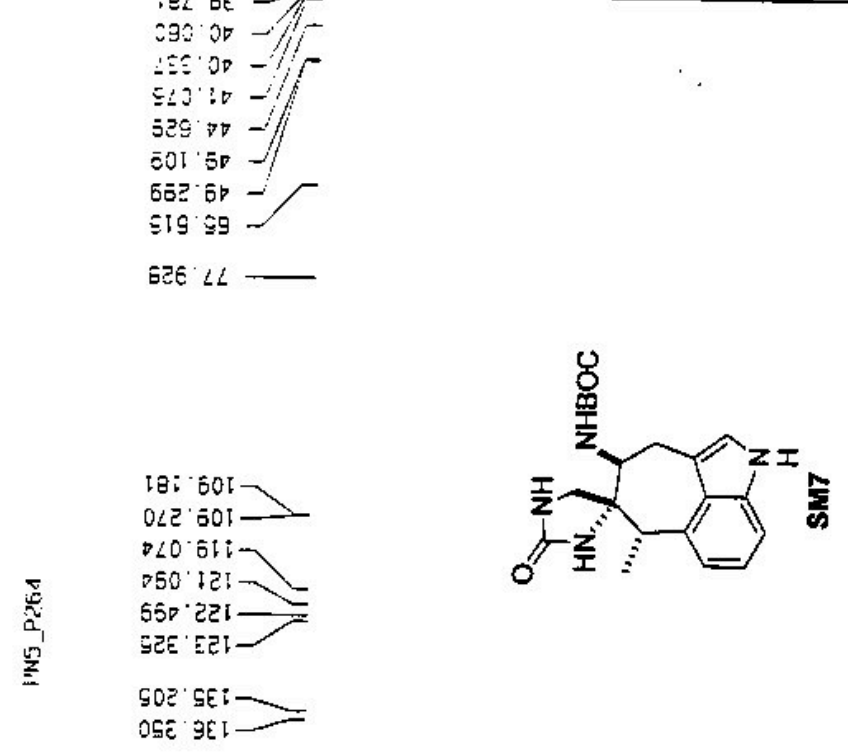

CEE' SST -

$\angle 29 \cdot 191-$
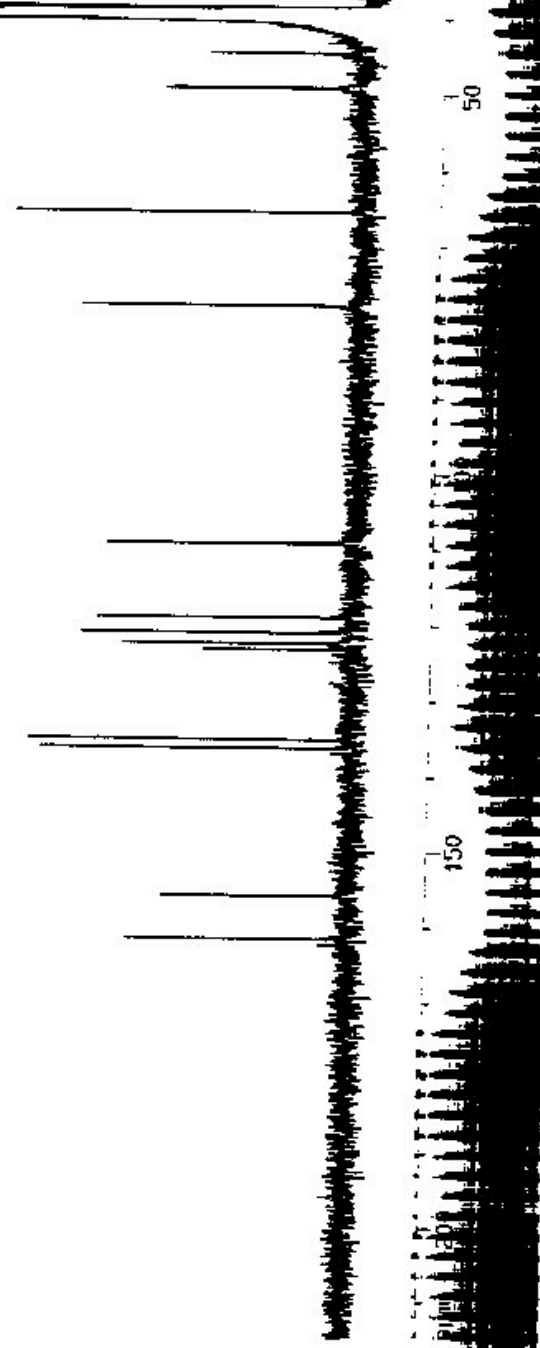

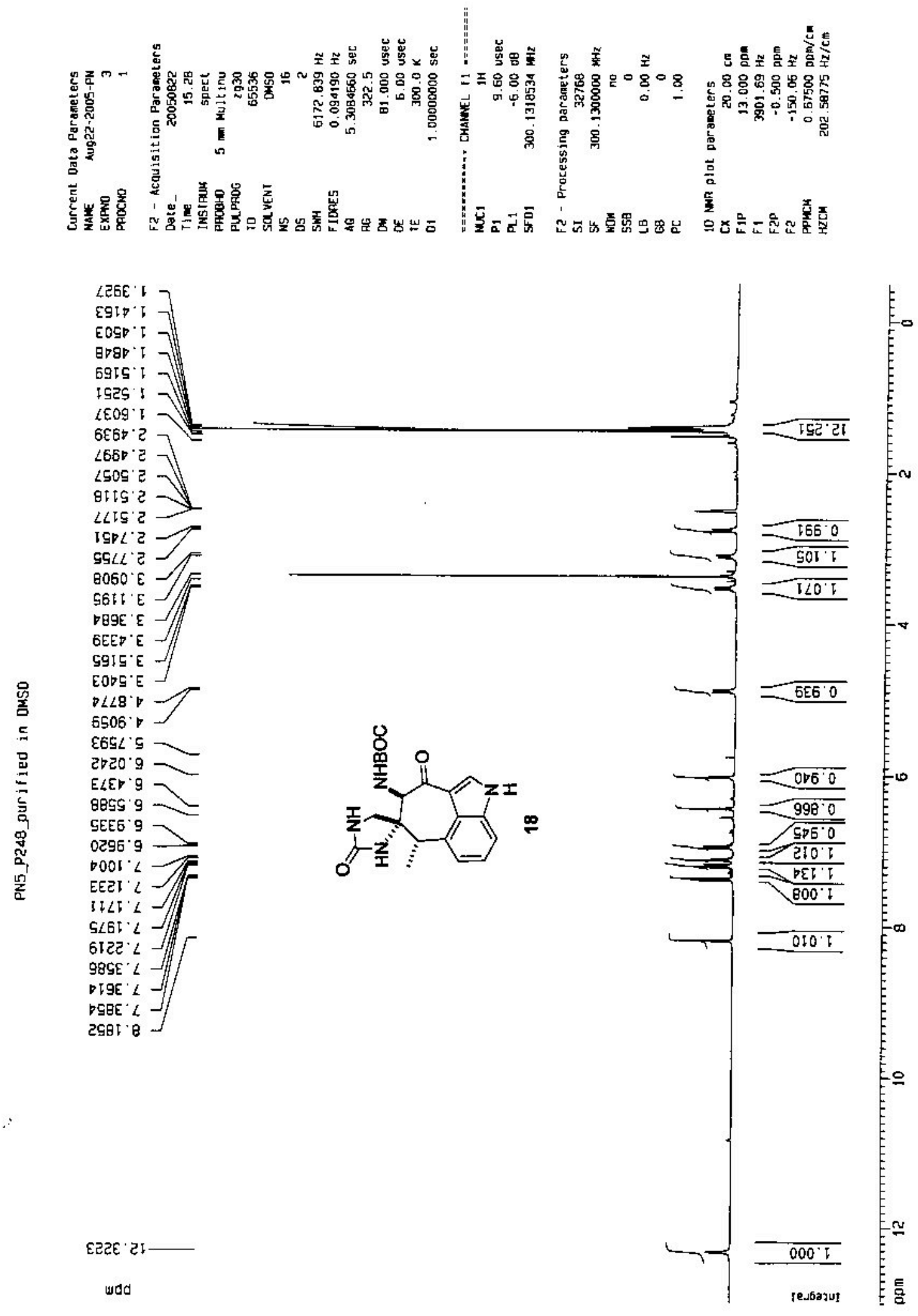

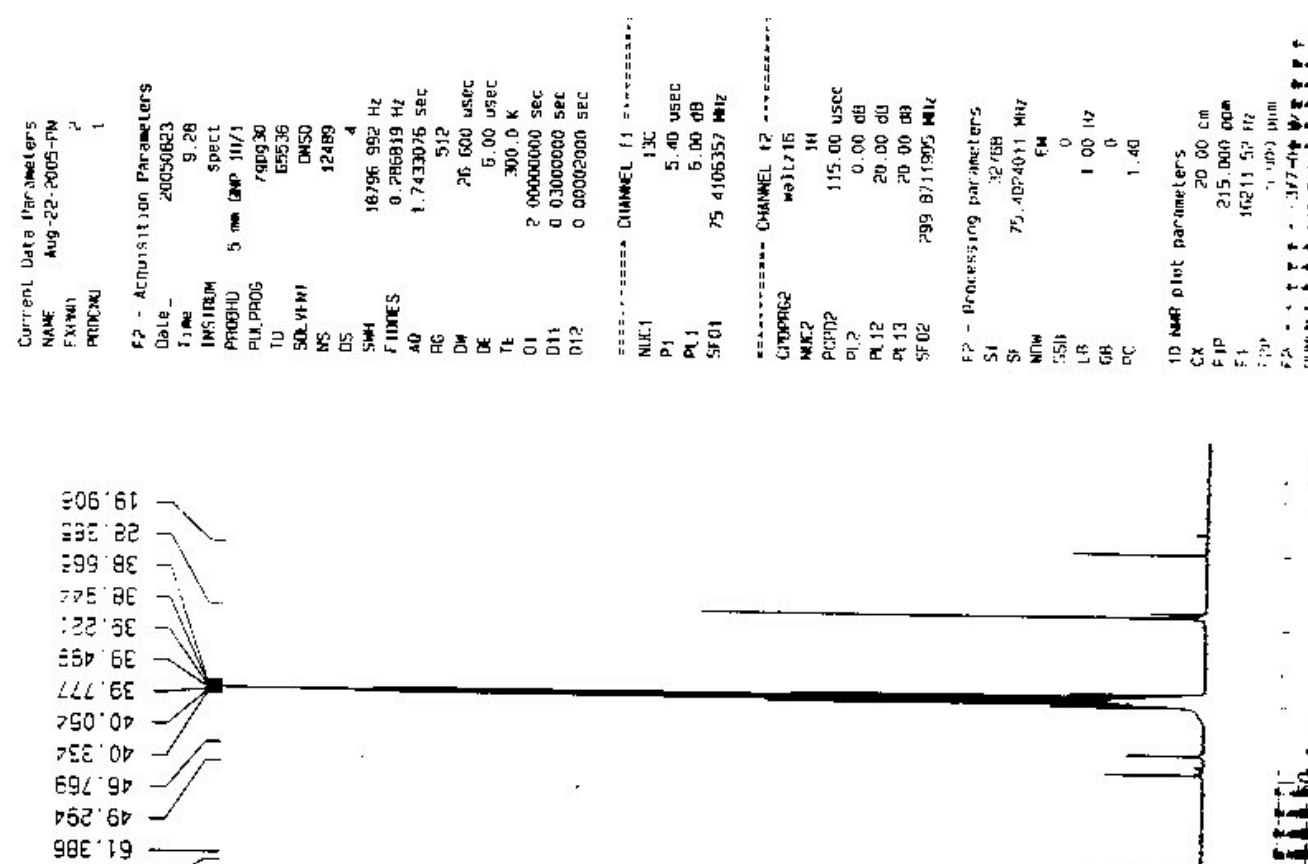

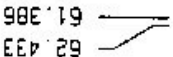

6It $8 L-$

$\frac{0}{0}$
$\frac{0}{5}$
$\frac{5}{2}$
$\frac{5}{2}$
$\frac{2}{2}$
$\frac{2}{2}$

DS9 011-

5eट DII-

ese 25

aes.c5t

$\varepsilon 己 2-\varepsilon \varepsilon 1$

ELQ $\triangle \mathrm{DEI}=$

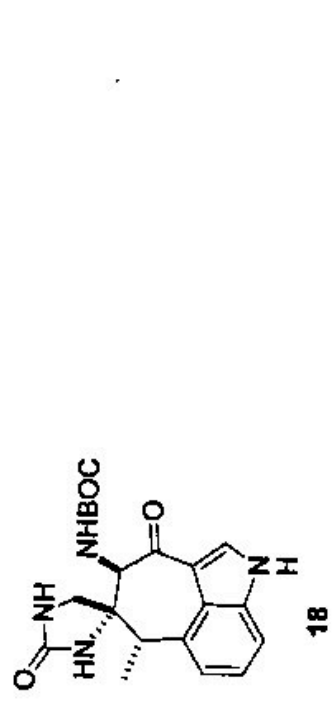

$ट \varepsilon L 9 \varepsilon I-$

898.921

IES.tgi -

$O Z L B B I-$

wad

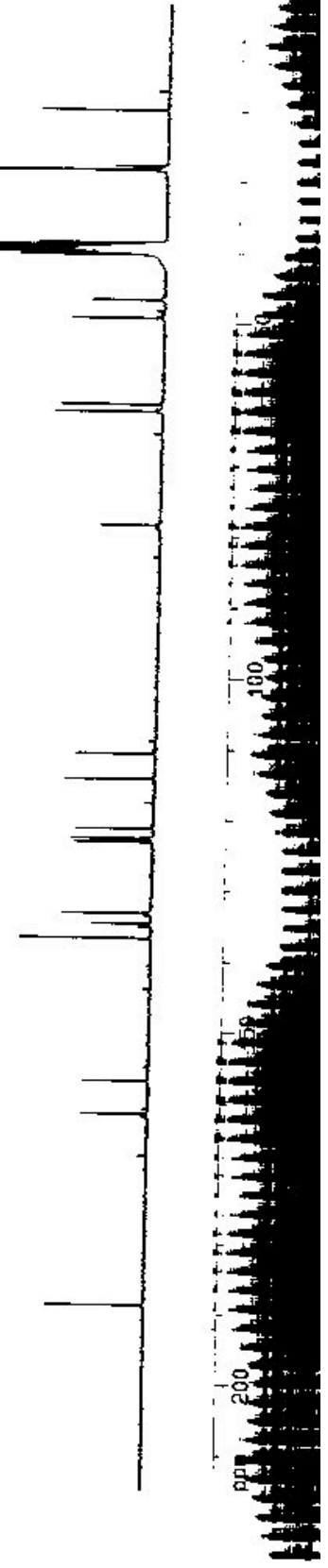


Macromodel 9.0 calculations (MMFF parameter set)

10000-step directed Monte Carlo search about all rotatable bonds. Each minima shown was found over 450 times.

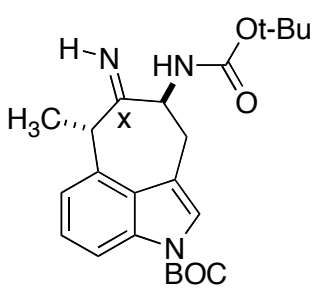

$0.0 \mathrm{Kcal} / \mathrm{mol}$

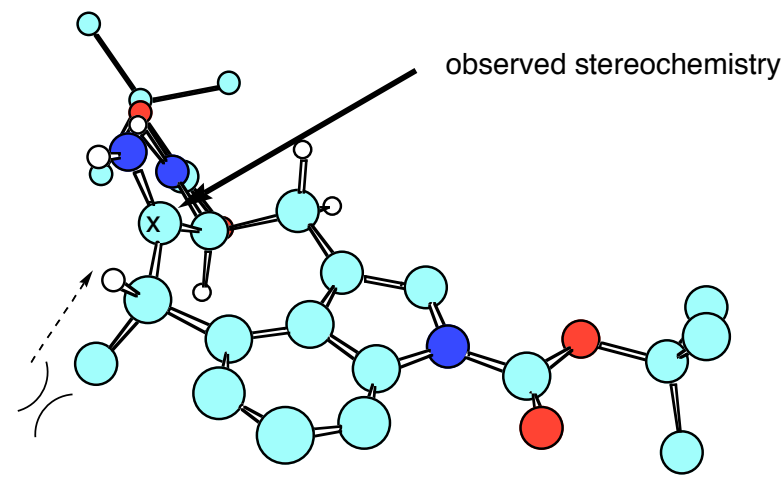

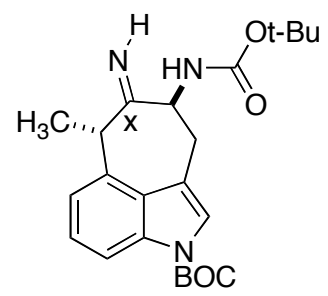

$2.8 \mathrm{Kcal} / \mathrm{mol}$

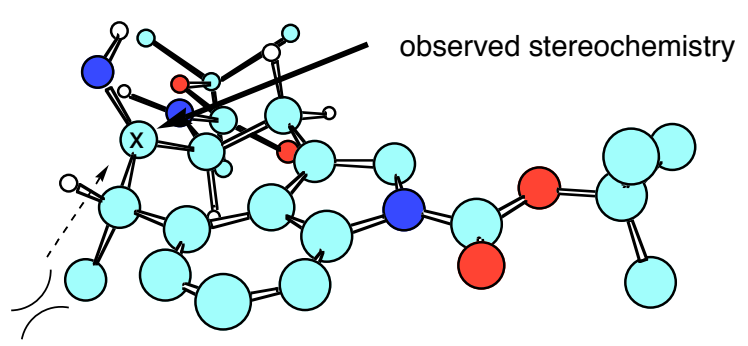<smiles>[Z]C(C)c1cccc2[nH]cc(CC(C)NC(=O)OC(C)=O)c12</smiles>

$3.0 \mathrm{Kcal} / \mathrm{mol}$

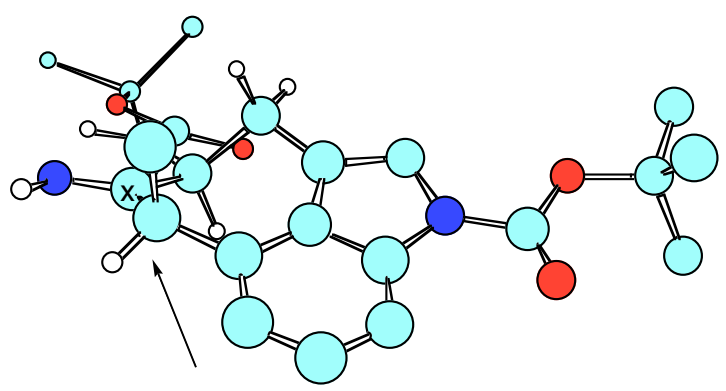<smiles>[R16]c1cccc(C(C)C)c1-c1c[nH]c2cccc([R16])c12</smiles>

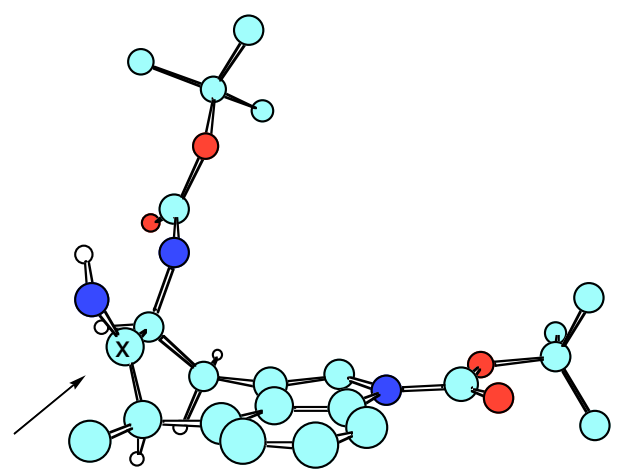

$3.6 \mathrm{Kcal} / \mathrm{mol}$ 